\title{
DENSITY OF SEA WATER
}

AT TIDE STATIONS

\author{
ATLANTIC COAST \\ NORTH AND SOUTH AMERICA
}

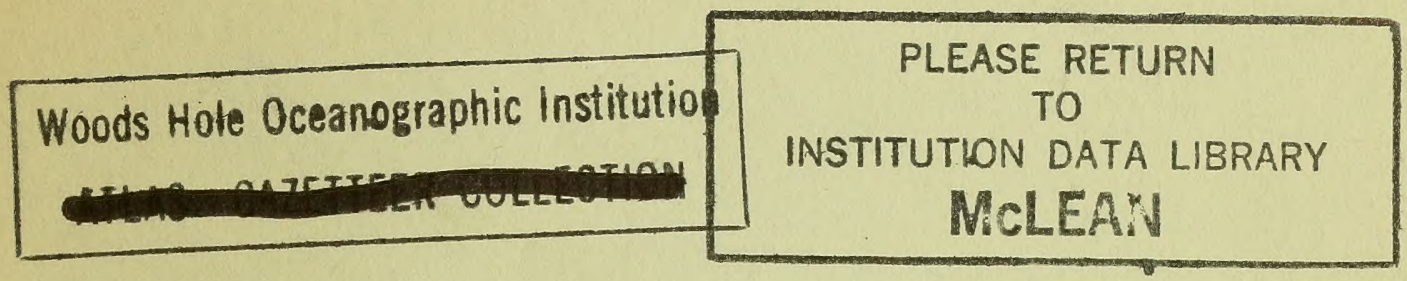

SPECIAL PUBLICATION NO. 279

(FORMERLY NO. DW-1)

REVISED (1953) EDITION

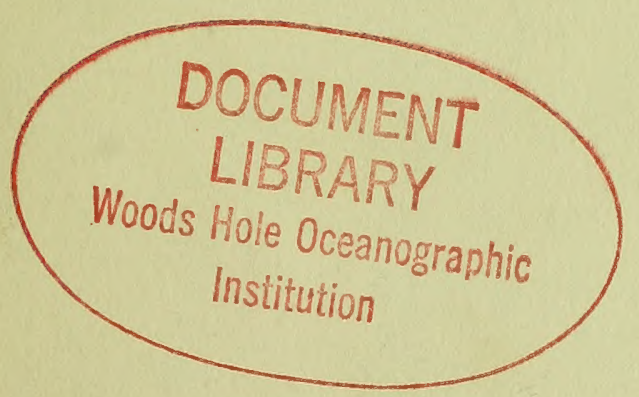

U. S. DEPARTMENT OF COMMERCE COAST AND GEODETIC SURVEY

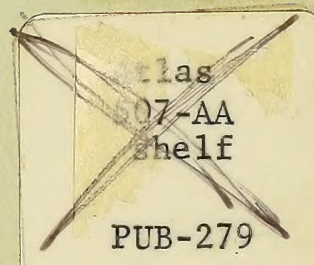





\section{U. S. DEPARTMENT OF COMMERCE \\ SINCLAIR WEEKS, Secretary COAST AND GEODETIC SURVEY \\ ROBERT F. A. STUDDS, Director}

SPECIAL PUBLICATION NO. 279

(FORMERLY NO. DW-1)

REVISED (1953) EDITION

\section{DENSITY OF SEA WATER}

AT TIDE STATIONS

ATLANTIC COAST

NORTH AND SOUTH AMERICA
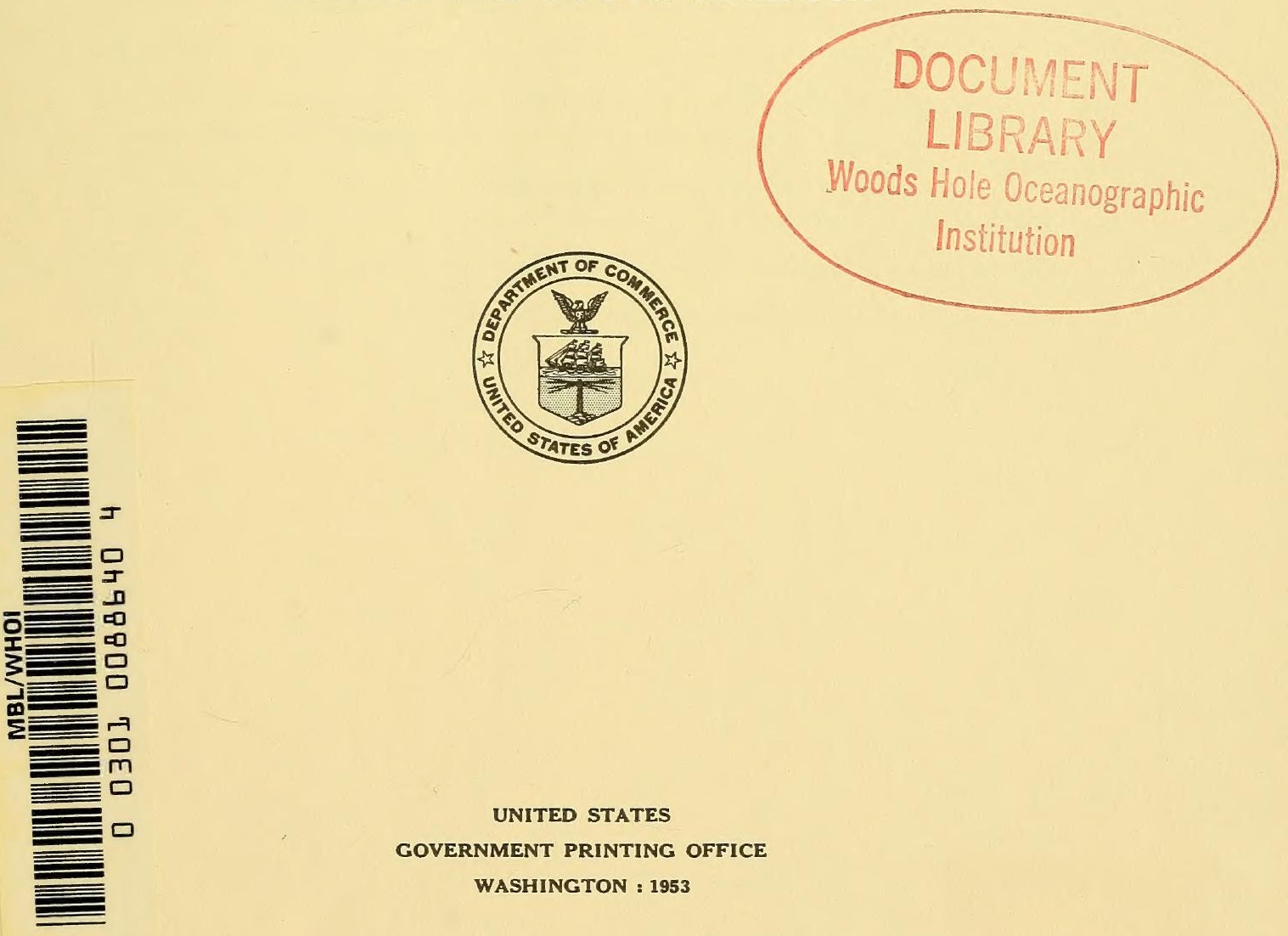

UNITED STATES

GOVERNMENT PRINTING OFFICE

WASHINGTON : 1953 



\section{CONTENTS}

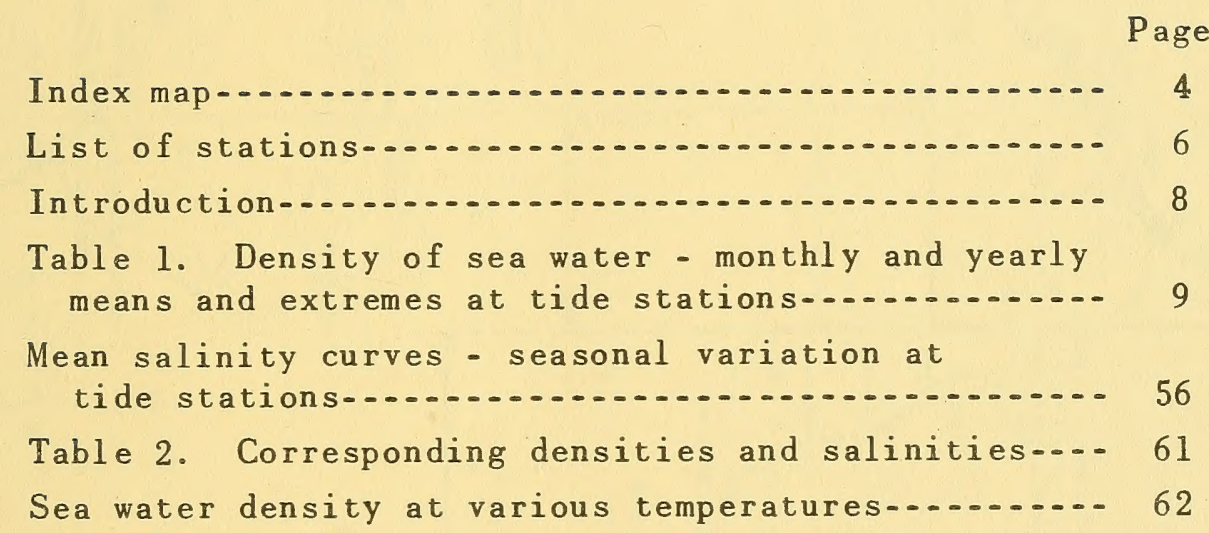




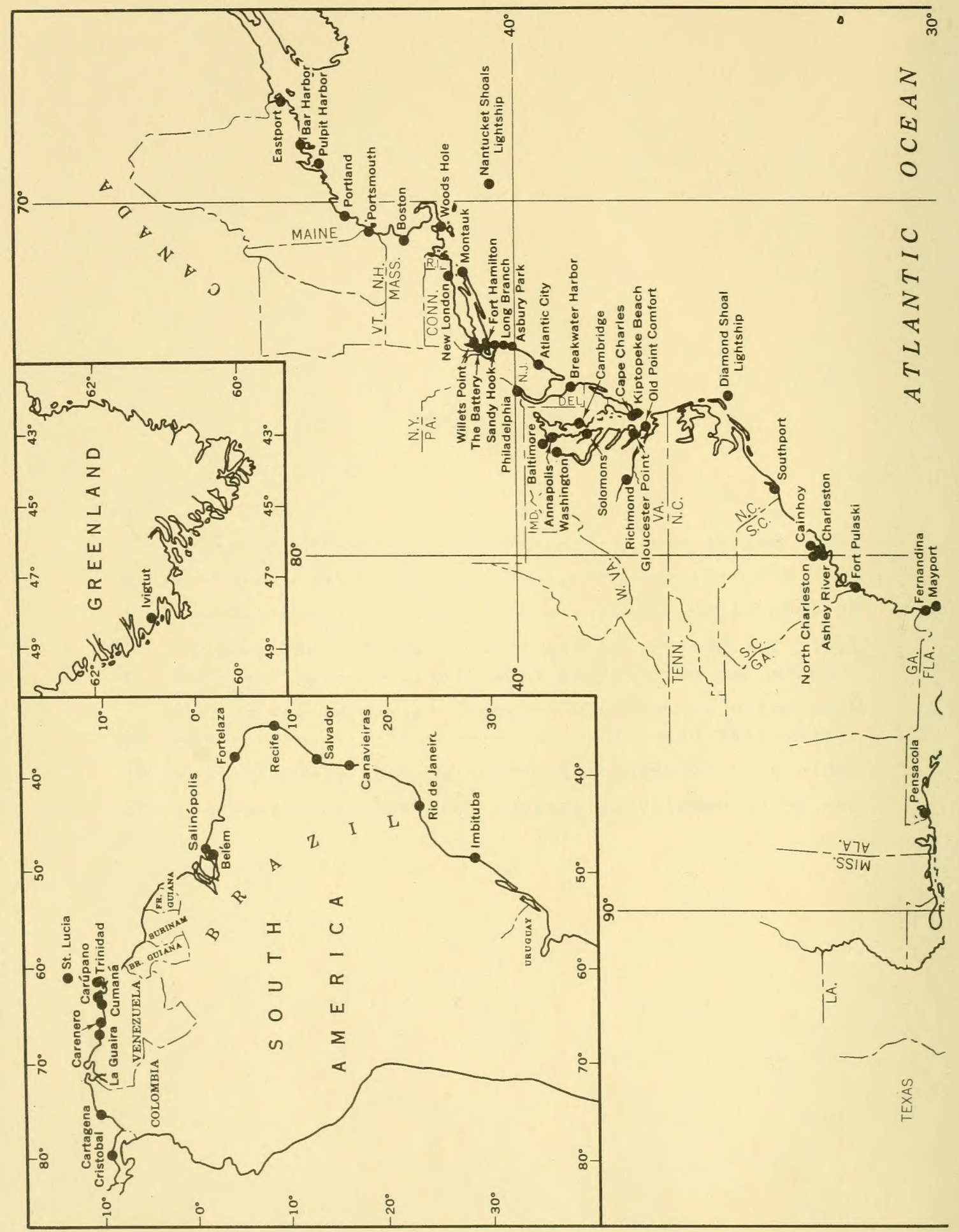




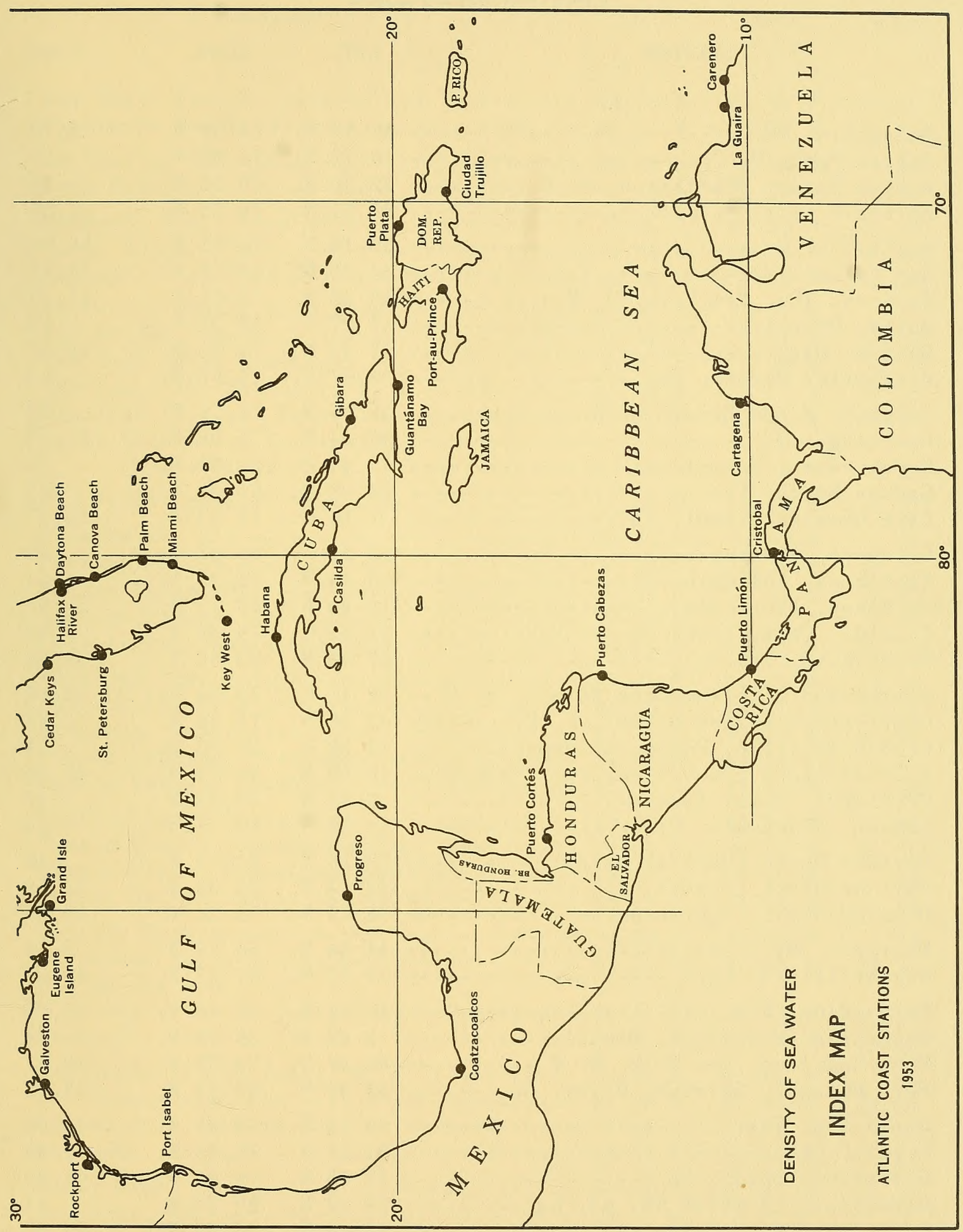




\section{LIST OF STATIONS}

STATION

LAT.

LONG.

PAGE

1

01

Annapolis, Md............................. 3859 N. 7629 W.

28,58

Asbury Park, N. J........................... $4013 \mathrm{~N} .7400 \mathrm{~W}$.

Ashley River, Charleston, S. C....... 3250 N. 7958 W.

33

Atlantic City, N. J......................... 3921 N. 7425 W.

21,57

Baltimore, Md.

$3916 \mathrm{~N} .7635 \mathrm{~W}$.

29,58

Bar Harbor, Me.......................... 4423 N.

$6812 \mathrm{~W}$.

10,56

Battery, The, New York, N. Y.......... $4042 \mathrm{~N}$

$7401 \mathrm{~W}$.

17,57

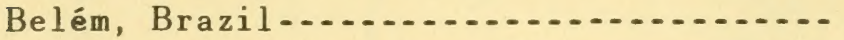

Boston, Mass.

127 S. 4830 W.

Breakwater Harbor, Del ................

$4221 \mathrm{~N}$.

$7103 \mathrm{~W}$.

13,56

$7506 \mathrm{~W}$.

Cainhoy, Wando River, S. C....................

$3255 \mathrm{~N} .7950 \mathrm{~W}$.

33

Cambridge, Md.

$7604 \mathrm{~W}$.

Canavieiras, Brazil.......................... 1541 S. 3859 W.

27,58

55

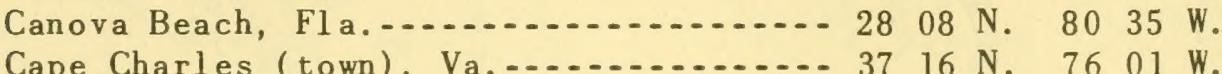

36

24,57

Carenero, Venezuela........................... 1032 N. 6607 W.

7533 W.

51

6315 W.

53

Carúpano, Venezuel a........................ $1040 \mathrm{~N}$.

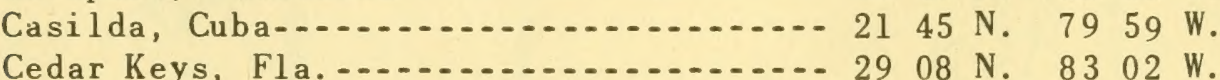

49

39,59

Charleston (Customhouse Wharf), S. C. - 3247 N. 7955 W.

Charleston (Ashley River), S. C....... 3250 N. 7958 W.

$31,32,58$

Ciudad Trujillo, Dominican Republic....- $1828 \mathrm{~N}$.

$6953 \mathrm{~W}$.

Coatzacoalcos (Puerto Mexico), Mexico-.- 1809 N. 9425 W.

Cristobal, Canal Zone-................... $921 \mathrm{~N}$.

$7955 \mathrm{~W}$.

Cumaná, Venezuela............................... 1028 N. 6411 W.

Daytona Beach (Halifax River), Fla..... 2913 N. 8101 W.

Daytona Beach (ocean), Fla..............29 14 N. 8100 W.

Diamond Shoal Lightship_.................. 3505 N. 7520 W.

35,59

30,58

Eastport, Me....................... 4454 N. 6659 W.

Eugene Island, La............................. 2922 N. 9123 W.

9,56

41,59

Fernandina, Fla............................. 3041 N. 8128 W.

Fortelaza (Mucuripe), Brazil-............ 342 S. 3829 W.

34,58

54

Fort Hamilton, New York, N. Y.-...... 4037 N. 7402 W.

Fort Pulaski, Savannah River, Ga...... 3202 N. 8054 W.

18,57

33,58

Galveston, Tex............................... 2919 N. 9448 W.

Gibara, Cuba-................................. 2107 N. 7607 W.

42,60

48

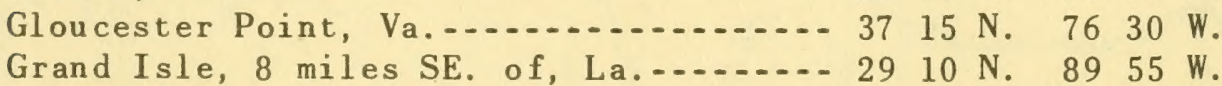

Guantánamo Bay, Cuba-........................ 1954 N. 7509 W.

Habana, Cuba................................. 2309 N. 820 W.

Halifax River, Daytona Beach, Fla.....29 13 N. 8101 W.

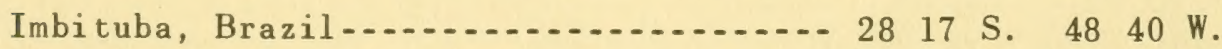

Ivigtut, Greenland 
PAGE

Key West, Fla.

Long Branch, N. J. .................. $4.018 \mathrm{~N}$.

$6656 \mathrm{~W}$.

Mayport, Fla......................... $3024 \mathrm{~N}$.

$8126 \mathrm{~W}$.

35,59

Miami Beach, Fla..................... $2546 \mathrm{~N}$. $8008 \mathrm{~W}$.

Montauk, Fort Pond Bay, L. I., N. Y....- 4103 N.

7158 W. 15,57

Nantucket Shoals Lightship............ $4037 \mathrm{~N}$.

$6937 \mathrm{~W}$.

New London, Conn......................... $4122 \mathrm{~N}$.

$7206 \mathrm{~W}$.

15,56

New York (Fort Hamilton), N. Y......... $4037 \mathrm{~N}$.

$7402 \mathrm{~W}$

18,57

New York (The Battery), N. Y......... $4042 \mathrm{~N}$.

$7401 \mathrm{~W}$.

17,57

New York (Willets Point), N. Y......... 404.8 N.

North Charleston Terminals,

7347 W. 16,57

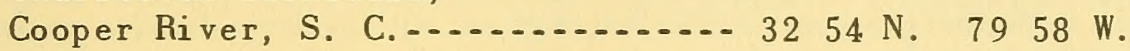

Old Point Comfort, Va............... 3700 N.

$7618 \mathrm{~W}$.

25,57

Palm Beach, Fla...................... $2643 \mathrm{~N}$.

$8002 \mathrm{~W}$.

Pensacola, Fla.................................... $3024 \mathrm{~N}$

$8713 \mathrm{~W}$.

40,59

Philadelphia, $\mathrm{Pa}$.

$3957 \mathrm{~N}$.

75. $08 \mathrm{~W}$.

23,57

Port-au-Prince, Haiti-................. $1833 \mathrm{~N}$.

$7221 \mathrm{~W}$.

49

Port Isabel, Tex..................... 2604 N.

$9713 \mathrm{~W}$.

Portl and, Me.

$4340 \mathrm{~N}$.

Portsmouth, N. H. .................. 4305 N.

$7015 \mathrm{~W}$.

$7045 \mathrm{~W}$.

44,60

11,56

Progreso, Mexico

$2117 \mathrm{~N}$.

$8940 \mathrm{~W}$.

12,56

Puerto Cabezas, Nicaragua...................... $1401 \mathrm{~N}$.

$8323 \mathrm{~W}$.

45,60

Puerto Cortés, Honduras............ 1550 N.

$8757 \mathrm{~W}$.

46

46

Puerto Limón, Costa Rica-.................. 1000 N.

$8302 \mathrm{~W}$.

Puerto Mexico (Coatzacoalcos), Mexico-... $1809 \mathrm{~N}$.

$9425 \mathrm{~W}$.

Puerto Plata, Dominican Republic-...... 1949 N.

$7042 \mathrm{~W}$.

46

45,60

$6853 \mathrm{~W}$.

50

Pulpit Harbor, Me................... $4409 \mathrm{~N}$

3452 W.

11

Recife, Brazil..................... 803 S.

$7725 \mathrm{~W}$.

54

Ri chmond, Va.

$3732 \mathrm{~N}$.

Rio de Janei ro

(Fortaleza de Santa Cruz), Brazil-..- 2256 S.

$4308 \mathrm{~W}$. $9703 \mathrm{~W}$.

25,57

Rockport, Tex................................. $2801 \mathrm{~N}$.

$6058 \mathrm{~W}$.

St. Lucia (Vieux Fort), B. W. I....... 1344 N.

8238 W.

43,60

St. Petersburg, Fla. $2746 \mathrm{~N}$

$4723 \mathrm{~W}$.

Salinópolis, Brazil_.................. $037 \mathrm{~S}$.

$3831 \mathrm{~W}$.

38,59

54

Salvador, Brazil_.................... 1258 S

$7401 \mathrm{~W}$.

Sandy Hook, N. J....................... 4028 N.

$7627 \mathrm{~W}$. 19,57

Solomons, Md................................... 3819 N.

$7801 \mathrm{~W}$.

27,57

Sou thport, N. C. ..................... 3355 N.

$6136 \mathrm{~W}$.

31,58

Trinidad (Carenage Bay), B. W. I...... $1041 \mathrm{~N}$

Washington, D. C. ................... $3852 \mathrm{~N}$.

$7701 \mathrm{~W}$. 2657

Willets Point, New York, N. Y........ $4048 \mathrm{~N}$.

$7347 \mathrm{~W}$.

$7040 \mathrm{~W}$.

16,57 14,56 


\section{INTRODUCTION}

The summaries of sea water densities presented in this publication are based on observations made in Atlantic harbor and coastal waters through the year 1952. The densities were observed primarily at tide stations which, in the United States, were maintained by the Coast and Geodetic Survey, often with the cooperation of other organizations. Final results for Venezuela were supplied by the Ministerio de-Obras Publicas, Estados Unidos de Venezuela. For other countries the Coast and Geodetic Survey derived the data from observations made by organizations in the countries concerned. In Latin America the observations were obtained through the cooperation of the Inter American Geodetic Survey.

Table 1 presents monthly means and annual mean and extremes for each year of observations. Maximum and minimum densities are shown for each year in which observations were made, whether or not the observations covered the whole year. In all cases the yearly extremes are from the months of the year for which means are given. If they are from an incomplete year, they are followed by an asterisk.

For each station at which the series of observations covered two or more years, there are given also the following monthly values for the series: the mean of the monthly means together with their corresponding salinities, the maximum density observed, the mean of the monthly maxima, the mean of the monthly minima, and minimum observed.

Following the table of densities are graphs showing the seasonal variation in salinity at stations for which the observations covered five years or more.

The observations are made by drawing a sample of water from near the surface and observing its density by means of a hydrometer. They are usually made once each weekday at whatever time the observer attends the tide gage. It may be assumed that in the course of a month or a year the distribution of observations is fairly uniform over all phases of the tide. At some stations situated within or near the entrance of an estuary, the density varies with the stage of the tide or with the direction of the tidal current.

The densities in Table 1 are given with respect to the density of pure water at a temperature of $4^{\circ} \mathrm{C}\left(39.2^{\circ} \mathrm{F}\right)$ as unity. The actual density of the water may vary from a little less than unity for fresh water at a temperature other than $4^{\circ} \mathrm{C}$ to approximately 1.0310 for the heaviest sea water. Since the density of sea water, as observed, depends not only upon the amount of soluble matter contained in a unit volume but also upon the temperature of the water at the time the reading was made, it is necessary to reduce the observed densities to some standard temperature in order that they may be comparable. In this publication the observed densities have been reduced to a standard temperature of $15^{\circ} \mathrm{C}\left(59^{\circ} \mathrm{F}\right)$. The density of pure water free from air at a temperature of $15^{\circ} \mathrm{C}$ is 0.9991 .

The salinity of sea water is defined as the number of grams of salts contained in 1000 grams of sea water. Salinity can be determined by several different methods, one of the simplest being based upon the density of the water as obtained from the use of the hydrometer. Table 2 gives the salinity corresponding to different values of density at the standard temperature of $59^{\circ} \mathrm{F}$. $\left(15^{\circ} \mathrm{C}\right.$. ) to which all densities in this publication are referred.

For some practical uses of density data, it is more important to know the density at the temperature apt to be encountered than at the standard temperature. The graph, Sea Water Density at Various Temperatures, provides for converting density at $59^{\circ} \mathrm{F}\left(15^{\circ} \mathrm{C}\right)$ to density at other temperatures.

The following publications complete this series:

Special Pub. 278. Surface Water Temperatures, Atlantic Coast, North and South America.

Special Pub. 280. Surface Water Temperatures, Pacific Coast, North and South America and Pacific Ocean Islands.

Special Pub. 281. Density of Sea Water, Pacific Ocean. 


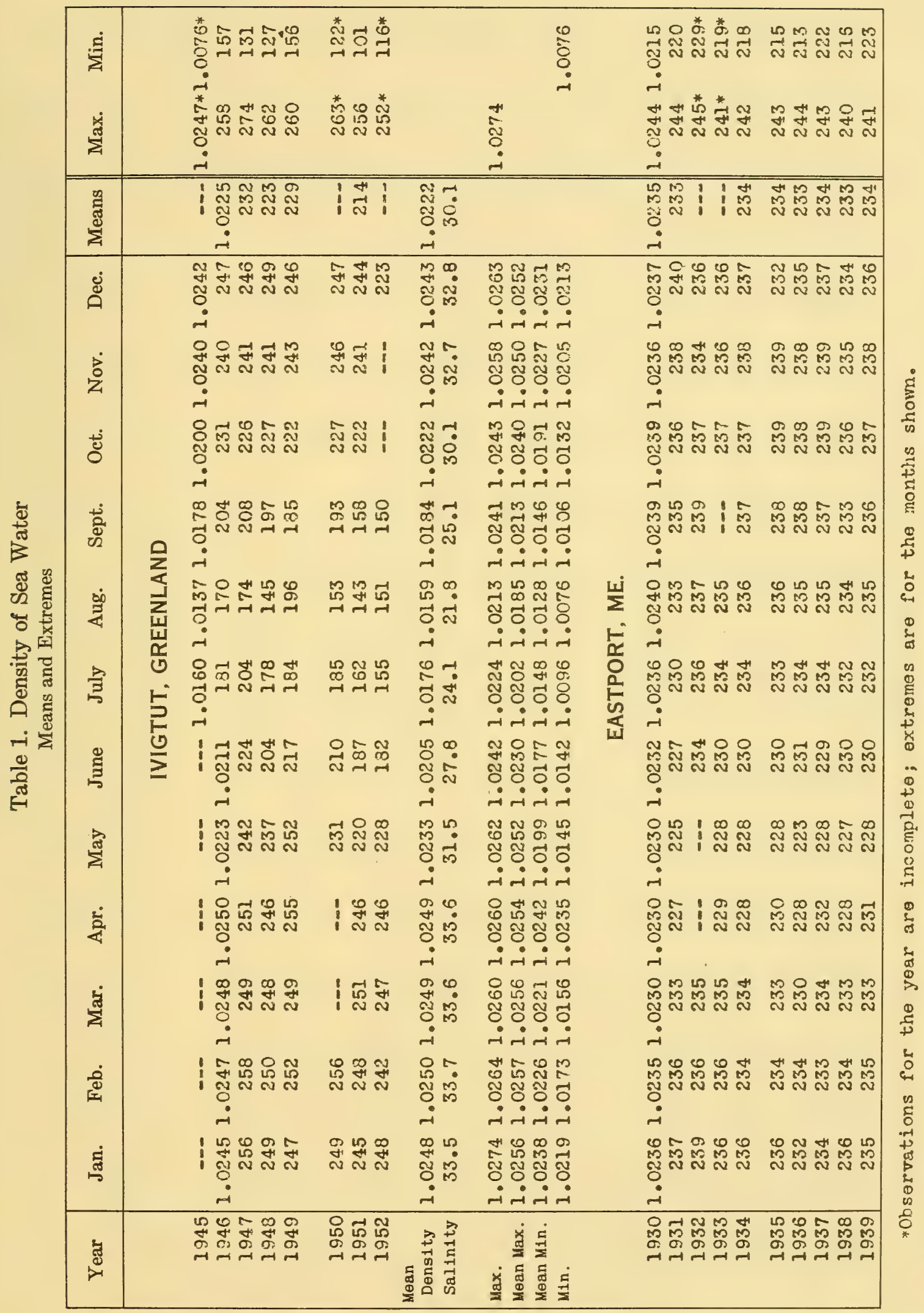




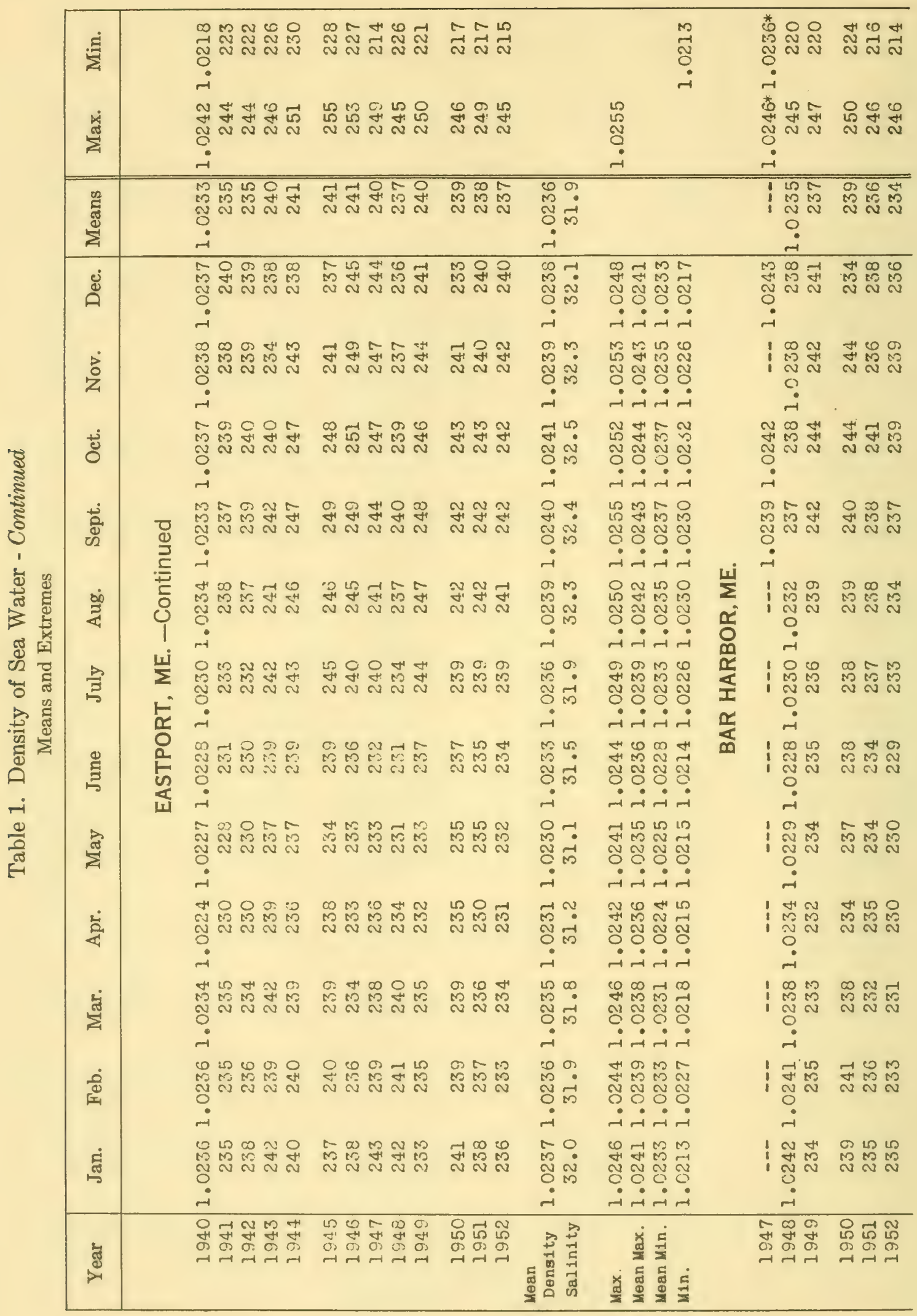




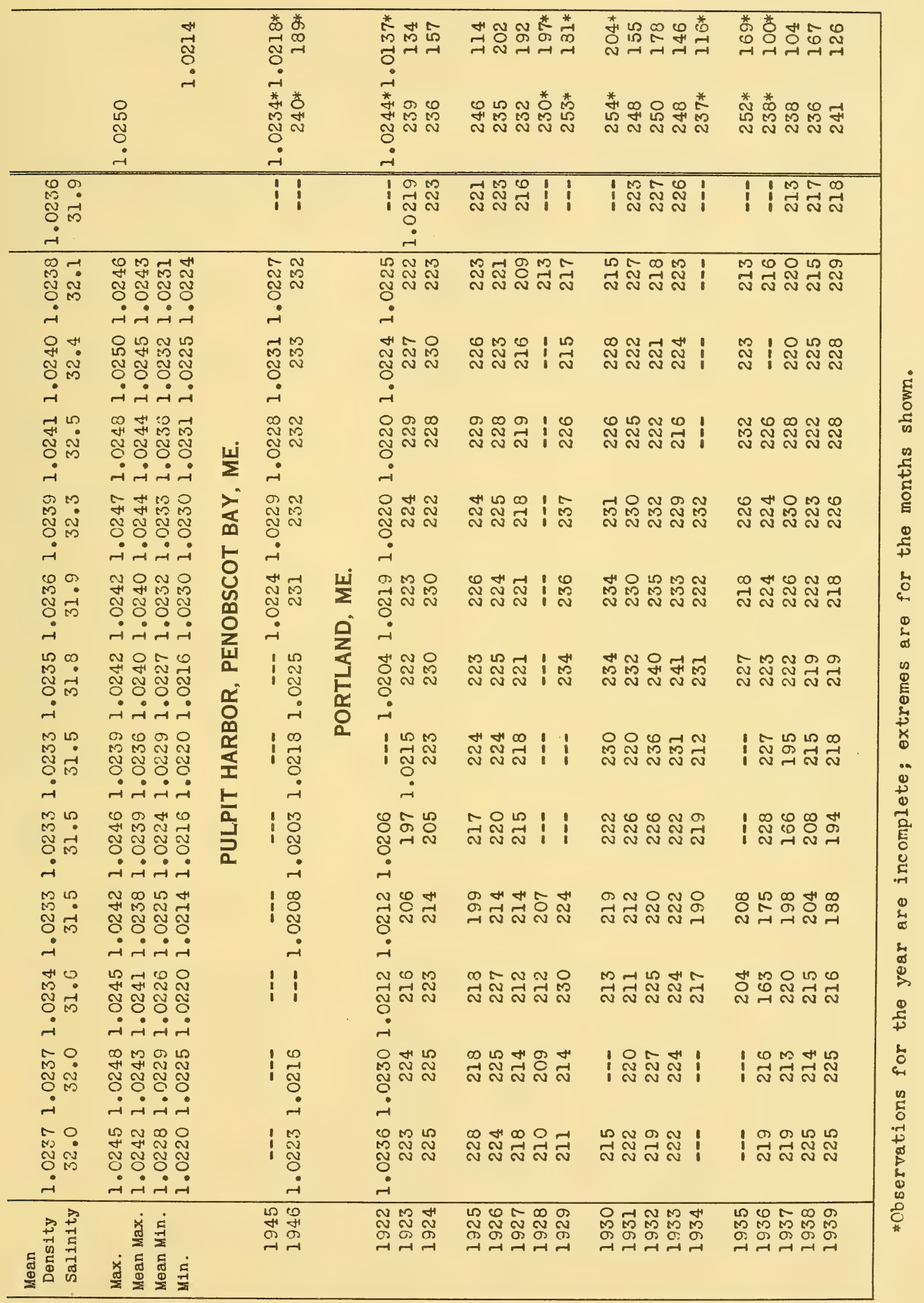




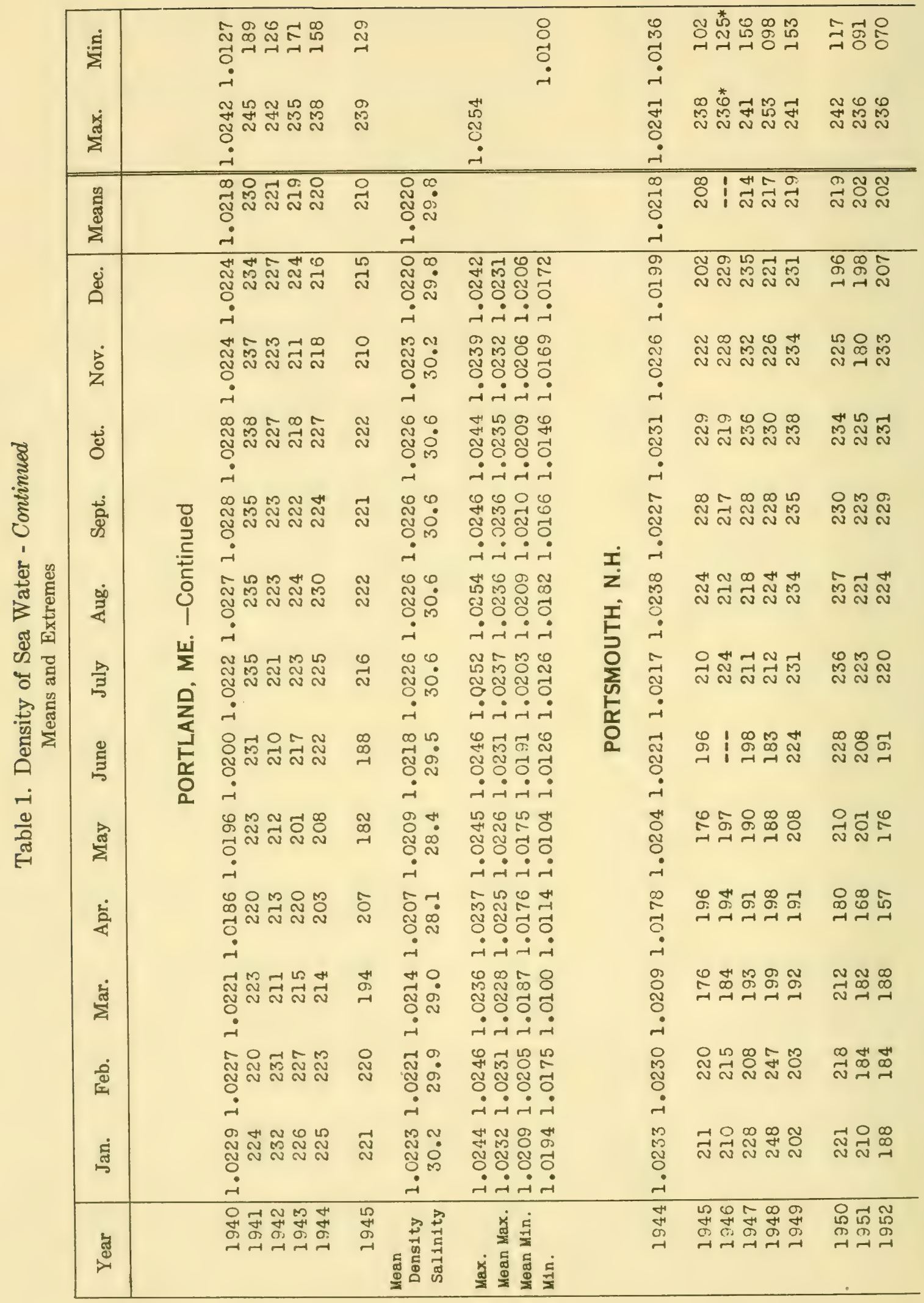




\begin{tabular}{|c|c|c|c|c|c|c|c|}
\hline & $\begin{array}{l}0 \\
8 \\
8 \\
0 \\
-1\end{array}$ & & 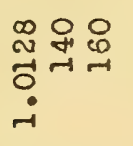 & 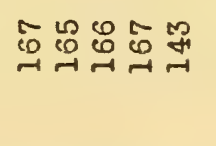 & 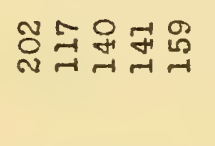 & Mี & 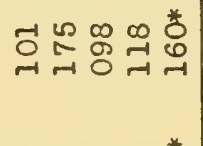 \\
\hline & 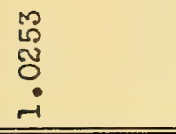 & & 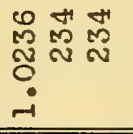 & 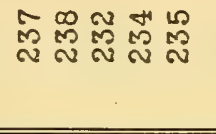 & 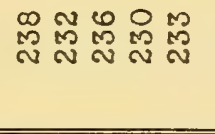 & స్ల & 品罢占心 \\
\hline 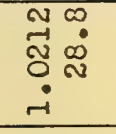 & & & 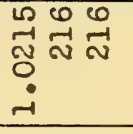 & ज્ञ & 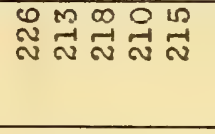 & 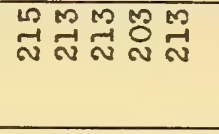 & 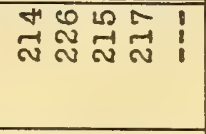 \\
\hline 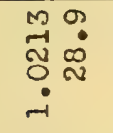 & 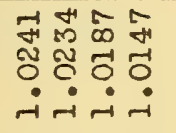 & & 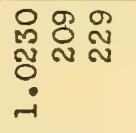 & 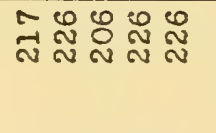 & స్心 & 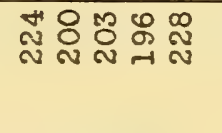 & 尚 \\
\hline $\begin{array}{l}m \text { : } \\
\text { N } \\
0 \\
0 \\
-1\end{array}$ & 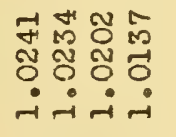 & & 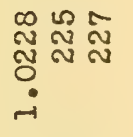 & 斻 & 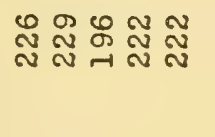 & 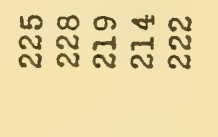 & 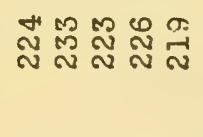 \\
\hline $\begin{array}{l}0 \\
\text { m } \\
0 \\
0\end{array}$ & 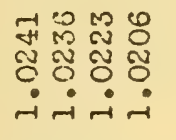 & & 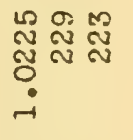 & 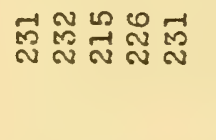 & 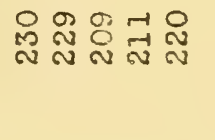 & M心 & 昰心恥心 \\
\hline $\begin{array}{l}5 \\
\text { Nิ } \\
0 \\
0 \\
-1\end{array}$ & 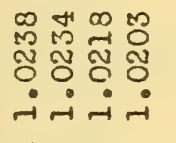 & & 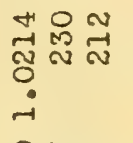 & $\begin{array}{l}\infty \vec{N} 0 \vec{心} \vec{心} \\
\sim \vec{N}\end{array}$ & 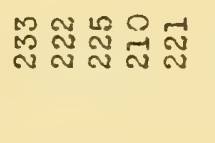 & 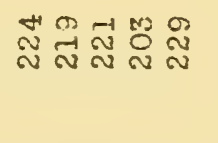 & 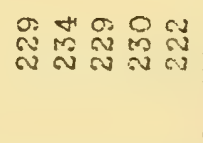 \\
\hline 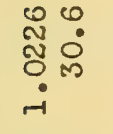 & 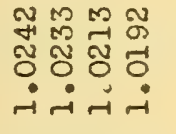 & 这 & 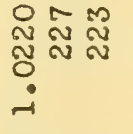 & 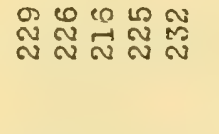 & 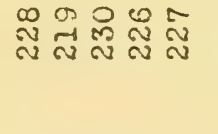 & $\begin{array}{l}\mathrm{N} \\
\mathrm{N} 心 \\
\mathrm{~N}\end{array}$ & 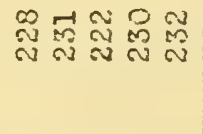 \\
\hline 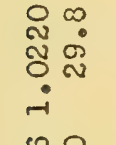 & 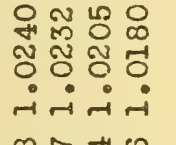 & o & 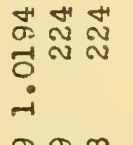 & 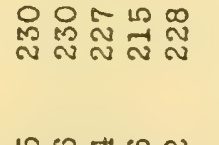 & 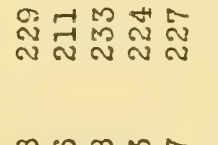 & 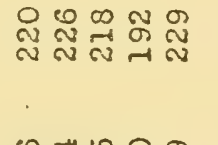 & 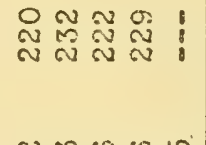 \\
\hline 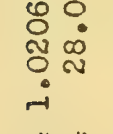 & 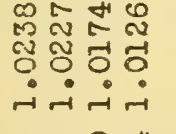 & & $\begin{array}{lll}9 & \infty & \infty \\
\sigma & -1 & -1 \\
-1 & 0 & 0 \\
0 & \end{array}$ & 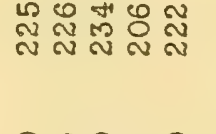 & 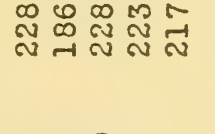 & 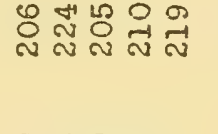 & 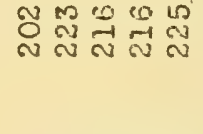 \\
\hline 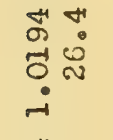 & 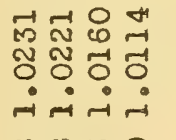 & & 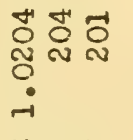 & 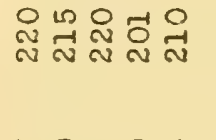 & 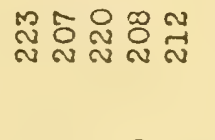 & 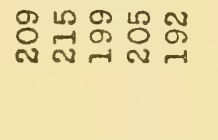 & 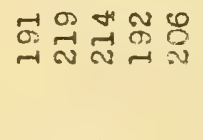 \\
\hline $\begin{array}{l}\text { म1 } \\
\infty \\
-1 \\
0 \\
0 \\
0 \\
-1\end{array}$ & 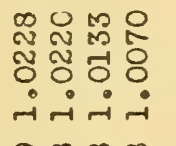 & & 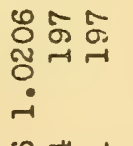 & 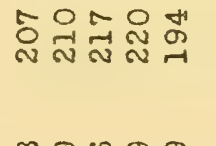 & 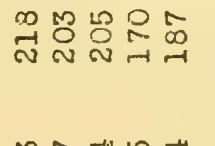 & $\begin{array}{lllll} & 1 & 1 & 0 & 0 \\
\infty & \infty & 0 & 0 \\
-1 & \sim & \sim & \sim\end{array}$ & 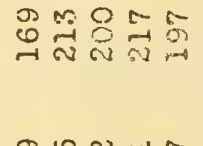 \\
\hline $\begin{array}{ll}m & m \\
\sigma & 0 \\
-1 & 0 \\
0 & 0\end{array}$ & 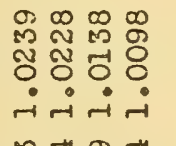 & & 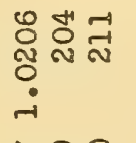 & 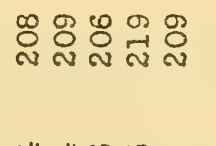 & 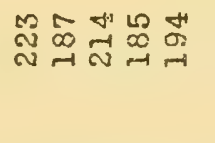 & 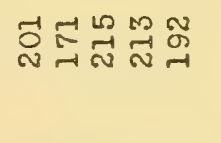 & 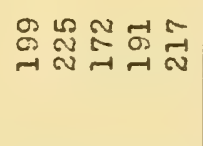 \\
\hline 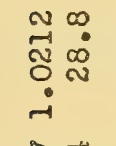 & 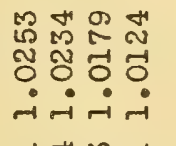 & & 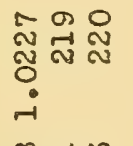 & 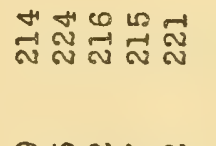 & 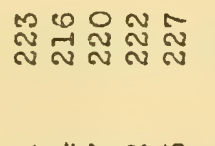 & 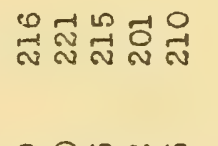 & 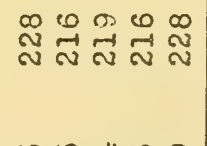 \\
\hline 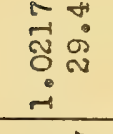 & 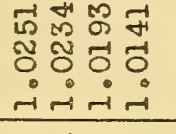 & & 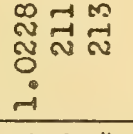 & 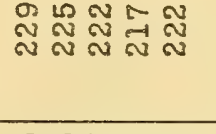 & స్సు స్తి & 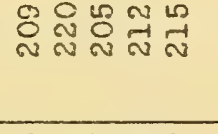 & 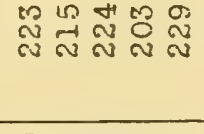 \\
\hline 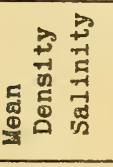 & 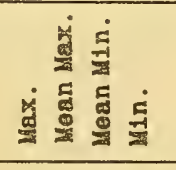 & & 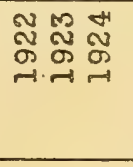 & 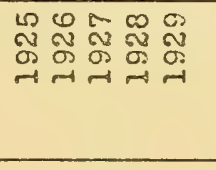 & 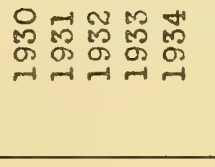 & 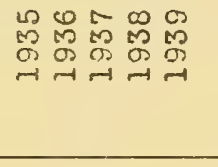 & 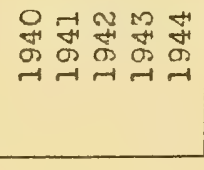 \\
\hline
\end{tabular}




\begin{tabular}{|c|c|c|c|c|c|c|c|c|c|}
\hline 哭 & & 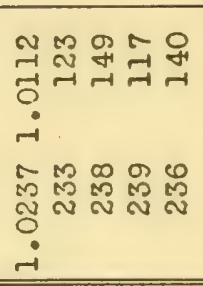 & 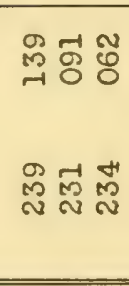 & & 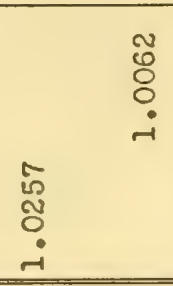 & & 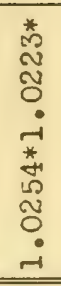 & 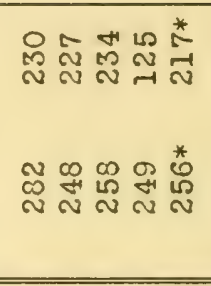 & 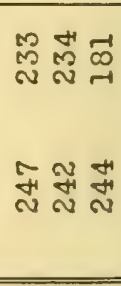 \\
\hline 荧 & & 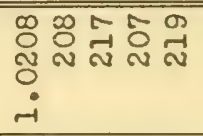 & స్త్రే & 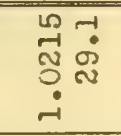 & & & ! & 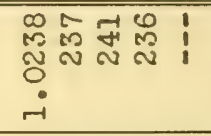 & 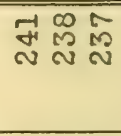 \\
\hline ஓ் & & 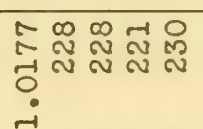 & 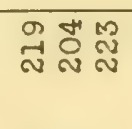 & 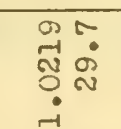 & 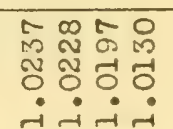 & & 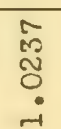 & 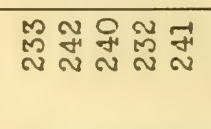 & 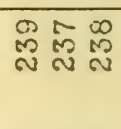 \\
\hline 宫 & & 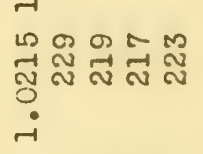 & 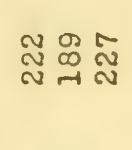 & 芯 & 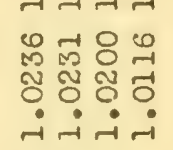 & & $\begin{array}{l}-1 \\
\infty \\
\infty \\
\tilde{N} \\
0 \\
-1\end{array}$ & 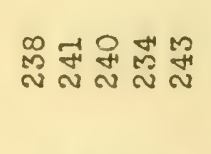 & 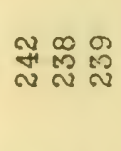 \\
\hline हे & & 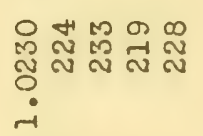 & 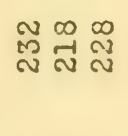 & 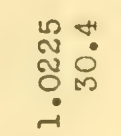 & 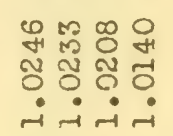 & & $\begin{array}{l}\stackrel{9}{4} \\
\stackrel{\sim}{\tilde{~}} \\
\text { : }\end{array}$ & 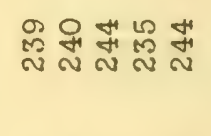 & 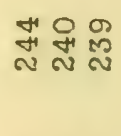 \\
\hline $\begin{array}{l}\text { 茪 } \\
\text { कू. }\end{array}$ & d & 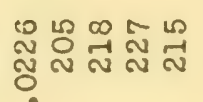 & న్న & 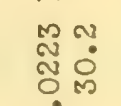 & 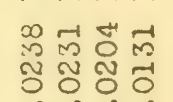 & & F゙ & 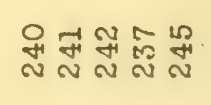 & 倠尔 \\
\hline 娄 & 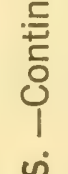 & 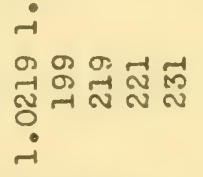 & 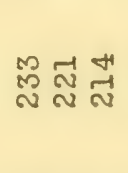 & 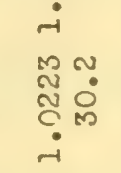 & 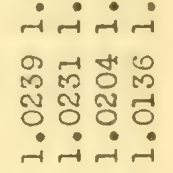 & 帒 & $\begin{array}{l}\dot{-} \\
\stackrel{+}{+} \\
\stackrel{\infty}{0} \\
\dot{-}\end{array}$ & 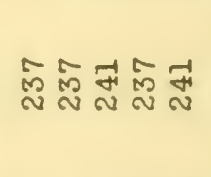 & $\begin{array}{l}\text { m心 } \\
\text { 心 } \\
心 \\
心\end{array}$ \\
\hline$\frac{3}{3}$ & $\sum_{\Sigma}^{n}$ & 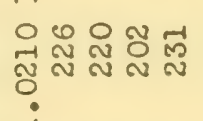 & 藏 & $\begin{array}{l}\widetilde{N} \\
\widetilde{N} \\
0 \\
0\end{array}$ & 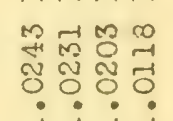 & $\begin{array}{l}\text { 오 } \\
\text { n }\end{array}$ & $\begin{array}{l}\text { Oั } \\
\text { Oे }\end{array}$ & 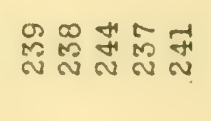 & Ұૈ요요 \\
\hline 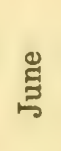 & ż & 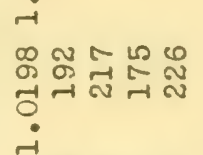 & 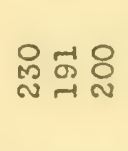 & 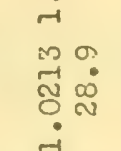 & 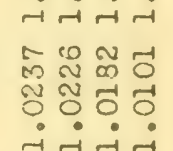 & 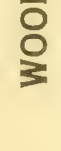 & $\begin{array}{l}-1 \\
\text { Oे } \\
\text { ปั } \\
0\end{array}$ & 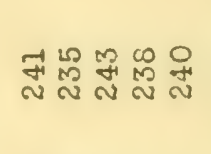 & 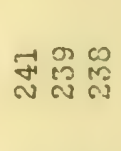 \\
\hline 密 & & 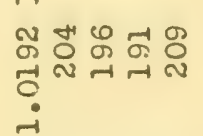 & 悹 & 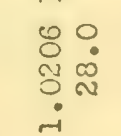 & 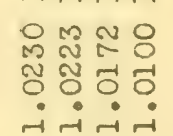 & & 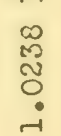 & 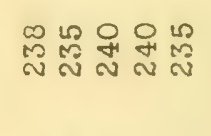 & 앴 \\
\hline 安 & & 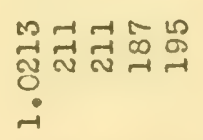 & 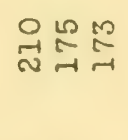 & 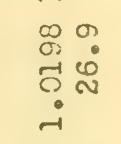 & 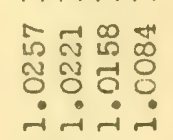 & & 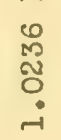 & 心ి & 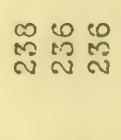 \\
\hline 厽 & & 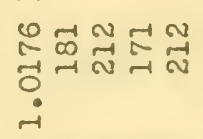 & 是点点 & $\begin{array}{l}\sigma \\
\sigma \\
\vec{a} \\
\vec{j} \\
\dot{i}\end{array}$ & 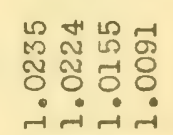 & & 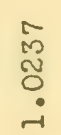 & 怸忍㤀 & 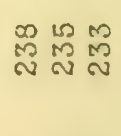 \\
\hline$\dot{0}$ & & 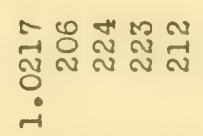 & 式怘芯 & 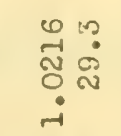 & 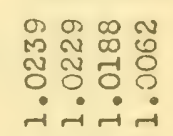 & & $i$ & 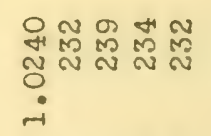 & 焉 \\
\hline กี่ & & 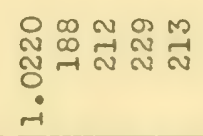 & 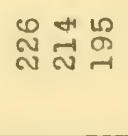 & 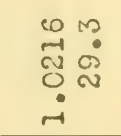 & 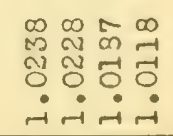 & & $i$ & 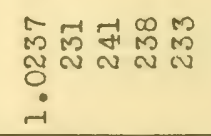 & 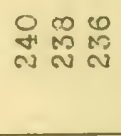 \\
\hline 芠 & & 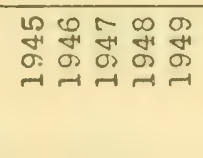 & 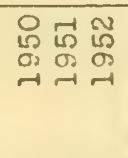 & 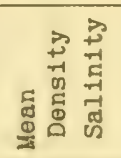 & 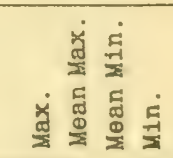 & & $\underset{\sim}{\stackrel{\varpi}{\sigma}}$ & 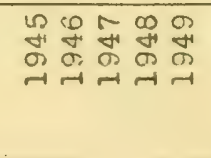 & 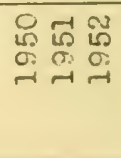 \\
\hline
\end{tabular}




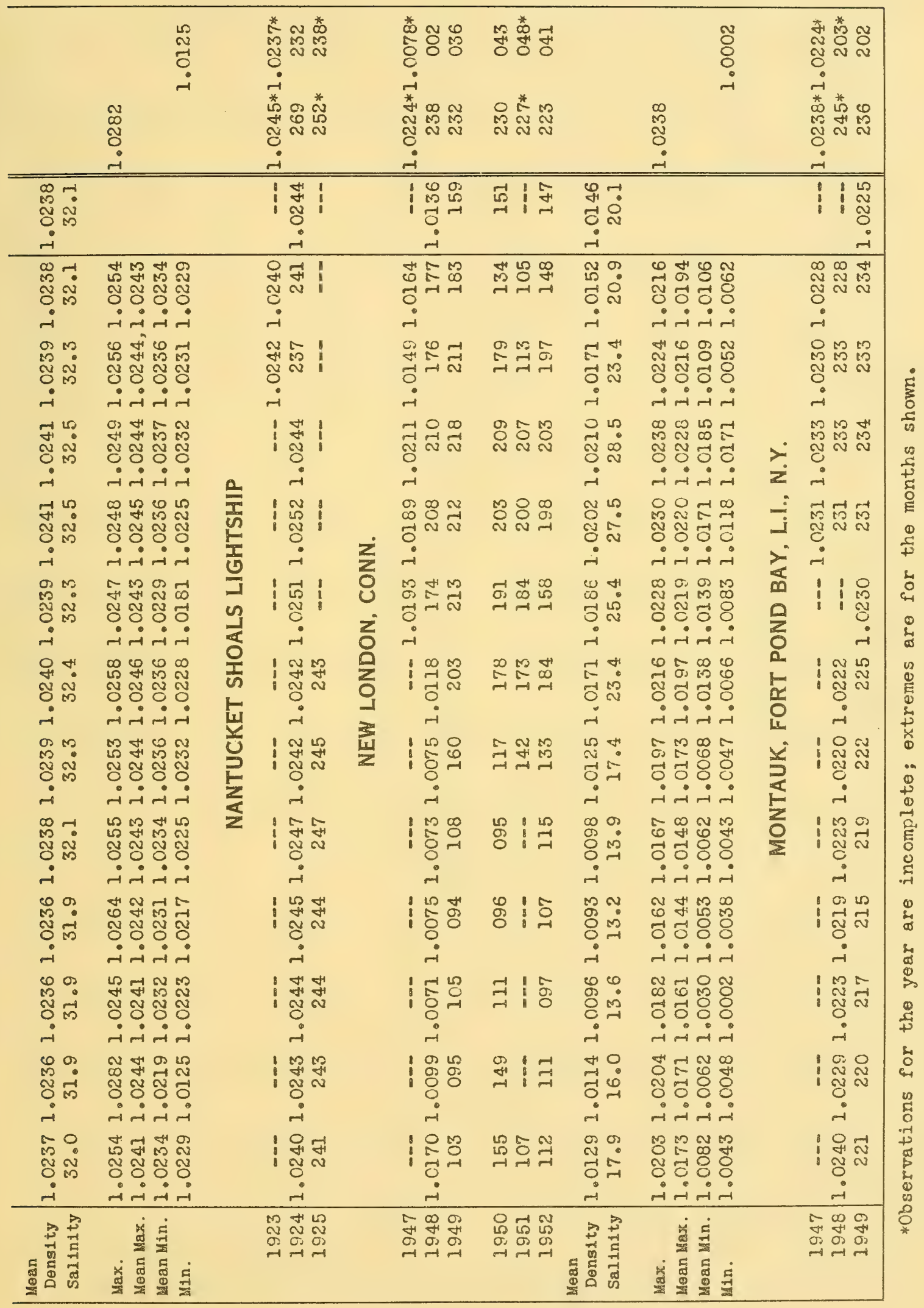




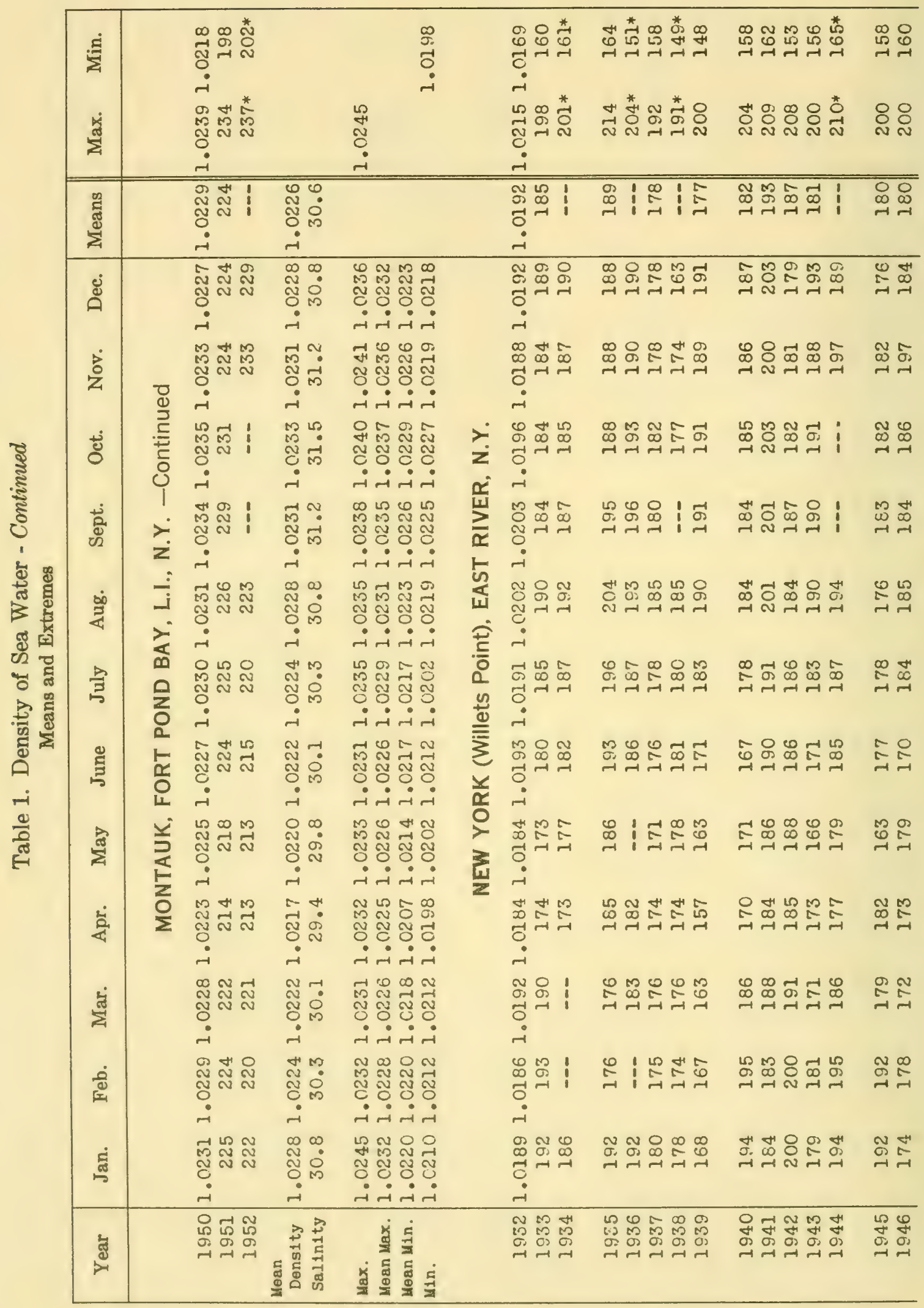




\begin{tabular}{|c|c|c|c|c|c|c|c|c|}
\hline 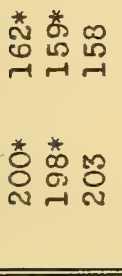 & 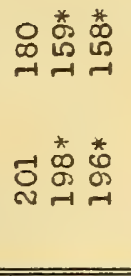 & & $\begin{array}{l}\text { no } \\
\text { ปี } \\
0 \\
-1 \\
\end{array}$ & & 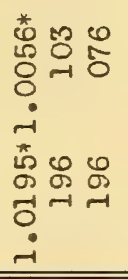 & M & 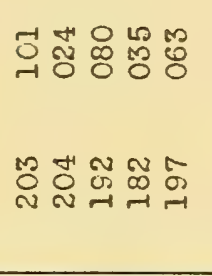 & 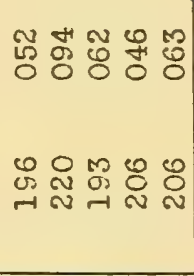 \\
\hline 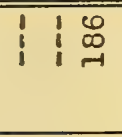 & 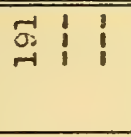 & $\begin{array}{l}+1 \\
\infty \\
-1 \\
0 \\
0 \\
-1\end{array}$ & & & $\begin{array}{l}10 \\
10 \\
1 \\
1 \\
0 \\
1\end{array}$ & 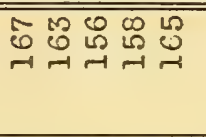 & ঢ0 & 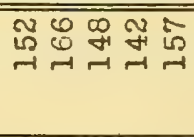 \\
\hline i요요 & $\begin{array}{lll}\leftarrow & -1 & \infty \\
-1 & \infty & \infty\end{array}$ & $\begin{array}{ll}5 & 10 \\
0 & 10 \\
0 & 0 \\
-1 & 0\end{array}$ & 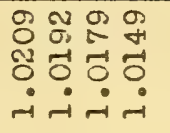 & & 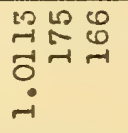 & 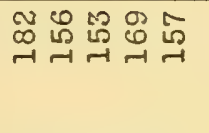 & 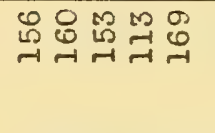 & 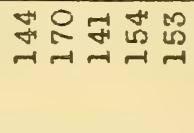 \\
\hline 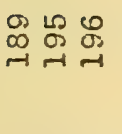 & 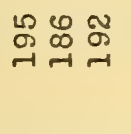 & $\begin{array}{l}\infty \\
\infty \\
\infty \\
\overrightarrow{0} \\
0 \\
\dot{-1}\end{array}$ & 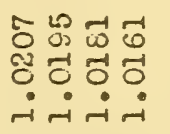 & & $\begin{array}{l}\infty \\
-1 \\
-1 \\
0\end{array}$ & 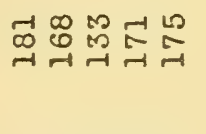 & 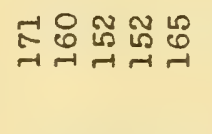 & 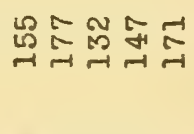 \\
\hline 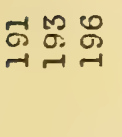 & ボ & $\begin{array}{l}0 \\
\infty \\
\infty \\
-1 \\
\dot{-1}\end{array}$ & 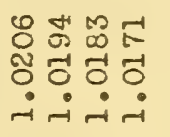 & & $\begin{array}{l}m a \infty \\
0 \\
0 \\
0 \\
0 \\
-i\end{array}$ & 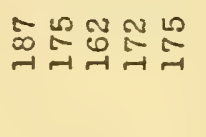 & 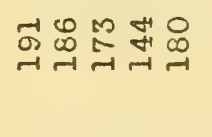 & 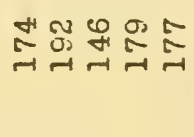 \\
\hline 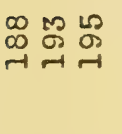 & $\begin{array}{l:l}4 & 1 \\
\sigma & \infty \\
-1 & \infty\end{array}$ & $\begin{array}{l}\circ \\
\text { o } \\
-1 \\
0 \\
0\end{array}$ & 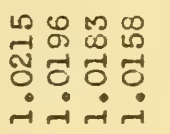 & $\stackrel{i}{i}$ & $\begin{array}{l}\infty \\
0 \\
0 \\
0 \\
0 \\
0 \\
0\end{array}$ & 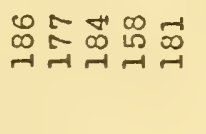 & 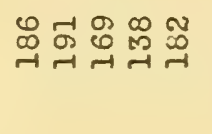 & 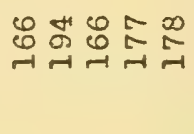 \\
\hline $\begin{array}{c}\infty \\
\infty \\
\infty \\
-1 \\
\infty \\
-1\end{array}$ & $\underset{\sigma}{\sigma}: \mid$ & $\begin{array}{l}0 \\
8 \\
-1 \\
0 \\
0 \\
-1\end{array}$ & 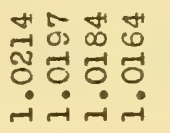 & 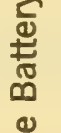 & \begin{tabular}{l}
$-1+\infty$ \\
\multirow{2}{*}{$+\infty$} \\
-1 \\
0 \\
-1
\end{tabular} & 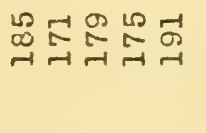 & 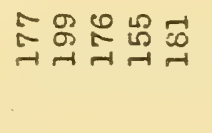 & 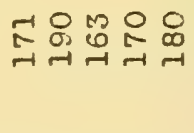 \\
\hline 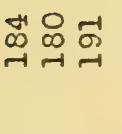 & 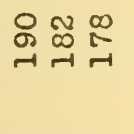 & $\begin{array}{ll}w & v \\
\infty & : \\
-1 & w \\
0 & 0 \\
-1\end{array}$ & 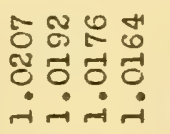 & $\begin{array}{l}E \\
E \\
\underline{y}\end{array}$ & 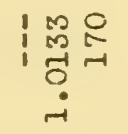 & 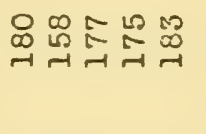 & 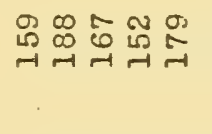 & 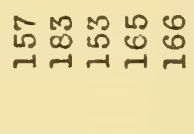 \\
\hline 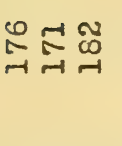 & $\begin{array}{l}\sigma= \\
\infty \\
\infty\end{array}$ & \begin{tabular}{ll}
0 & 0 \\
0 & 0 \\
0 & \multirow{1}{*}{} \\
0 &
\end{tabular} & 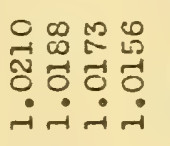 & $\begin{array}{l}0 \\
z \\
\frac{3}{z}\end{array}$ & 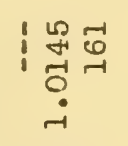 & 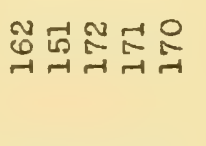 & 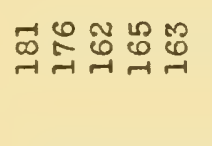 & 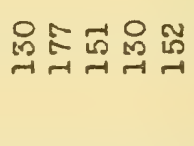 \\
\hline 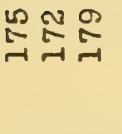 & 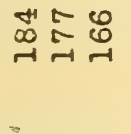 & $\begin{array}{l}0 \\
\dot{0} \\
0 \\
0\end{array}$ & 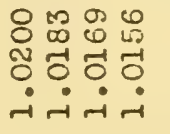 & & 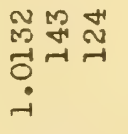 & 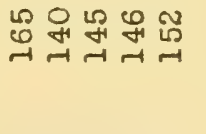 & 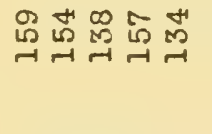 & 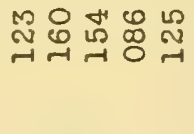 \\
\hline 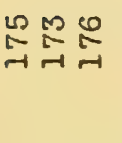 & 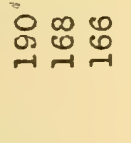 & $\begin{array}{l}0 \\
\stackrel{-1}{0} \\
0\end{array}$ & 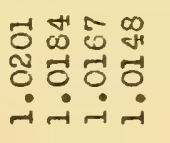 & & 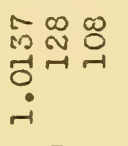 & 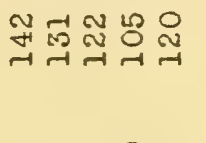 & 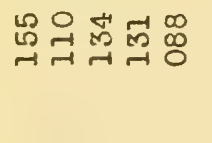 & 芯忍芯命 \\
\hline 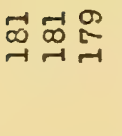 & 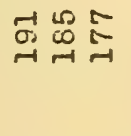 & $\begin{array}{l}-15 \\
\infty \\
-1 \\
0 \\
-1\end{array}$ & 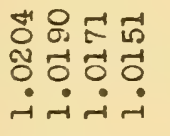 & & $\begin{array}{l}000 \\
0 \\
0 \\
0 \\
0 \\
-1\end{array}$ & 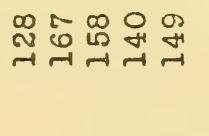 & 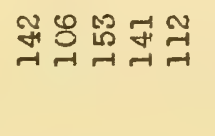 & 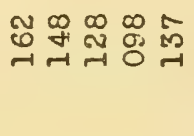 \\
\hline מิ & 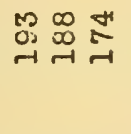 & $\begin{array}{l}\text { मे } \\
1 \\
-1 \\
0 \\
0\end{array}$ & 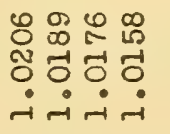 & & 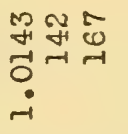 & 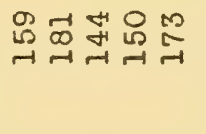 & 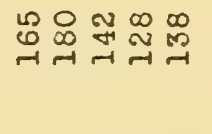 & 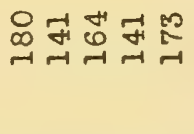 \\
\hline 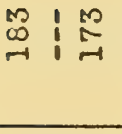 & 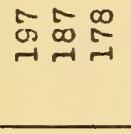 & $\begin{array}{l}n \\
\infty \\
0 \\
0 \\
0 \\
0 \\
0\end{array}$ & 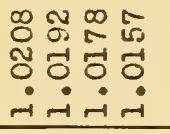 & & 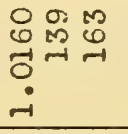 & 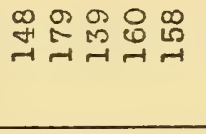 & 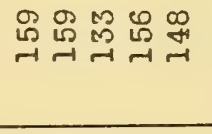 & 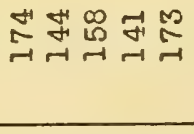 \\
\hline 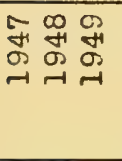 & 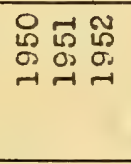 & 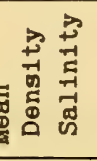 & 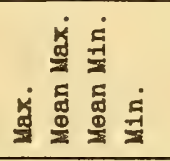 & & 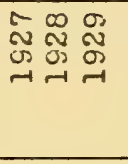 & 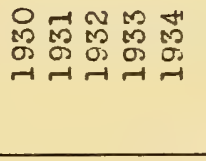 & 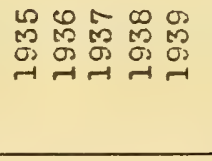 & 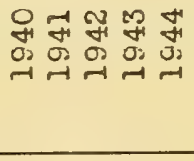 \\
\hline
\end{tabular}




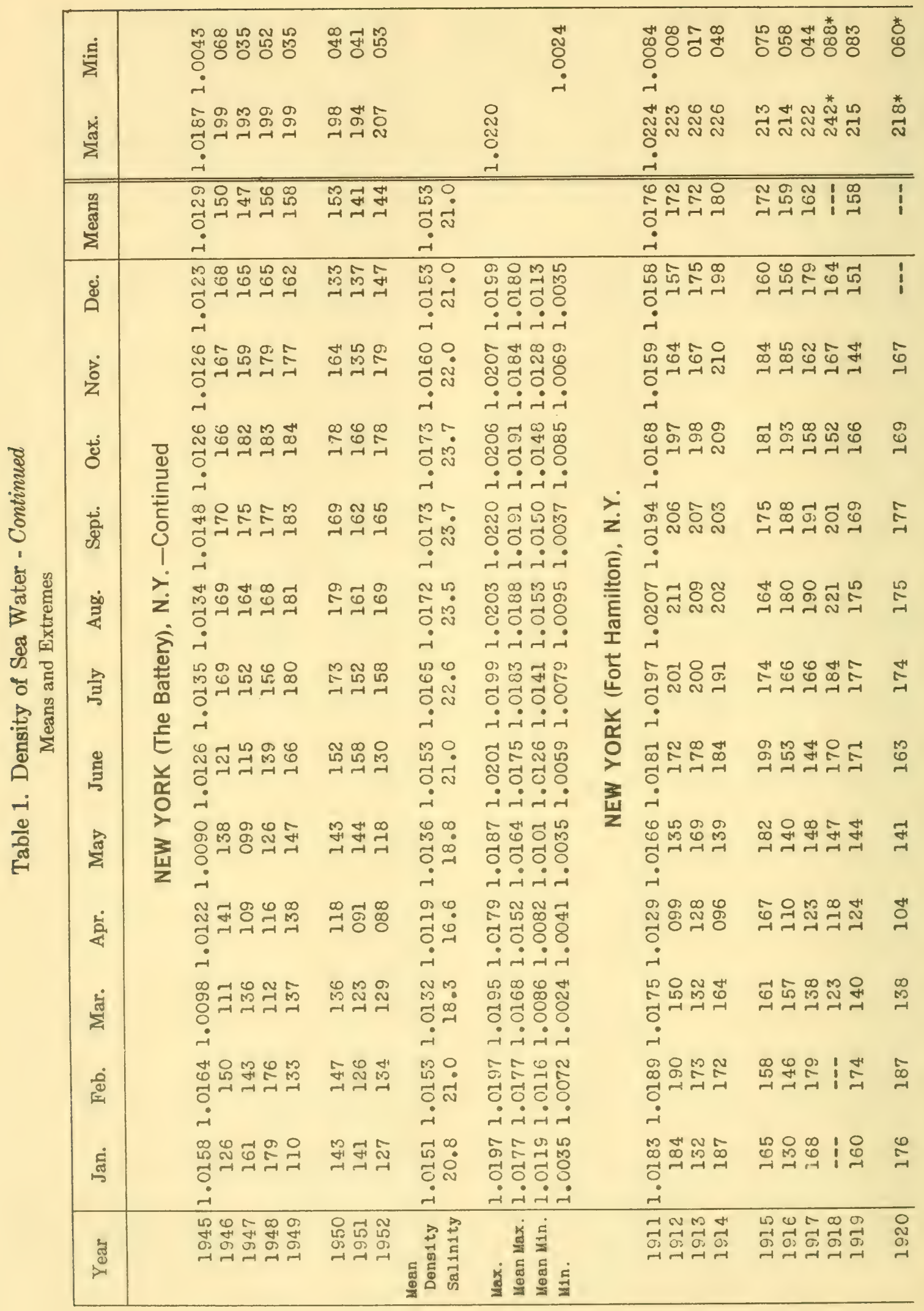




\begin{tabular}{|c|c|c|c|c|c|c|c|c|}
\hline 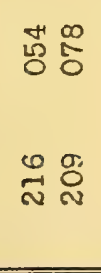 & & 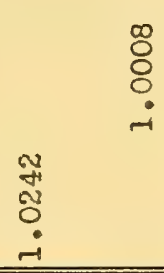 & & 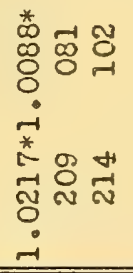 & 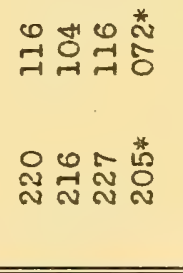 & 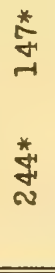 & 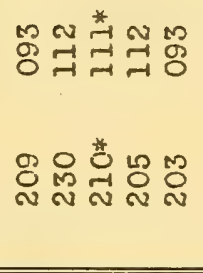 & 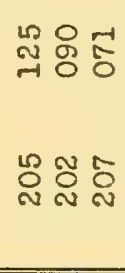 \\
\hline $\begin{array}{ll}\mathbb{N} & 0 \\
0 & 0 \\
-1 & 0-1\end{array}$ & $\begin{array}{l}50 \\
0 \\
0 \\
0 \\
-1\end{array}$ & & & $\begin{array}{lll}1 & 10 & 10 \\
1 & 0 & 0 \\
1 & -1 \\
0 & -1 \\
-1\end{array}$ & 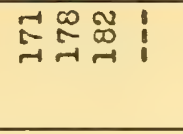 & i & 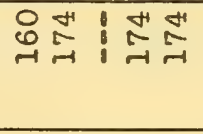 & 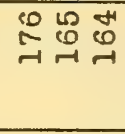 \\
\hline$\underset{\sim}{\infty} \underset{\sim}{\infty}$ & 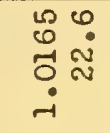 & 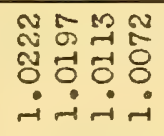 & & 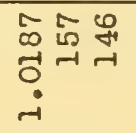 & 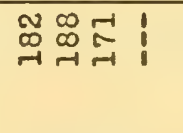 & $\stackrel{0}{2}$ & 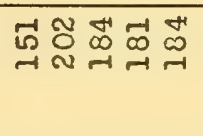 & 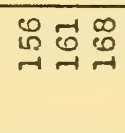 \\
\hline 요 & $\begin{array}{ll}\infty & 0 \\
0 & 0 \\
-1 & 0 \\
0 & 0 \\
-1 & \end{array}$ & 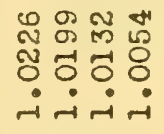 & & 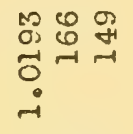 & 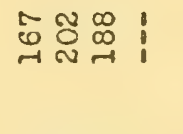 & 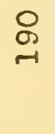 & 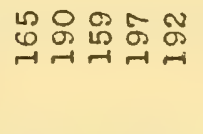 & 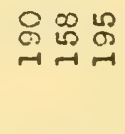 \\
\hline 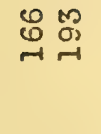 & $\begin{array}{l}0 \\
0 \\
0 \\
0 \\
0\end{array}$ & 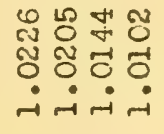 & & 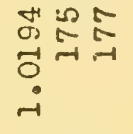 & 옥역 & $\begin{array}{l}\sigma_{1} \\
\sigma \\
-1\end{array}$ & 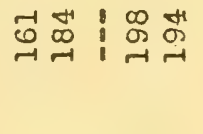 & $\begin{array}{lll}M & 1 & \sigma \\
0 & \infty & \infty \\
-1 & -1 & -1\end{array}$ \\
\hline 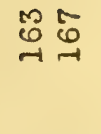 & 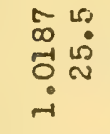 & 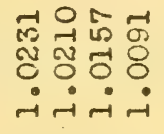 & ; & $\begin{array}{lll}m & 0 & m \\
\infty & \infty & \infty \\
-1 & -1 & -1 \\
0 & & \\
-1 & \end{array}$ & 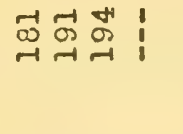 & $\stackrel{\sigma}{\sigma}$ & 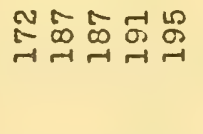 & 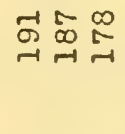 \\
\hline 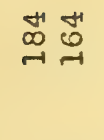 & $\begin{array}{ll}0 & 0 \\
0 & 0 \\
0 & 0 \\
0 & 0 \\
-1\end{array}$ & 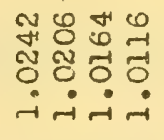 & $\begin{array}{l}z \\
y \\
0\end{array}$ & 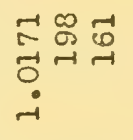 & 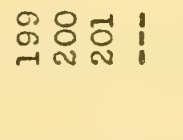 & రి & た & बून \\
\hline${ }_{\substack{0 \\
-1}}^{-15}$ & $\begin{array}{l}-1 \\
\infty \\
0 \\
0-1\end{array}$ & 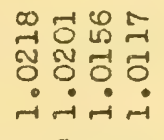 & 폴 & $\begin{array}{l}-1 \\
0 \\
0 \\
0 \\
0 \\
0 \\
-1\end{array}$ & 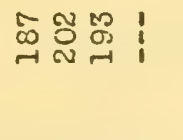 & $\underset{\sim}{\tilde{\sigma}}$ & 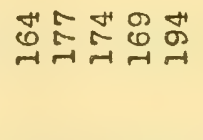 & $\stackrel{\infty}{\infty} \underset{\infty}{\infty}$ \\
\hline$\underset{⿱}{\stackrel{4}{a}}$ & $\begin{array}{l}0 \text { m } \\
0 \\
0 \\
0\end{array}$ & 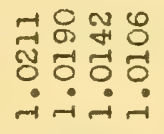 & ज & $\begin{array}{l}\sigma \\
0 \\
0 \\
0 \\
0\end{array}$ & 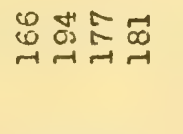 & ! & 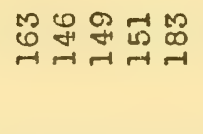 & $\underset{\sim}{\infty} \stackrel{0}{\sim}$ \\
\hline $\begin{array}{c}-1 \\
0 \\
-1\end{array}$ & 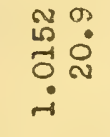 & 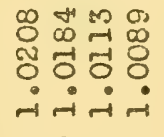 & & 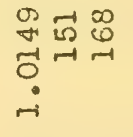 & 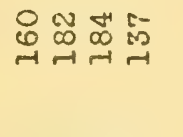 & i & 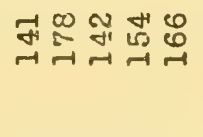 & 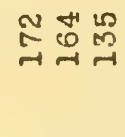 \\
\hline 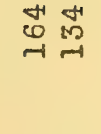 & 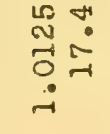 & 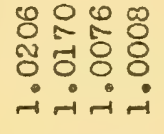 & & 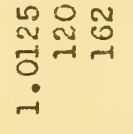 & 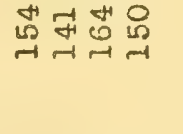 & $i$ & 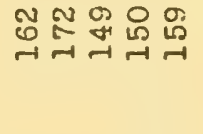 & 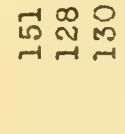 \\
\hline 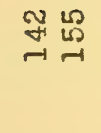 & 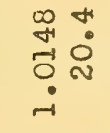 & 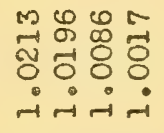 & & $\frac{\pi}{0}$ & 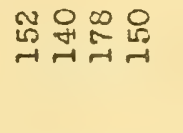 & i & 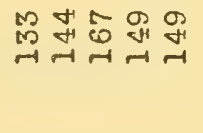 & 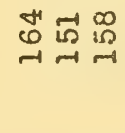 \\
\hline $\begin{array}{l}00 \\
5 \\
\Rightarrow\end{array}$ & $\begin{array}{l}n+1 \\
0 \\
0 \\
0\end{array}$ & 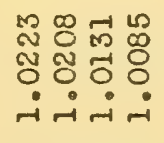 & & $\begin{array}{l}100 \\
10 \\
0 \\
0 \\
-1\end{array}$ & 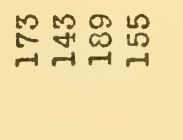 & i & 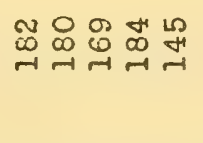 & कृ \\
\hline$\underset{\varpi}{\varpi} \underset{\leftarrow}{\infty}$ & 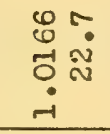 & 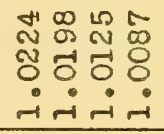 & & $\underbrace{M}_{0}$ & 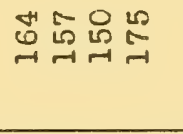 & i & 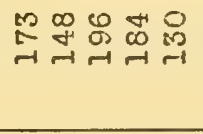 & 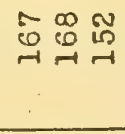 \\
\hline $\begin{array}{l}\tilde{N} \\
\stackrel{\infty}{\sim} \\
\sigma \\
\sigma \\
\sim \\
\sim\end{array}$ & 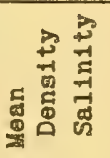 & 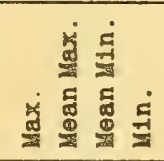 & & $\begin{array}{lll}\kappa & \infty & 0 \\
0 & 0 & \infty \\
\omega & \infty & \infty \\
-1 & -1\end{array}$ & 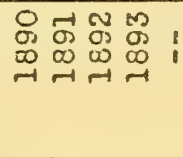 & $\underset{+1}{+4}$ & 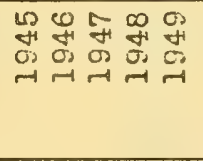 & 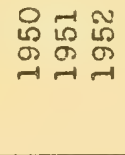 \\
\hline
\end{tabular}




\begin{tabular}{|c|c|c|c|c|c|c|c|c|c|c|}
\hline 离 & & & $\begin{array}{l}-1 \\
0 \\
0 \\
-1\end{array}$ & & 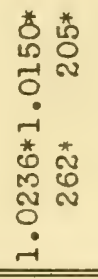 & 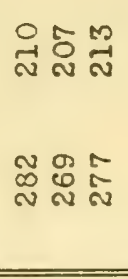 & & 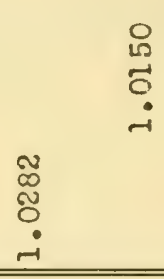 & & 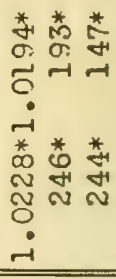 \\
\hline 范 & & $\begin{array}{l}-1 \\
0 \\
0 \\
0\end{array}$ & & & $1 !$ & 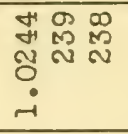 & 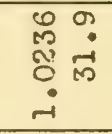 & & & \begin{tabular}{l|l} 
& 1
\end{tabular} \\
\hline ஜूँ & & $\begin{array}{l}m 5 \\
-1 \\
0 \\
0\end{array}$ & 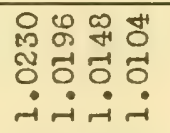 & & $\begin{array}{l}1 \\
1 \\
\text { I } \\
0 \\
-1\end{array}$ & 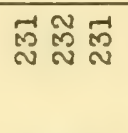 & 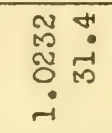 & 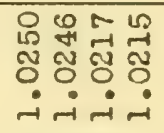 & & 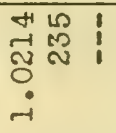 \\
\hline 客 & & $\begin{array}{ll}0 & 0 \\
\infty & 0 \\
-1 & \dot{1} \\
0 & \sim \\
-1 & \end{array}$ & 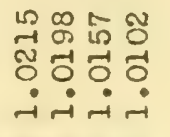 & & $\begin{array}{l}m \\
1 \\
0 \\
0 \\
0 \\
1\end{array}$ & 矛恕 & $\begin{array}{ll}0 & \sigma \\
0 & \dot{\infty} \\
0 & \end{array}$ & 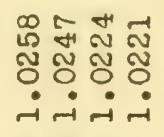 & & 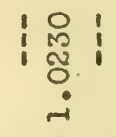 \\
\hline ह் & & $\begin{array}{l}-5 \\
\infty \\
-1 \\
0 \\
0 \\
-1\end{array}$ & 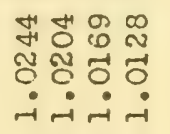 & & $\begin{array}{l}\text { + } 0 \\
\text { N } \\
\text { 心 } \\
0\end{array}$ & 范志 & $\begin{array}{l}\text { w } \\
\text { N } \\
\text { Oे } \\
0 \\
\dot{-1}\end{array}$ & 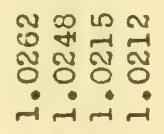 & & 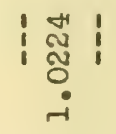 \\
\hline के & 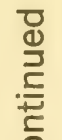 & $\begin{array}{l}0 \\
0 \\
0 \\
0 \\
0\end{array}$ & 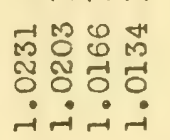 & ri & 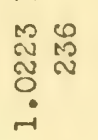 & น & 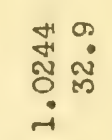 & 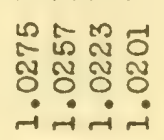 & ְִ & 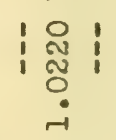 \\
\hline$\stackrel{\infty}{4}$ & $\begin{array}{c}\overline{0} \\
1 \\
\text { ¿ }\end{array}$ & 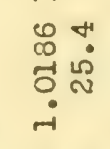 & 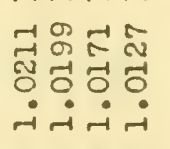 & $\begin{array}{l}z \\
\frac{1}{U} \\
z\end{array}$ & $\begin{array}{l}\tilde{a} \\
\vec{\sim} \\
\tilde{\sigma}\end{array}$ & $\left.\begin{array}{l}-1 \\
6 \\
\sim\end{array}\right)$ & 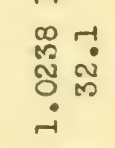 & 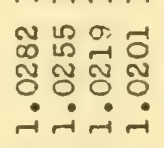 & $\frac{z}{z}$ & 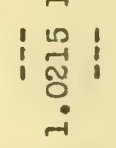 \\
\hline$\stackrel{3}{3}$ & $\begin{array}{l}z \\
y \\
0 \\
0\end{array}$ & $\begin{array}{ll}4 & -1 \\
0 & 0 \\
0 & 0 \\
0 & 0\end{array}$ & 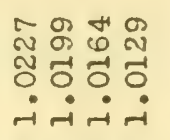 & $\begin{array}{l}\underset{\frac{\pi}{\alpha}}{\infty} \\
\text { v } \\
\sum\end{array}$ & \begin{tabular}{l}
$m$ \\
$\stackrel{+}{+}$ \\
\multirow{U}{*}{} \\
0 \\
-1
\end{tabular} & 용 志 & 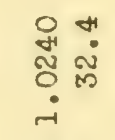 & 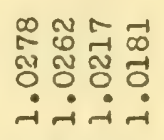 & $\frac{2}{2}$ & 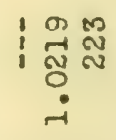 \\
\hline$\stackrel{\Xi}{\Xi}$ & $\frac{1}{2}$ & $\begin{array}{l}\pi \\
0 \\
0 \\
0 \\
\dot{-1}\end{array}$ & 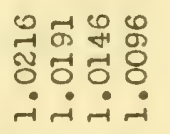 & 이 & $\begin{array}{l}\infty \\
0 \\
0 \\
0 \\
0 \\
-1\end{array}$ & 芯苾塄 & $\begin{array}{l}\infty \\
\tilde{N} \\
\tilde{0} \\
\dot{0} \\
\dot{-1}\end{array}$ & 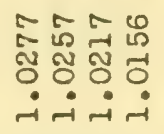 & $\bar{n}$ & 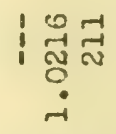 \\
\hline 窄 & ஸ & 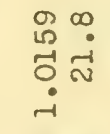 & 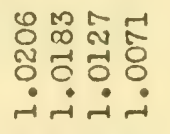 & & $\begin{array}{l}0 \\
0 \\
0 \\
0 \\
0 \\
0\end{array}$ & 筧尔 & $\begin{array}{l}\text { N } \\
\text { Uै } \\
\dot{0}\end{array}$ & 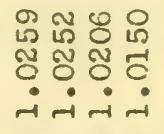 & & 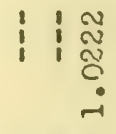 \\
\hline 客 & & 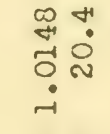 & 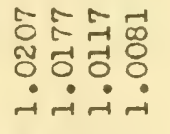 & & 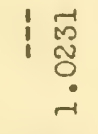 & 芯 융 & $\begin{array}{l}\text { w } 0 \\
\text { तै } \\
0 \\
0\end{array}$ & 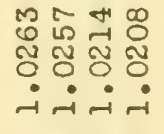 & & 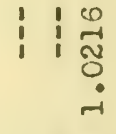 \\
\hline$\sum_{\Sigma}^{\stackrel{5}{\Sigma}}$ & & $\begin{array}{l}\text { H' } \\
\dot{0} \\
\dot{0}\end{array}$ & 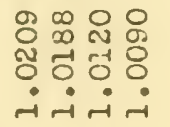 & & $\begin{array}{l}n \\
1 \\
0 \\
0 \\
0 \\
0\end{array}$ & $\begin{array}{l}\text { D } \\
\text { N } \\
\text { N } \\
\text { N }\end{array}$ & 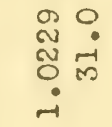 & 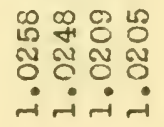 & & $1 \mid \begin{array}{l}0 \\
1 \\
0 \\
0 \\
0 \\
0 \\
0\end{array}$ \\
\hline 这 & & $\begin{array}{l}\qquad 0 \\
0 \\
-1 \\
0 \\
-i\end{array}$ & 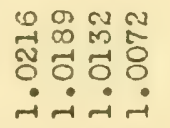 & & $1 !$ & 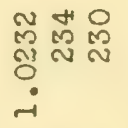 & $\begin{array}{l}\text { N } \\
\text { ñ } \\
\text { U } \\
\text { में }\end{array}$ & 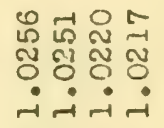 & & | $\begin{array}{l}\mid c \\
0 \\
0 \\
0 \\
0\end{array}$ \\
\hline 㟧 & & 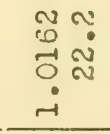 & 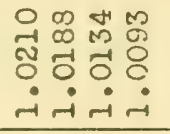 & & 1 & 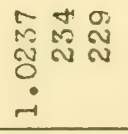 & $\begin{array}{ll}m & n \\
0 & 0 \\
0 & -1 \\
0 & n\end{array}$ & 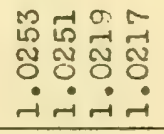 & & 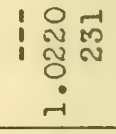 \\
\hline ఫ్తు & & 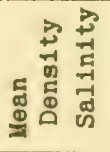 & 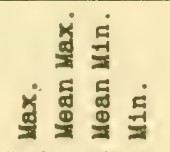 & & 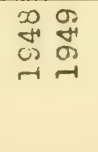 & 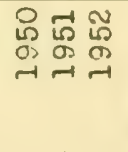 & 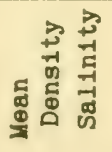 & 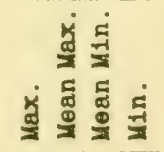 & & 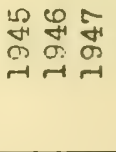 \\
\hline
\end{tabular}




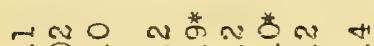

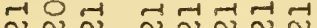
ชิ<smiles>[CH]</smiles>

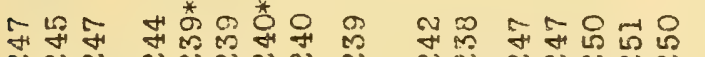
ก $N$

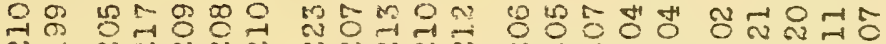

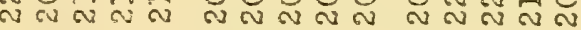

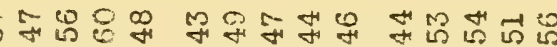

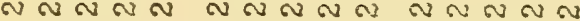

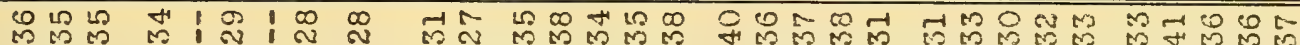
กN -1

ติ

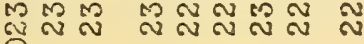

-i

NNE

两

?

ก $15 \leftarrow$ ल

mก

O

$7 \rightarrow$

$\infty \pi$ \% MN

$\cos 200$

$\log \pi$ N

0 -

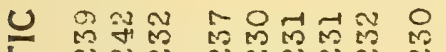

- NUN N

?

मम

西出

i-

H

恣 N

O

i-

๓ ल मँ

MกN

O

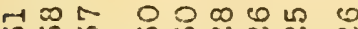
M N M MNNN NUN NNNNN

-

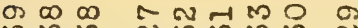

M $\mathrm{N}$ N N N N N N N<smiles>[CH]C[CH]</smiles>

N N N N N N N
๙ N $N$ N $N$ N $N$ N $N$ N $N$ N

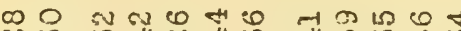

N

$4 \sin 60$

nल N N N N N N N N N N N N N N N N N

M๊ N N N

सing

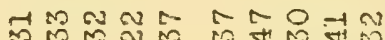

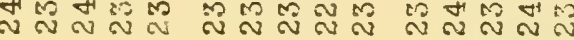

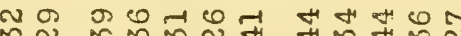

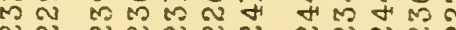
N N N $N$ N ก $N$ ด

m N N N N N N N N N

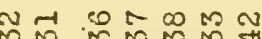
$\mathrm{N} N \stackrel{N}{N} N$

$\because \infty$ is 00

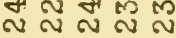

只 N

mo

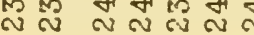

is $\approx \approx \pi$

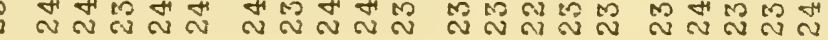

$\ln 4 \infty$ \& $\infty$

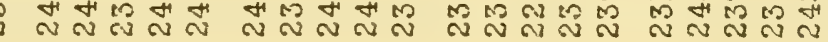

$\rightarrow m \leqslant c m$

$\pi$ m N N N N N N N N

Aก

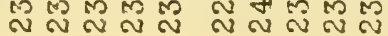

L $N$ L N N N N N

$\sin 2 \pi-1$ N $N$ N

$\operatorname{son} \pi / 4 \pi$

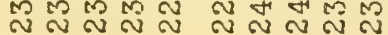

$\infty \omega \pi$. 旅

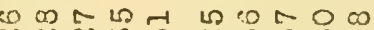

ก๊ N

$\approx \ln \infty$ L $\infty$ N N N N N

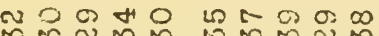
$\mathrm{N} \pi \mathrm{N} N \mathrm{~N} N \mathrm{~N}$

a $\infty N-\infty$ ก

m

Hon

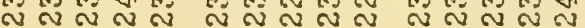

A

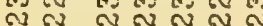

$\pi \infty \pi n$ N N N N N NNNNN $N$ 래

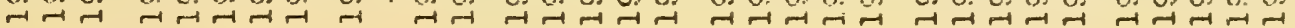




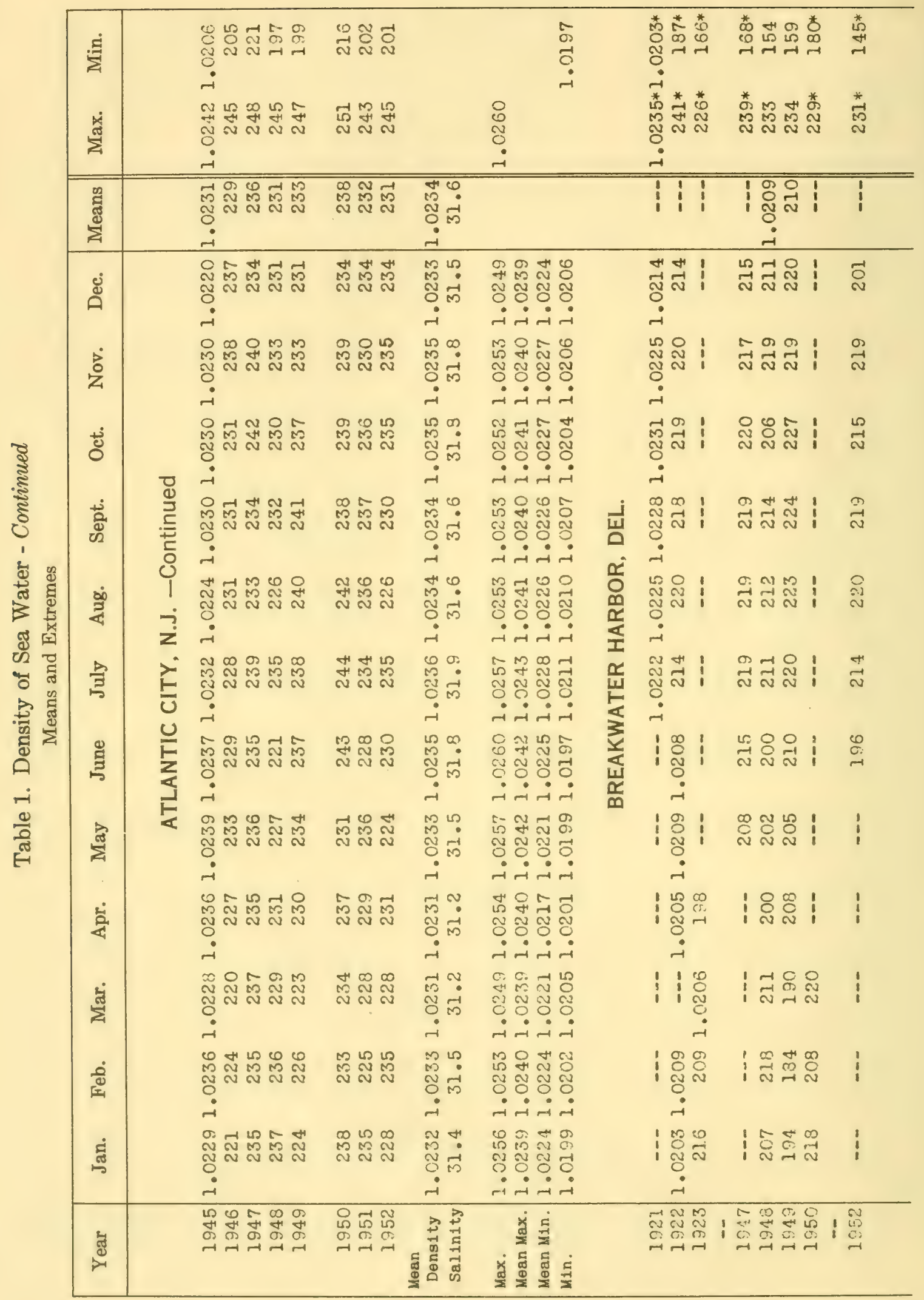




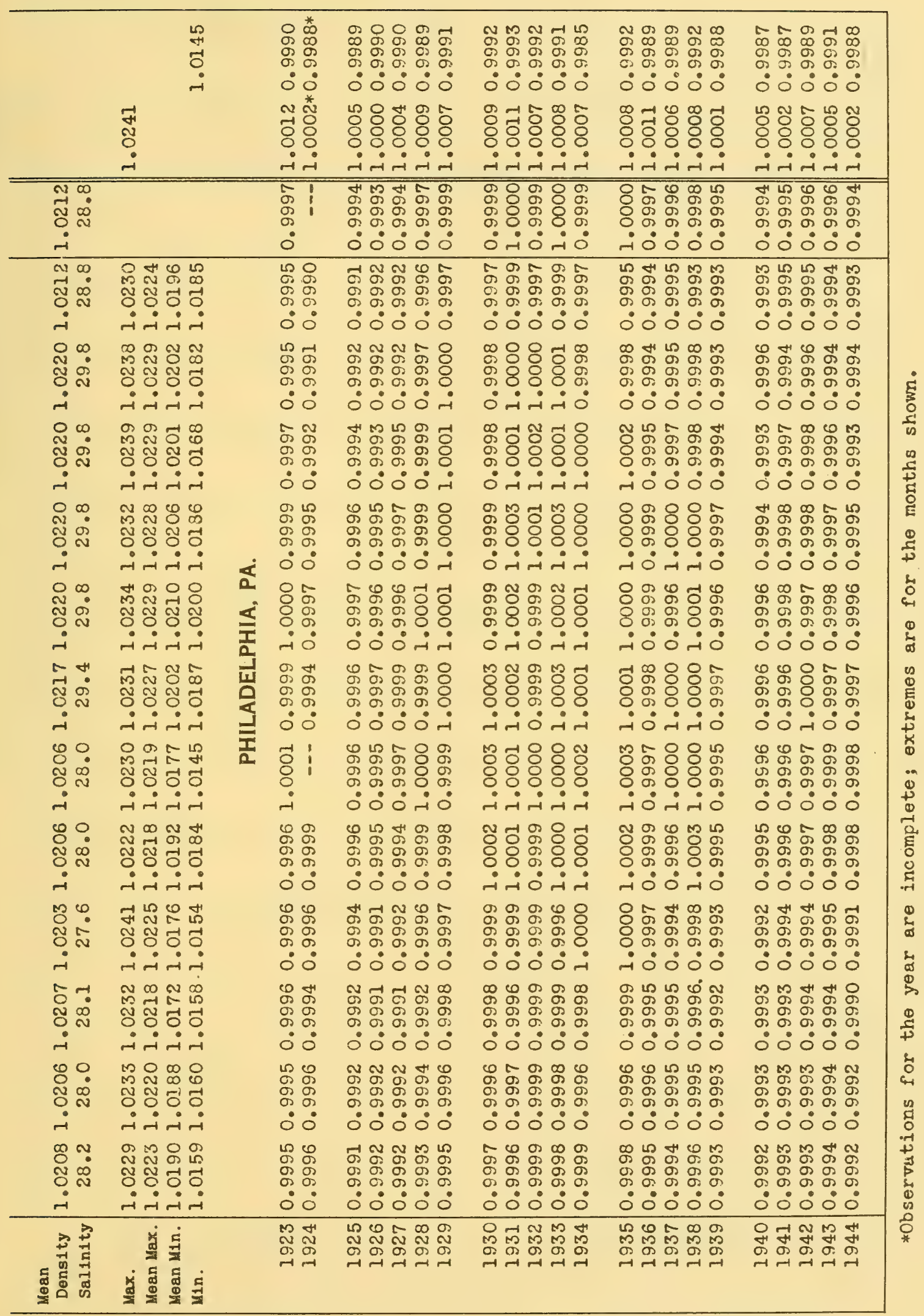




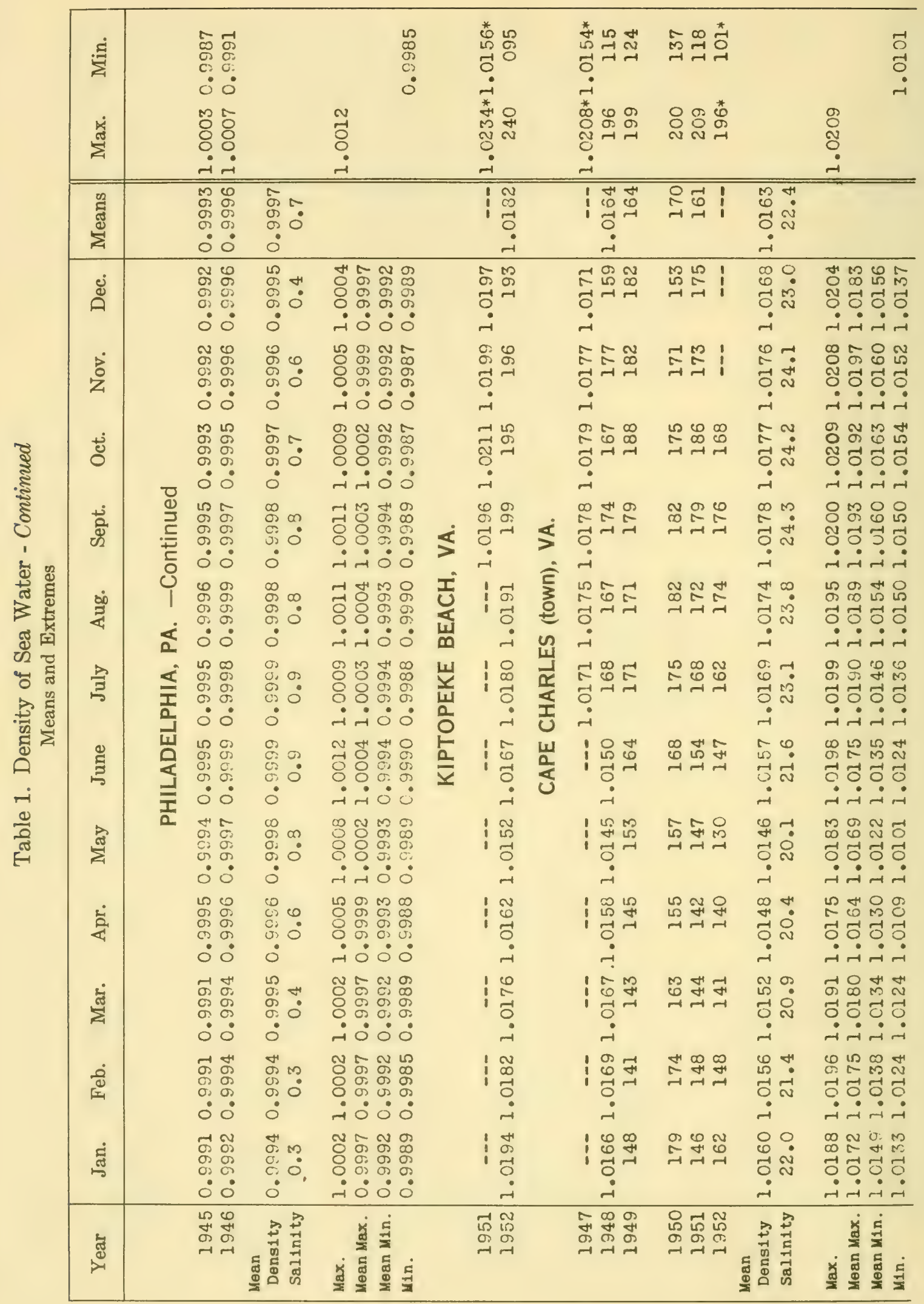




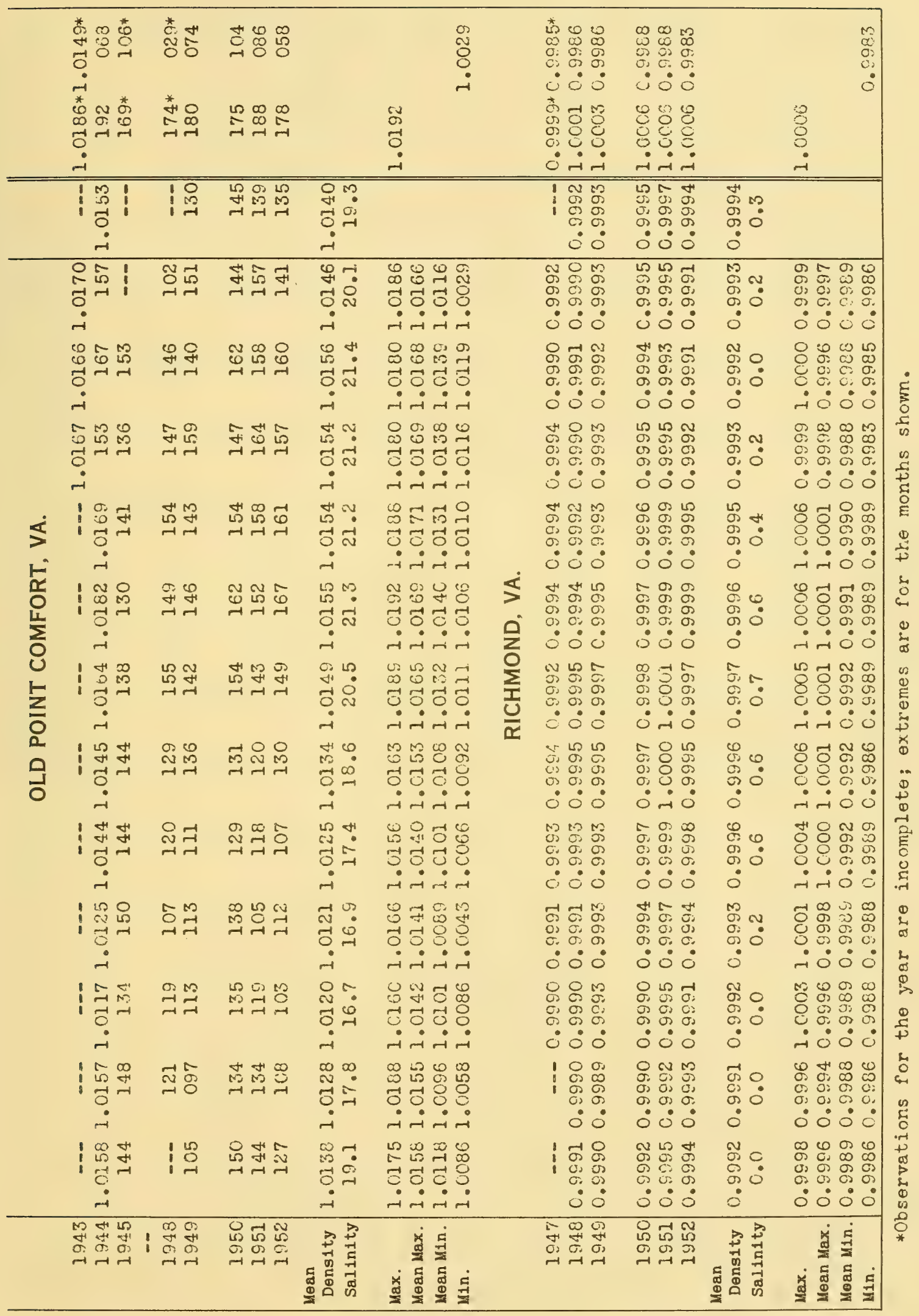




\begin{tabular}{|c|c|c|c|c|c|c|c|c|}
\hline 密 & & 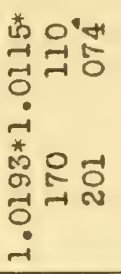 & & 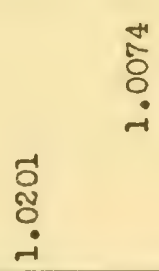 & & 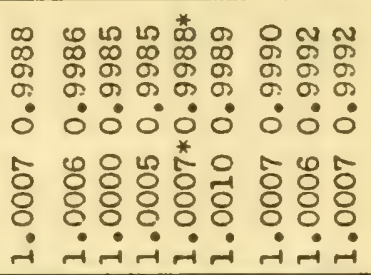 & & $\stackrel{\circ}{\ddot{8}}$ \\
\hline 㝝 & & 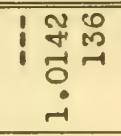 & 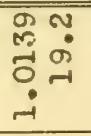 & & & 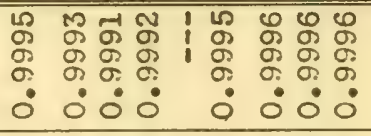 & $\begin{array}{l}2 \\
\stackrel{2}{2} \\
\delta \\
0 \\
0 \\
0\end{array}$ & \\
\hline 迎 & & 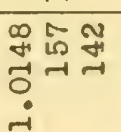 & 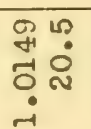 & 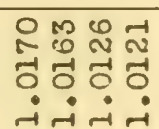 & & 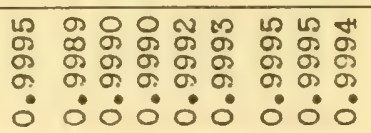 & $\begin{array}{l}\text { ma } \\
\text { Oे } \\
\text { o. } \\
0 \\
0\end{array}$ & 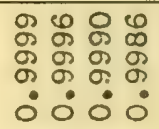 \\
\hline 客 & & 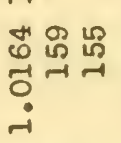 & 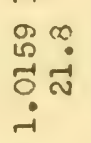 & 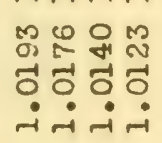 & & 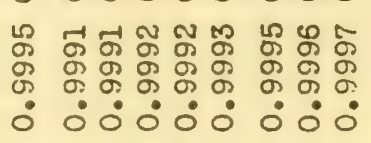 & 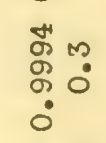 & 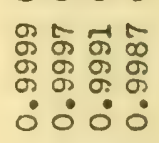 \\
\hline हैं & & 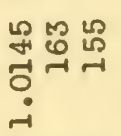 & 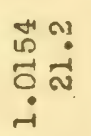 & 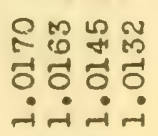 & & 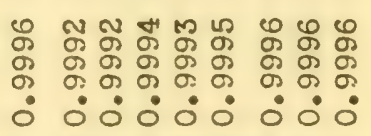 & 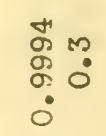 & 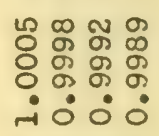 \\
\hline$\dot{0}$ & & 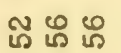 & ? & - & & 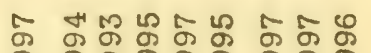 & $\nsubseteq 0$ & $58 \sigma \infty$ \\
\hline षे & 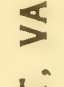 & $\stackrel{-\oplus}{-1}$ & $\overbrace{}^{-1}$ & 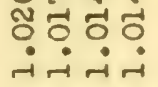 & ن & o & के & 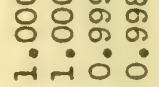 \\
\hline & $\underline{E}$ & น & 品 & 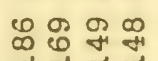 & D & 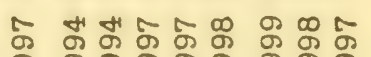 & 5 & 후용ㅇㅇ \\
\hline$\frac{2}{4}$ & 음 & & $\begin{array}{l}\vec{C} \\
\dot{i}^{-1}\end{array}$ & 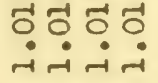 & ż & 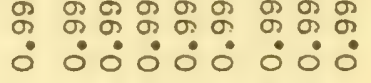 & $\stackrel{9}{\circ} \dot{0}$ & 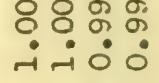 \\
\hline$\triangleq$ & 足 & 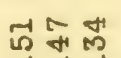 & 乐里 & 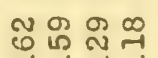 & Uु & సీ & के & 엉 ๘ొ \\
\hline 3 & 氙 & & ${ }_{i}^{-1} \rightarrow$ & ह்َ & $\frac{\check{T}}{\omega}$ & 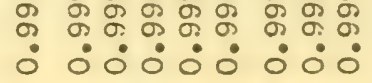 & $\stackrel{9}{\circ}$ & 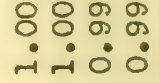 \\
\hline छ & 0 & mat & 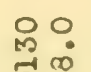 & 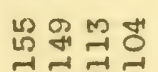 & $\$$ & 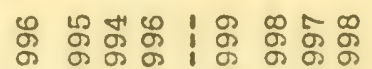 & Sิ? & 융 ర్రి \\
\hline 5 & $\overrightarrow{0}$ & & $i^{-1}$ & iનi & & 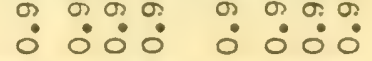 & $\because 0$ & 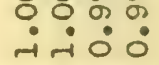 \\
\hline$\Rightarrow$ & & : 용 & Iㅇ. & ติ & & ธらઠ゙の & $\therefore 0$ & జ \\
\hline$\Sigma_{\Sigma}^{\varpi}$ & & & 象 & 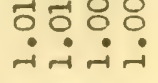 & & 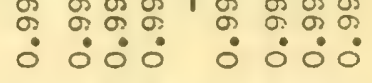 & : & 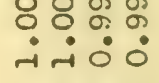 \\
\hline 遅 & & 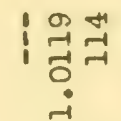 & 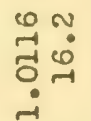 & 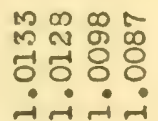 & & 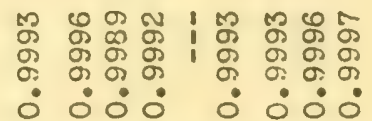 & $\begin{array}{l}\text { में } \\
\text { के: } \\
\vdots \\
0\end{array}$ & 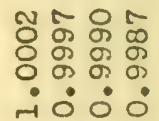 \\
\hline 岕 & & 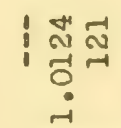 & 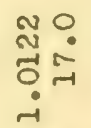 & 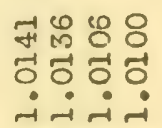 & & 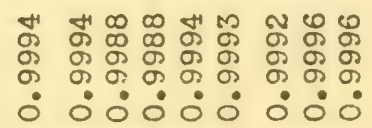 & 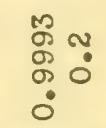 & 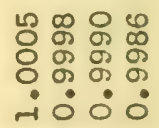 \\
\hline 这 & & 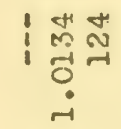 & \begin{tabular}{l}
9 \\
\multirow{3}{*}{$:$} \\
$\vdots$ \\
$\dot{i}$
\end{tabular} & 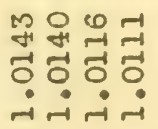 & & 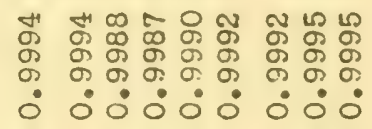 & $\begin{array}{l}\text { के } \\
\text { ळे } \\
\text { ळ. } \\
\dot{0}\end{array}$ & 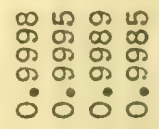 \\
\hline డ్డ & & 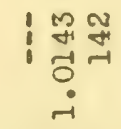 & 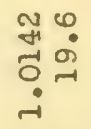 & 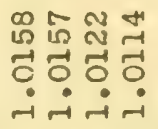 & & 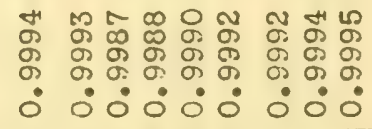 & $\begin{array}{l}\text { Ñ } \\
\text { ळे } \\
\text { : } \\
\dot{0}\end{array}$ & 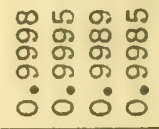 \\
\hline द्र & & 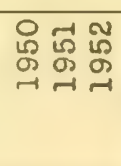 & 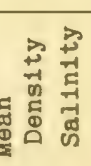 & 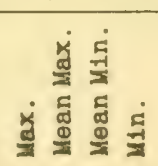 & & 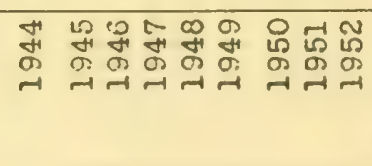 & 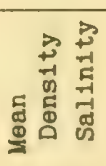 & 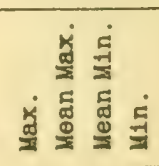 \\
\hline
\end{tabular}




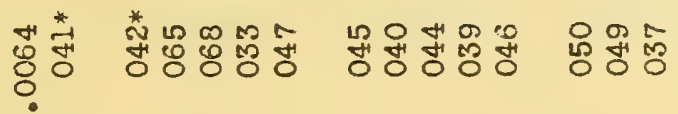

^ี * *

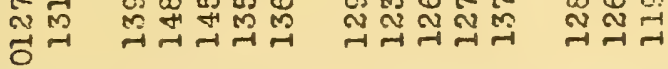

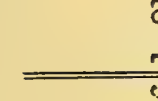

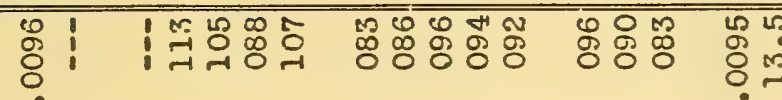

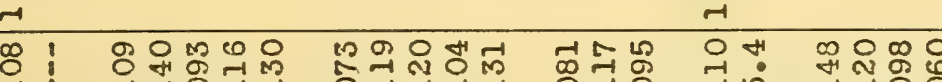

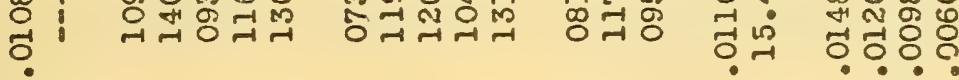

-i

జ 00 ฉ

ก

की

ठन

$\rightarrow$

ஜ대

ठ규 न्न

-

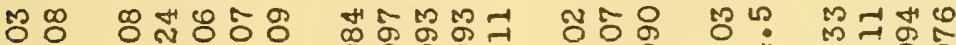

ठㄱ न

(i) $-i$ in-

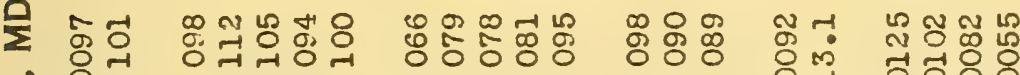

i 8

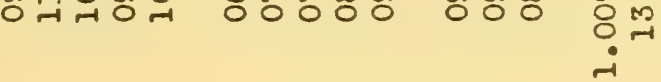

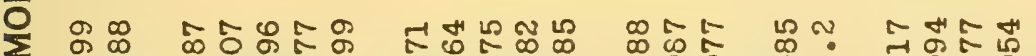

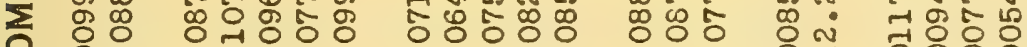

1.

○.

O. $\infty$ -

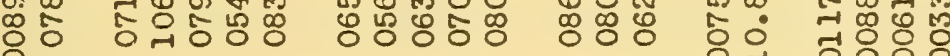
$\because$

.

ک180.

\section{舟}

\%ำ 8

ㄷㅇㅇㅇㅇㅇㅇㅛ -i

.

-i

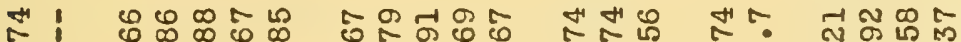

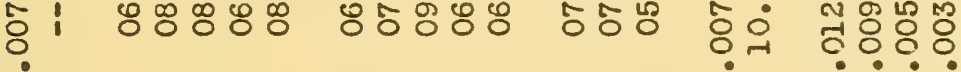

-i

i

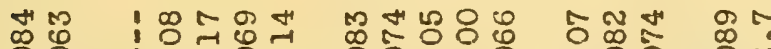

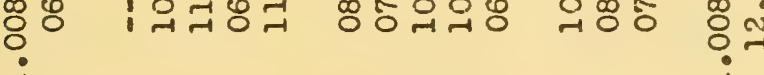

-i

A $\rightarrow-1$

Нี

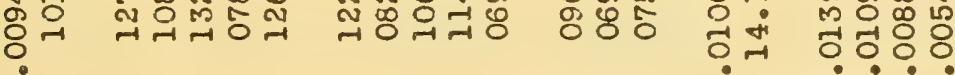

-

$-i$

-i $-1-$

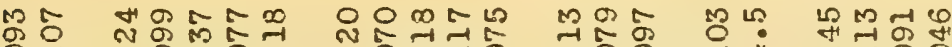

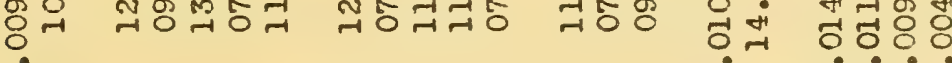

$-1$

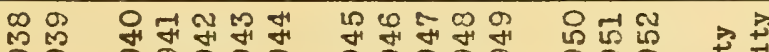

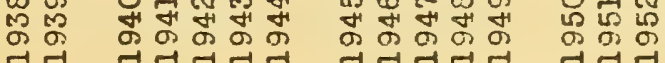

$-$

तनंन्म

药

ภํำ

-

*

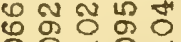

$80-10 \pi$

$-1$

ำำ

8000 $-1$

0 m $m$

ॠั

8

$\pi 4 \pi n$

늉 융 \%

-

क $0 \mathrm{~N}$

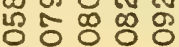

80000

-

H $10 \mathrm{~m} \mathrm{~m}$

ᄂ

$\infty 510$

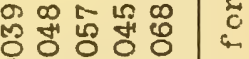

कै

경동ํㅇㅇ

?

-i

m 00

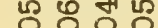

-i

4 00

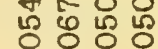

8

$-1$

1 -

กิ

응 550 -

! 蛋紫 ชิ융 -i

요요요 \%

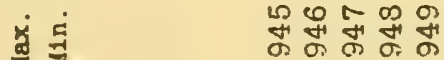

도

ता $\rightarrow-1$

11 


\begin{tabular}{|c|c|c|c|c|c|c|c|c|c|}
\hline 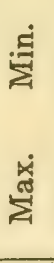 & & 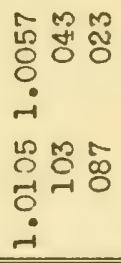 & & $\begin{array}{l}\mathscr{0} \\
0 \\
\dot{0}\end{array}$ & & 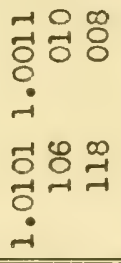 & 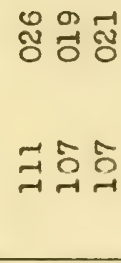 & & $\begin{array}{l}m \\
-1 \\
0 \\
-1 \\
-i\end{array}$ \\
\hline 毠 & & 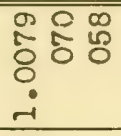 & 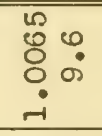 & & & $\begin{array}{l}8 \% \\
80 \% \\
8 \\
-1\end{array}$ & $8 \% 8$ & $\begin{array}{l}\dot{8} \\
\dot{8} \\
0 \dot{0} \\
\dot{-1}\end{array}$ & \\
\hline هั่ & & $\begin{array}{l}0.12 \\
0 \\
0 \\
0\end{array}$ & $\begin{array}{l}0 \\
8 \\
8 \\
0\end{array}$ & $\begin{array}{l}+1 \\
0 \\
0 \\
0\end{array}$ & & 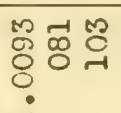 & 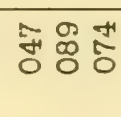 & $\begin{array}{l}-0 \\
0 \\
0\end{array}$ & 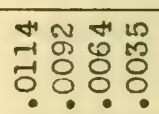 \\
\hline 客 & & 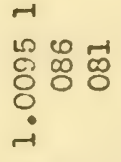 & 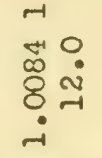 & 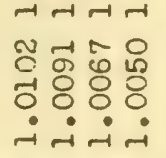 & & $\begin{array}{l}\overrightarrow{1} \\
\tilde{\delta} \\
\tilde{\delta} \\
0 \\
0 \\
\dot{-}\end{array}$ & స్ & $\begin{array}{l}\overrightarrow{1} \\
8 \\
0 \\
0 \\
0 \\
\dot{-1}\end{array}$ & 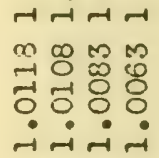 \\
\hline हैं & & 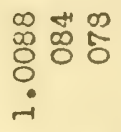 & \begin{tabular}{l}
$\infty$ \\
$\infty$ \\
0 \\
\hdashline \\
$-i$
\end{tabular} & 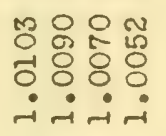 & & 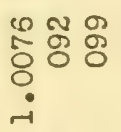 & ஜరం & $\begin{array}{l}8 \\
8 \\
8 \\
\stackrel{\sim}{\sim} \\
\dot{i}\end{array}$ & 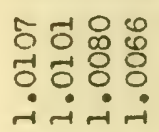 \\
\hline 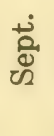 & 兽 & 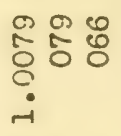 & $\begin{array}{l}00 \\
0: 0 \\
0 \\
0 \\
0-1\end{array}$ & 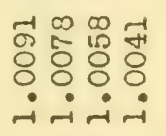 & & 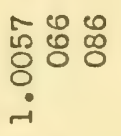 & 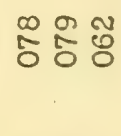 & 落: & 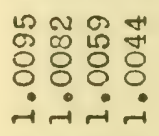 \\
\hline 我 & ن & 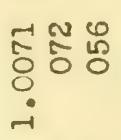 & 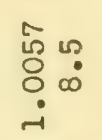 & 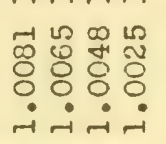 & $\begin{array}{l}\dot{0} \\
\dot{v}\end{array}$ & 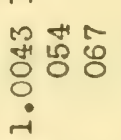 & 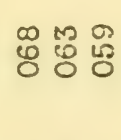 & 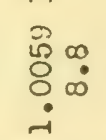 & 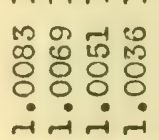 \\
\hline 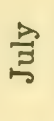 & $\begin{array}{l}\Sigma \\
\text { Uئ }\end{array}$ & $\begin{array}{l}480 \\
8 \% 8 \\
8\end{array}$ & $\begin{array}{l}\infty \\
: 0 \\
0 \\
0 \\
0 \\
\dot{0}\end{array}$ & 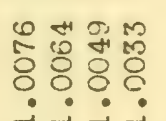 & 할 & 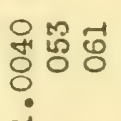 & 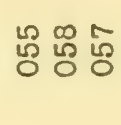 & 我 & 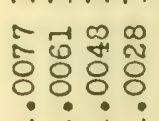 \\
\hline 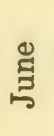 & 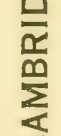 & 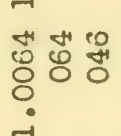 & $\mathscr{\delta}_{0}^{\infty}$ & 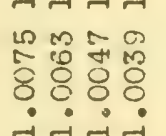 & $<$ & 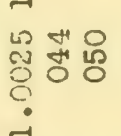 & 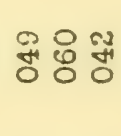 & \&ें & 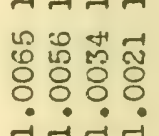 \\
\hline 究 & & 莕 & 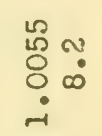 & 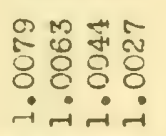 & & 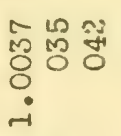 & 뵹융 ్ㅀ & 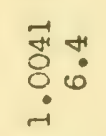 & 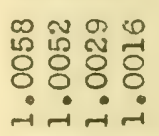 \\
\hline 安 & & 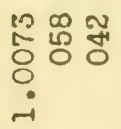 & 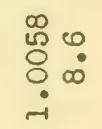 & 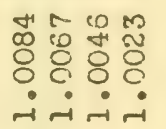 & & 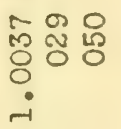 & 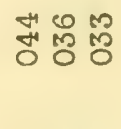 & 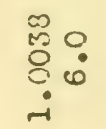 & 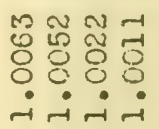 \\
\hline$\sum_{\Sigma}^{\stackrel{\sigma}{\pi}}$ & & 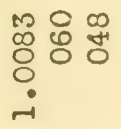 & $\begin{array}{l}m \\
0 \\
0 \\
0\end{array}$ & 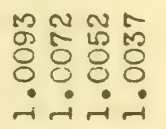 & & 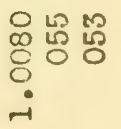 & 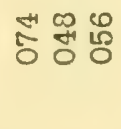 & 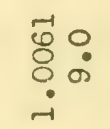 & 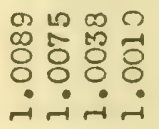 \\
\hline 这 & & 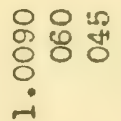 & $\begin{array}{l}-1 \\
80 \\
0 \\
\dot{i}\end{array}$ & $\begin{array}{l}m \\
8 \\
8 \\
8\end{array}$ & & 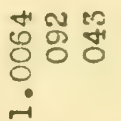 & 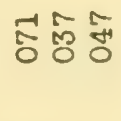 & 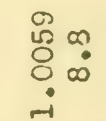 & 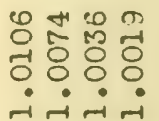 \\
\hline 惌 & & $\begin{array}{l}\text { 응용 } \\
8 \\
0 \\
\text { - }\end{array}$ & : & 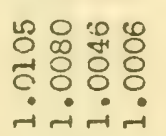 & & 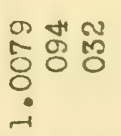 & แี เี็ ำ & 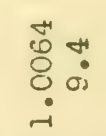 & $\begin{array}{l}\mathscr{0} \\
\mathbb{8} \\
0 \\
0 \\
0 \\
0\end{array}$ \\
\hline ॠँّ & & 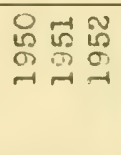 & 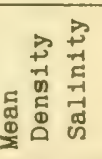 & 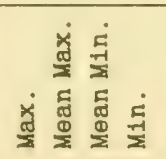 & & 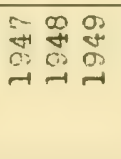 & 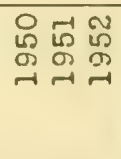 & 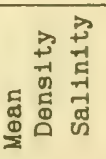 & 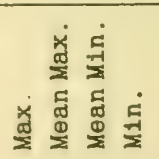 \\
\hline
\end{tabular}




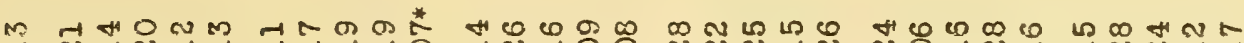

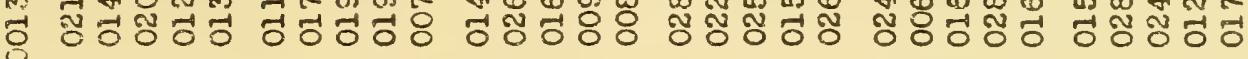
$\stackrel{9}{\circ}$

の 거 -

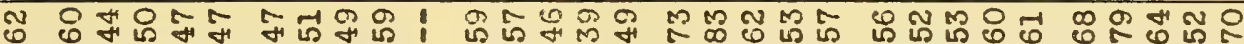

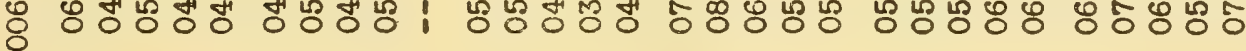

年

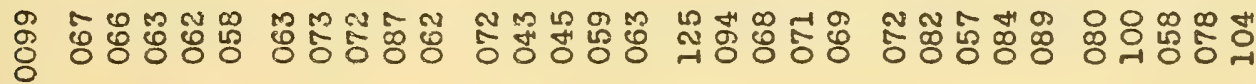

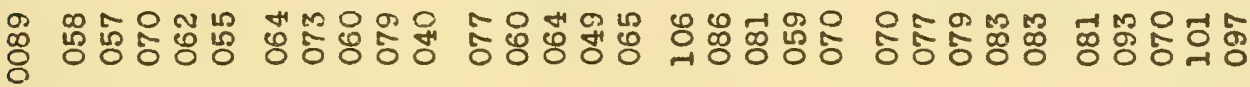

क

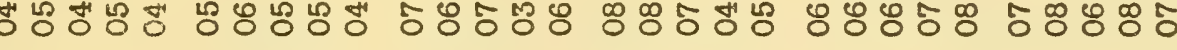

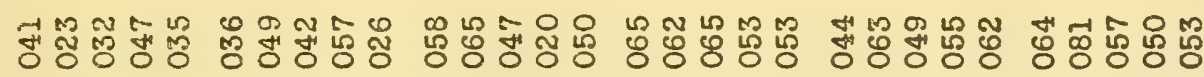

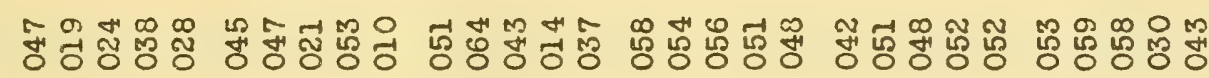

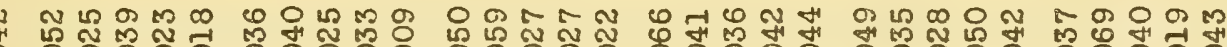

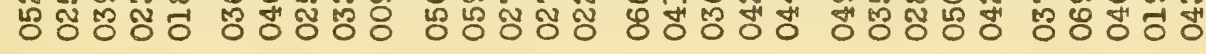

- 6 L

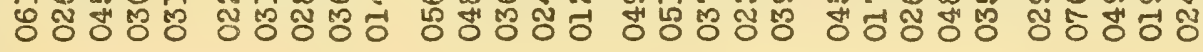

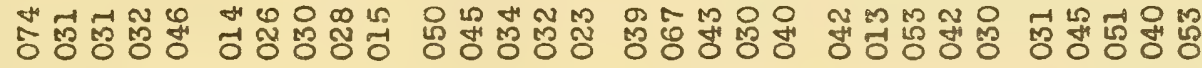

莽

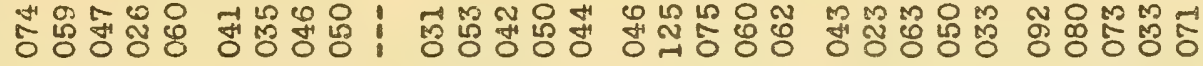

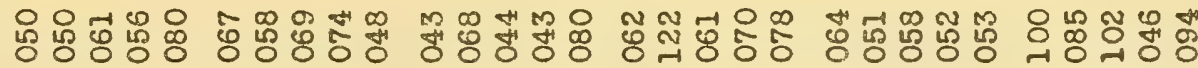

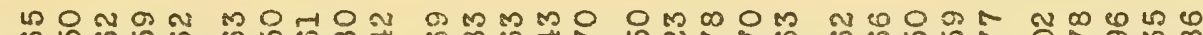

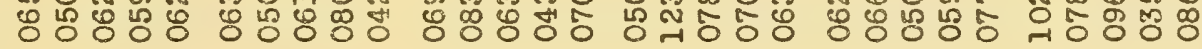




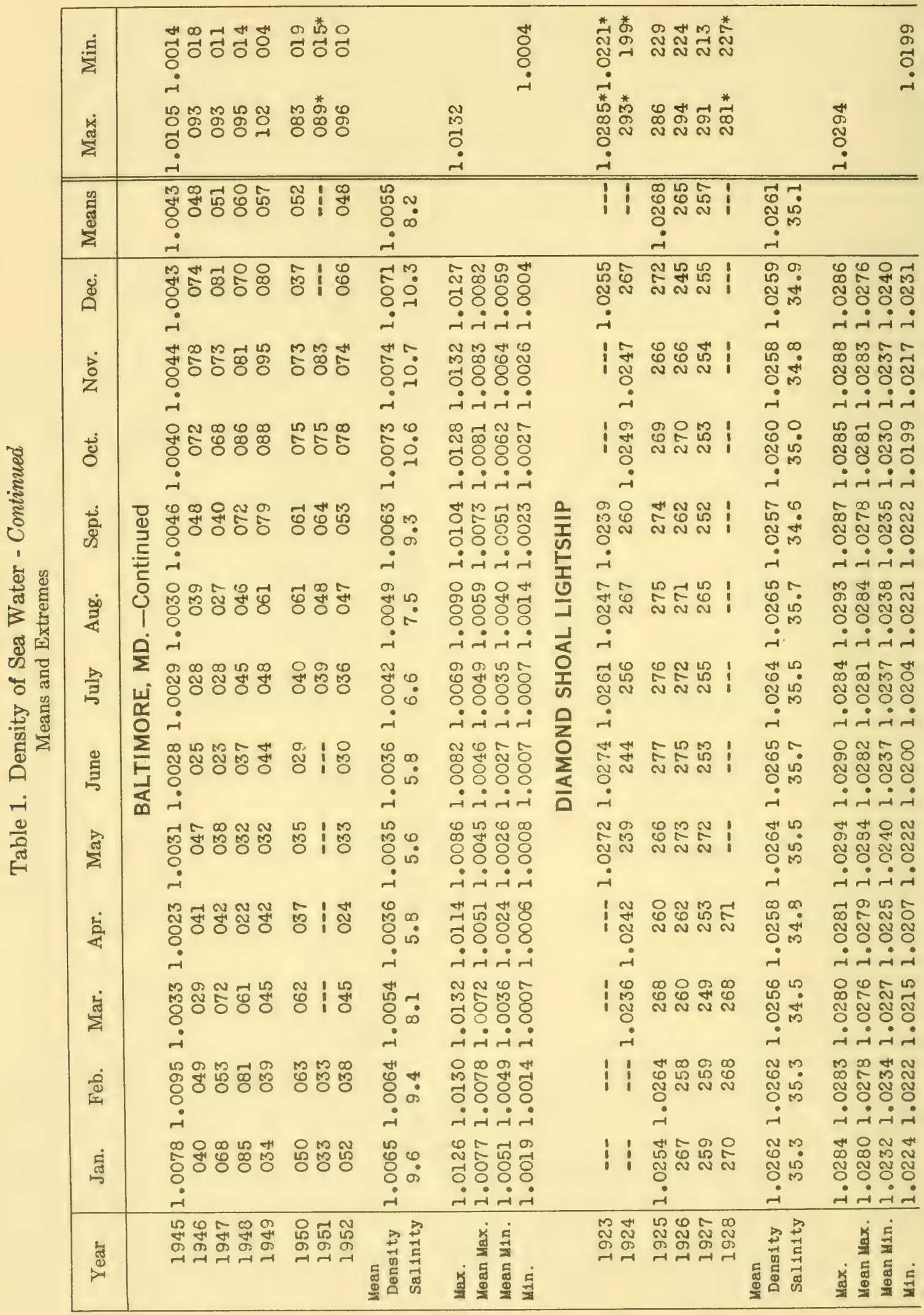




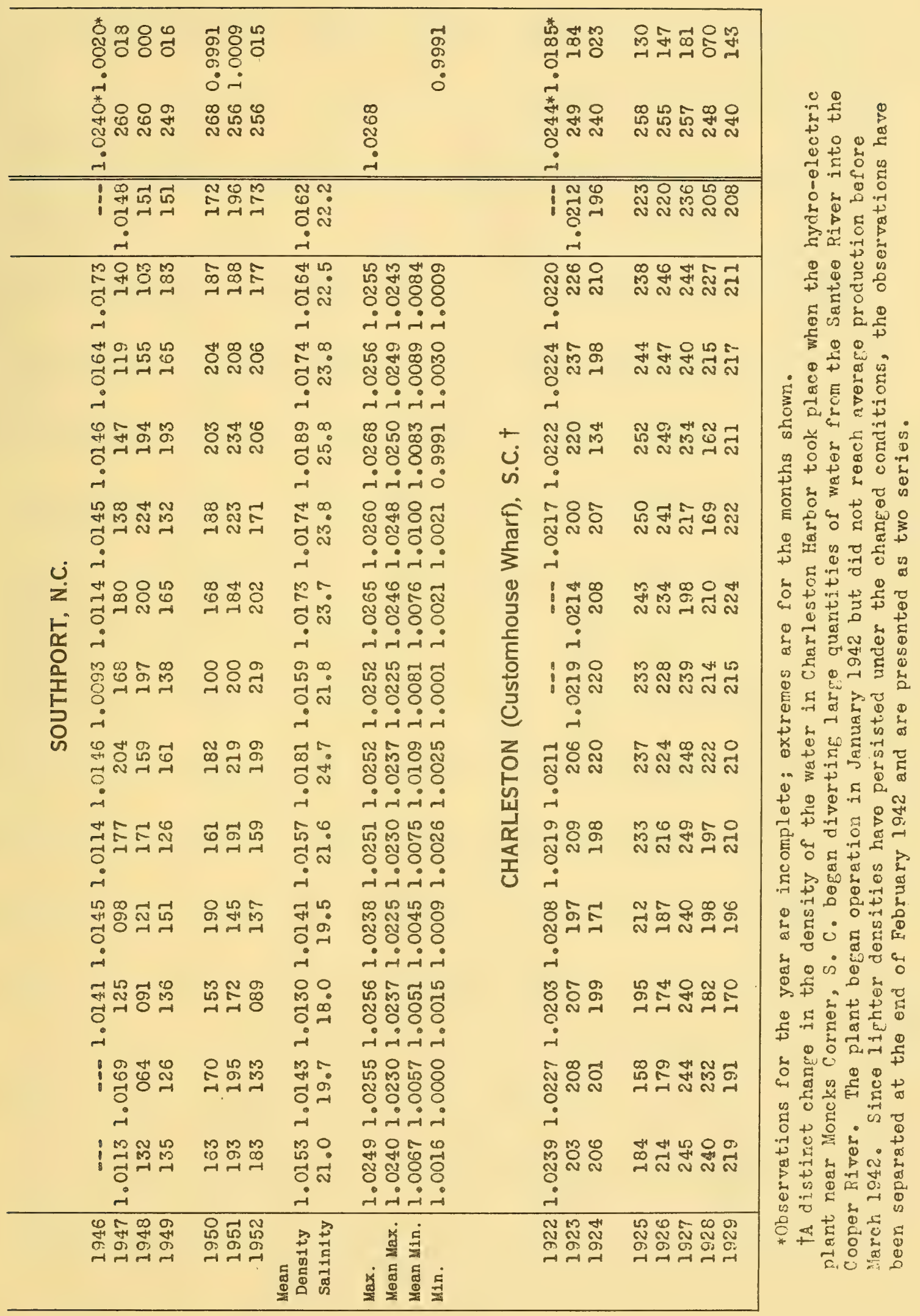




\begin{tabular}{|c|c|c|c|c|c|c|c|c|c|}
\hline$\sum^{\dot{\varpi}}$ & & 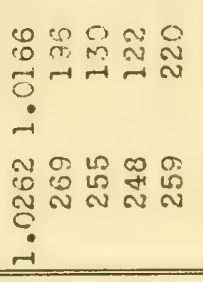 & 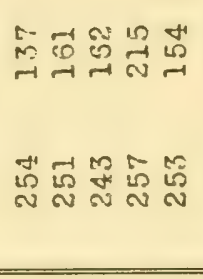 & 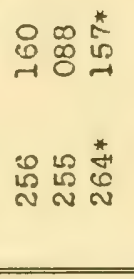 & & $\begin{array}{l}m \\
\text { s } \\
8 \\
0 \\
-1\end{array}$ & & 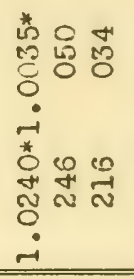 & 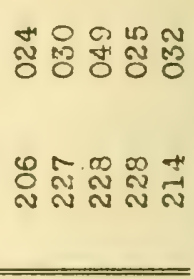 \\
\hline 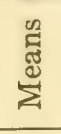 & & 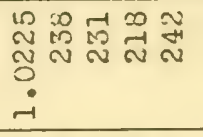 & 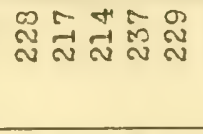 & 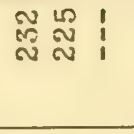 & $\begin{array}{l}0 \\
\text { Ũ } \\
0 \\
0 \\
-1\end{array}$ & & & $\begin{array}{l}-1 \\
0 \\
0 \\
0\end{array}$ & 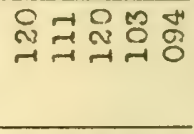 \\
\hline ஜ் & & 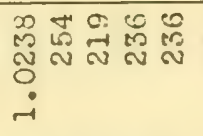 & 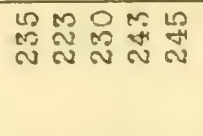 & 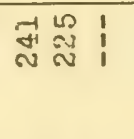 & 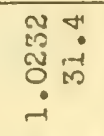 & 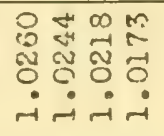 & & 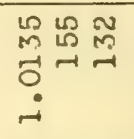 & 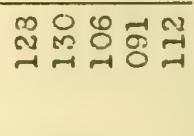 \\
\hline z & 얼 & 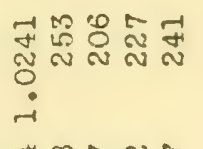 & 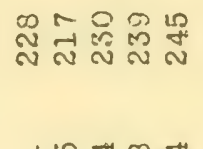 & 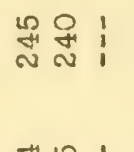 & $\begin{array}{l}\text { लै } \\
\text { गे में } \\
\text { ले }\end{array}$ & 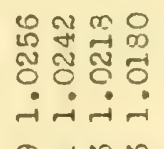 & $\frac{+}{\mathscr{\Phi}}$ & 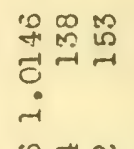 & 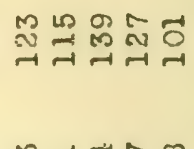 \\
\hline$\dot{0}$ & $\begin{array}{l}\text { 吾 } \\
\text { O } \\
\dot{+}\end{array}$ & 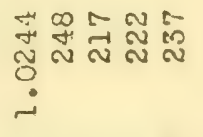 & 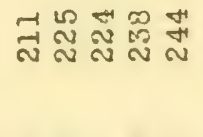 & 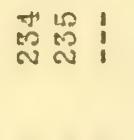 & $\begin{array}{l}m \\
w \\
v \\
0 \\
0 \\
-i\end{array}$ & 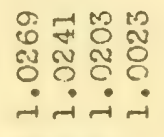 & $\begin{array}{l}0 \\
\text { 음 }\end{array}$ & 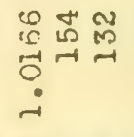 & 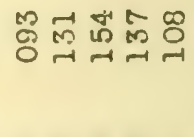 \\
\hline 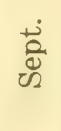 & $\dot{j}$ & 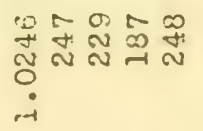 & 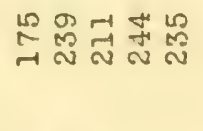 & 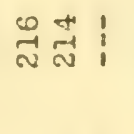 & $\begin{array}{ll}-1 & 0 \\
N & 0 \\
0 & 0 \\
0-1\end{array}$ & 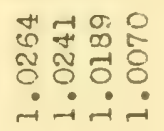 & $\begin{array}{l}\dot{5} \\
\dot{j}\end{array}$ & 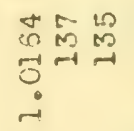 & 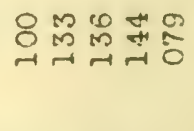 \\
\hline$\sum^{\infty}$ & $\sum_{\substack{\frac{1}{5} \\
\frac{\pi}{5}}}$ & 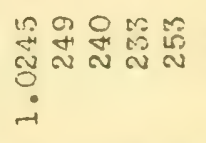 & 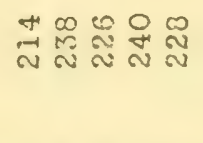 & 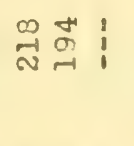 & $\begin{array}{l}\tilde{N} \\
\tilde{N} \\
0 \\
\dot{0}\end{array}$ & 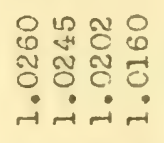 & $\frac{\hat{E}}{\frac{1}{3}}$ & $\begin{array}{lll}\omega & -1 & 0 \\
0 & 0 & -1 \\
0 & -1\end{array}$ & 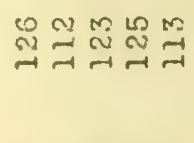 \\
\hline$\frac{2}{3}$ & $\begin{array}{l}\frac{n}{\partial} \\
\text { o } \\
\frac{\varepsilon}{0}\end{array}$ & 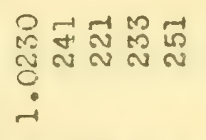 & 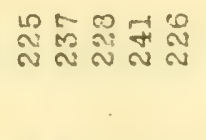 & સ્木 & $\begin{array}{ll}0 & \infty \\
v & \dot{0} \\
\tilde{g} & 0 \\
i & \end{array}$ & 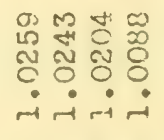 & 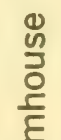 & $\begin{array}{l}0 \\
0 \\
0 \\
0 \\
0\end{array}$ & 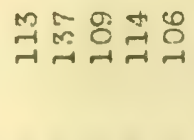 \\
\hline 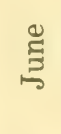 & $\underset{z}{\stackrel{\pi}{3}}$ & 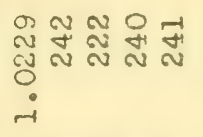 & 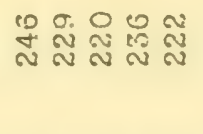 & 苟空! & $\begin{array}{l}\infty \\
N \\
N \\
0 \\
0 \\
-1\end{array}$ & 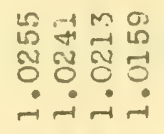 & $\frac{5}{0}$ & $\begin{array}{l}a \\
\overrightarrow{0} \\
\overrightarrow{0} \\
\dot{n}\end{array}$ & $\begin{array}{l}\infty \\
m \\
m\end{array}$ \\
\hline 苾 & 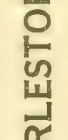 & 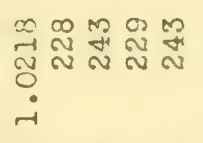 & 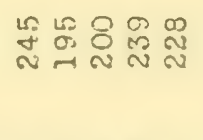 & 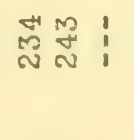 & 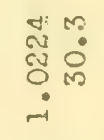 & 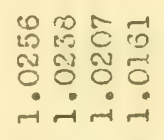 & $\begin{array}{l}z \\
0 \\
\text { w } \\
\text { w }\end{array}$ & 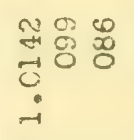 & $\begin{array}{l}\sim \\
\sim\end{array} 50000$ \\
\hline 逢 & $\frac{\sum^{2}}{u}$ & 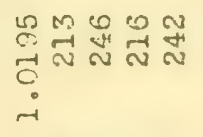 & 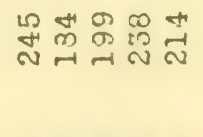 & 心્心 & 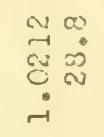 & 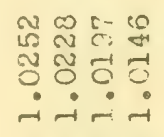 & $\frac{\bar{d}}{\underline{\alpha}}$ & $\begin{array}{lll}\infty & \infty & 1 \\
m & 0 & \infty \\
-1 & 0 & 0 \\
0 & \\
i\end{array}$ & 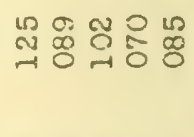 \\
\hline$\sum_{\Sigma}^{\tilde{\sigma}}$ & & 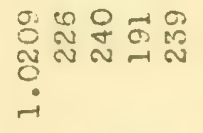 & a & 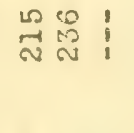 & $\begin{array}{l}\mathscr{D} \\
0 \\
\cup \\
0 \\
0 \\
\sim\end{array}$ & 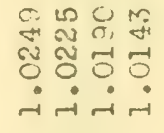 & & 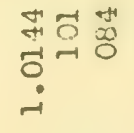 & 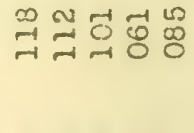 \\
\hline$\dot{0}$ & & 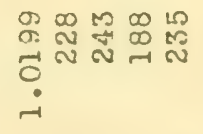 & 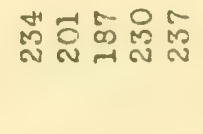 & 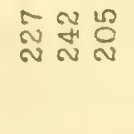 & $\begin{array}{l}+10 \\
-10 \\
0 \\
0 \\
-i\end{array}$ & 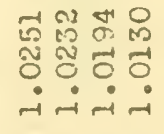 & & i & 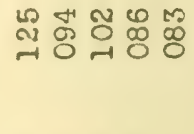 \\
\hline 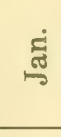 & & 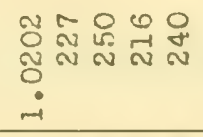 & 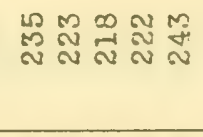 & 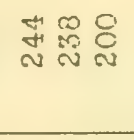 & 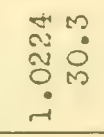 & 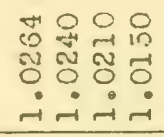 & & is & 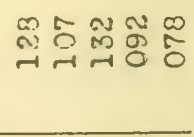 \\
\hline శี & & 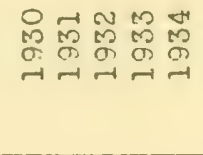 & 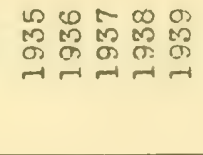 & 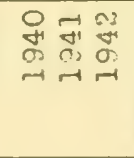 & 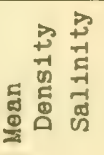 & 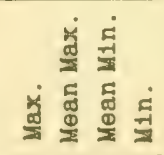 & & 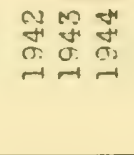 & 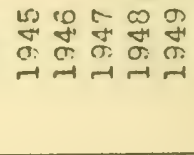 \\
\hline
\end{tabular}




\begin{tabular}{|c|c|c|c|c|c|c|c|c|c|c|c|}
\hline $\begin{array}{l}\infty \\
\text { m }\end{array}$ & & 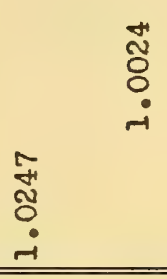 & & 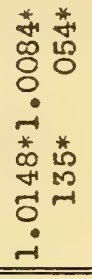 & & 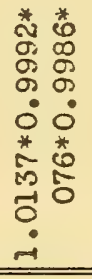 & & 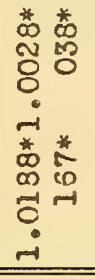 & & 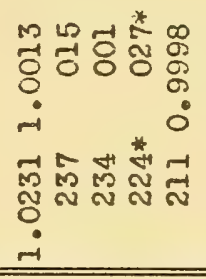 & 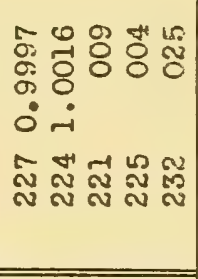 \\
\hline స̃ & $\begin{array}{l}0 \\
0 \\
0 \\
0 \\
0 \\
0\end{array}$ & & & i i & & $i$ & & i i & & 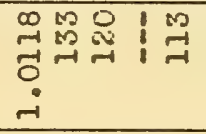 & 湈 \\
\hline 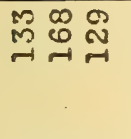 & 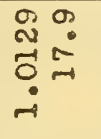 & 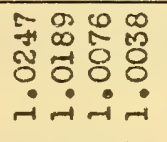 & & $\begin{array}{ll} & 1 \\
N & 1 \\
0 & 1 \\
0 & \\
-1 & \end{array}$ & & \begin{tabular}{l}
0 \\
\hdashline \\
8 \\
8 \\
0 \\
-1
\end{tabular} & $\dot{j}$ & $\begin{array}{l}0 \\
\stackrel{5}{0} ! \\
0 \\
0 \\
-1\end{array}$ & & 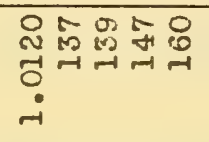 & 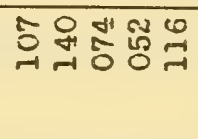 \\
\hline 点禺 & $\begin{array}{l}\infty \\
0 \\
m \\
0 \\
0 \\
0\end{array}$ & 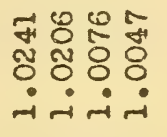 & & $\begin{array}{l}\infty \\
m \\
-1 \\
0 \\
-1\end{array}$ & $\dot{j}$ & $\begin{array}{l}5 \\
\text { is ! } \\
8 \\
0 \\
-i\end{array}$ & 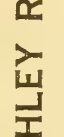 & 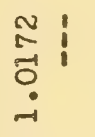 & & 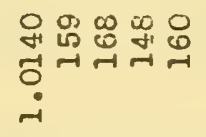 & मून \\
\hline 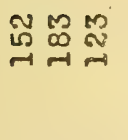 & 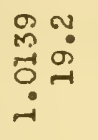 & 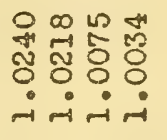 & & 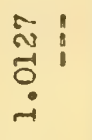 & $\sum$ & $\begin{array}{c}0 \\
1 \\
0 \\
8 \\
0 \\
-1\end{array}$ & $\frac{\bar{\sigma}}{\frac{1}{0}}$ & $\begin{array}{l}\mathfrak{m} \\
0 \\
\stackrel{1}{0} \\
\vdots \\
-1\end{array}$ & ভ் & 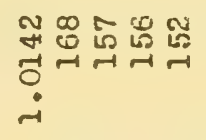 & 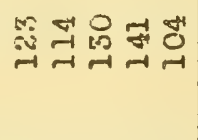 \\
\hline 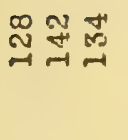 & $\begin{array}{l}0 \\
m \\
0 \\
0 \\
0\end{array}$ & 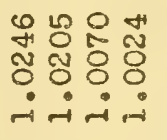 & ن & $\begin{array}{l}8 \\
8 \\
-1 \\
0 \\
0 \\
-1\end{array}$ & $\begin{array}{l}\text { u } \\
0 \\
0\end{array}$ & $\begin{array}{l}\infty \\
\stackrel{\infty}{1} \\
\stackrel{8}{0} \\
-1\end{array}$ & $\sum_{j}^{5}$ & $\begin{array}{l}0 \\
m \\
m \\
0 \\
0 \\
-1\end{array}$ & $\frac{\infty}{a}$ & 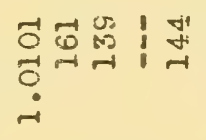 & 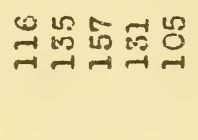 \\
\hline $\begin{array}{l}0 \\
0\end{array}$ & $\begin{array}{c}-10 \\
-1 \\
0 \\
0\end{array}$ & 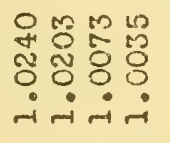 & $\begin{array}{l}\geq \\
0 \\
0\end{array}$ & $\begin{array}{l}-1 \\
8 \\
8 \\
-1\end{array}$ & $\sum_{1}^{\infty}$ & $\begin{array}{l}0 \\
\stackrel{0}{8} \\
- \\
-1\end{array}$ & . & $\begin{array}{ll}\hat{N} & 1 \\
0 & 1 \\
0 & \end{array}$ & $\sum_{\frac{1}{z}}^{\frac{1}{z}}$ & 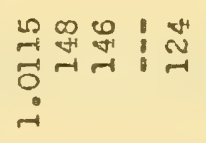 & 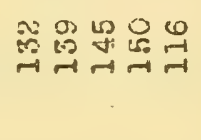 \\
\hline 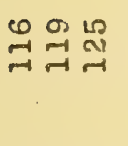 & $\begin{array}{l}c \\
\stackrel{0}{0} \\
0 \\
-1\end{array}$ & 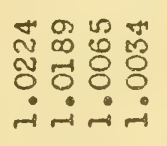 & $\sum_{2}^{2}$ & $\begin{array}{l}2 \\
0 \\
8 \\
8 \\
-1\end{array}$ & $\frac{\Sigma}{\alpha}$ & 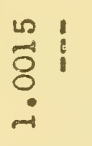 & 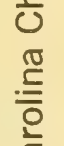 & 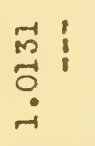 & $\frac{\pi}{\sqrt{x}}$ & $\begin{array}{l}\infty \\
m \\
\infty \\
0 \\
0 \\
0\end{array}$ & 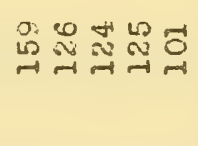 \\
\hline 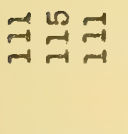 & 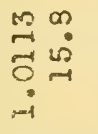 & 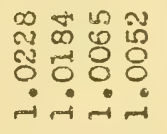 & $\frac{0}{2}$ & $\begin{array}{ll}\text { L } & 1 \\
0 & 1 \\
0 & 1 \\
0 & 1\end{array}$ & $\begin{array}{l}0 \\
\text { un } \\
\end{array}$ & $\begin{array}{l}5 \\
\delta \\
8 \\
\dot{0}\end{array}$ & $\begin{array}{l}\pi \\
\dot{0} \\
\frac{\pi}{E}\end{array}$ & $\begin{array}{l}4 \\
0 \\
0 \\
0 \\
0 \\
-1\end{array}$ & $\sum_{2}^{0}$ & 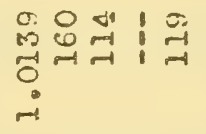 & 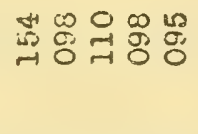 \\
\hline 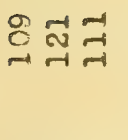 & $\underbrace{+\infty}_{-1}$ & 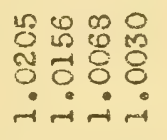 & $\mathcal{U}$ & $\begin{array}{l}410 \\
0: 0 \\
-10 \\
0 \\
0\end{array}$ & $\frac{\pi}{\frac{\pi}{x}}$ & $i \frac{0}{8}$ & $\sum_{z}^{\frac{\bar{L}}{20}}$ & 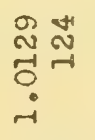 & 贷 & 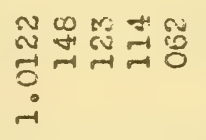 & 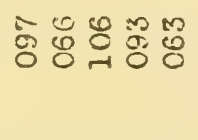 \\
\hline 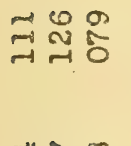 & $\begin{array}{l}0 \\
0 \\
0 \\
0\end{array}$ & 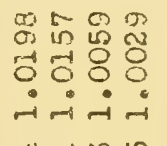 & & $\begin{array}{l}4 \\
0 \\
0 \\
0 \\
1 \\
1\end{array}$ & $\begin{array}{l}\frac{1}{10} \\
\frac{\alpha}{2}\end{array}$ & 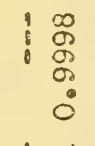 & $\begin{array}{l}0 \\
\text { w } \\
\text { w } \\
\frac{1}{\alpha}\end{array}$ & $\begin{array}{l}0 \\
1 \\
1 \\
0 \\
8 \\
0 \\
-1\end{array}$ & & $\begin{array}{l}\infty \\
0 \\
0 \\
0 \\
0 \\
0 \\
-1\end{array}$ & 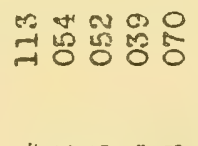 \\
\hline 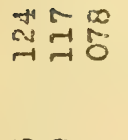 & $\begin{array}{l}0 \\
0 \\
0 \\
-1\end{array}$ & 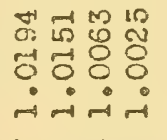 & & $\begin{array}{l}\not \\
0 \\
8 \\
0 \\
0 \\
1\end{array}$ & & 15 & $\frac{1}{0}$ & $\begin{array}{l}120 \\
10 \\
0 \\
0 \\
-1\end{array}$ & & $\begin{array}{l}m \\
0 \\
0 \\
0 \\
0 \\
0 \\
0\end{array}$ & 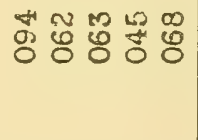 \\
\hline 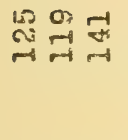 & $\begin{array}{l}0 \\
-1 \\
0 \\
0 \\
0\end{array}$ & 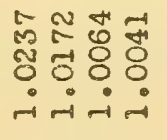 & & $\frac{n}{9}$ & & i & & $\begin{array}{l}1 \\
1 \\
i-1 \\
0 \\
0 \\
-1\end{array}$ & & 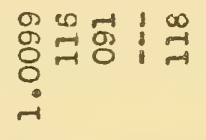 & 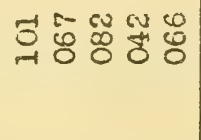 \\
\hline 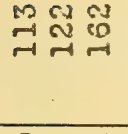 & $\begin{array}{l}0 \\
\text { N1 } \\
0 \\
0 \\
0 \\
-1\end{array}$ & 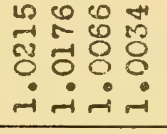 & & 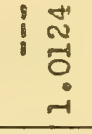 & & $\begin{array}{l}1 \\
1 \\
0 \\
0 \\
0 \\
- \\
\end{array}$ & & $\begin{array}{l}0 \\
0 \\
1 \\
0 \\
0 \\
0\end{array}$ & & 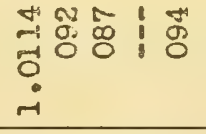 & 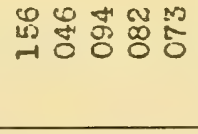 \\
\hline 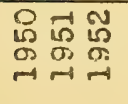 & & & & 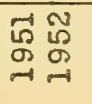 & & 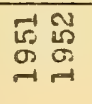 & & 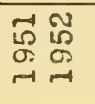 & & 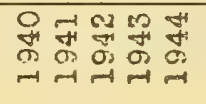 & 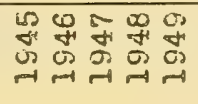 \\
\hline
\end{tabular}




\begin{tabular}{|c|c|c|c|c|c|c|c|c|c|c|}
\hline 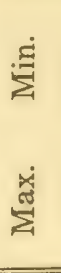 & & 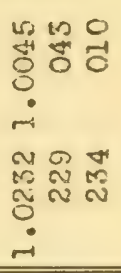 & & $\begin{array}{l}\tilde{M} \\
\stackrel{0}{U} \\
\stackrel{0}{0}\end{array}$ & & $\begin{array}{l}1 \\
6 \\
0 \\
0 \\
0 \\
-1 \\
0 \\
0 \\
+1 \\
0 \\
0 \\
-1 \\
\end{array}$ & 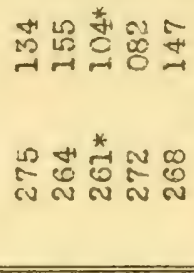 & 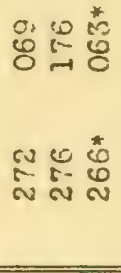 & & $\begin{array}{c}0 \\
0 \\
0 \\
0 \\
0 \\
0\end{array}$ \\
\hline$\sum_{\Sigma}^{\mathscr{J}}$ & & 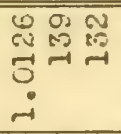 & $\begin{array}{l}\omega \\
-1 \\
-1 \\
0 \\
0 \\
-1\end{array}$ & & & ! & 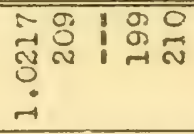 & 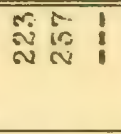 & 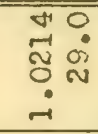 & \\
\hline ه் & & 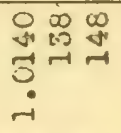 & 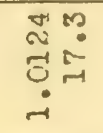 & 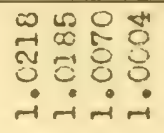 & & $\begin{array}{l}0 \\
\stackrel{1}{\pi} \\
\stackrel{c}{c} \\
-1\end{array}$ & 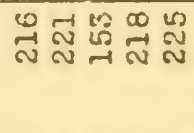 & 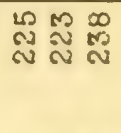 & 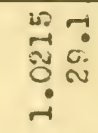 & 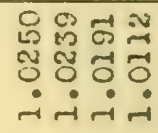 \\
\hline 客 & ర్ల & $\begin{array}{lll}0 & 0 & 0 \\
0 & 0 & 0 \\
-1 & -1 & -1 \\
0 & -1\end{array}$ & $\begin{array}{l}-0 \\
\text { in } \\
\text { - } \\
0 \\
-1\end{array}$ & 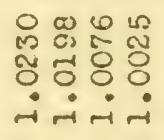 & & $\begin{array}{l}10 \\
\text { E } \\
\vdots \\
0 \\
-1\end{array}$ & 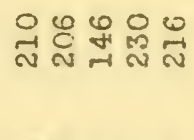 & Љָ & 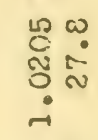 & 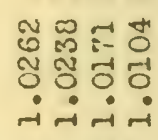 \\
\hline ث் & 亮 & 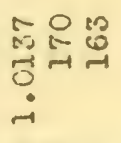 & $\begin{array}{l}2 \\
-1 \\
0 \\
0\end{array}$ & 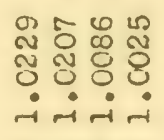 & & $\begin{array}{l}1 \\
\infty \\
0 \\
0 \\
0 \\
-1\end{array}$ & 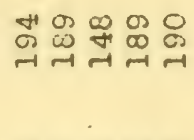 & 点 & $\begin{array}{l}\sigma_{0}^{0} \\
\overrightarrow{0}: 0 \\
\dot{-i}\end{array}$ & 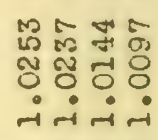 \\
\hline $\begin{array}{l}\text { 密 } \\
\text { के }\end{array}$ & : & 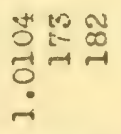 & 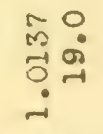 & 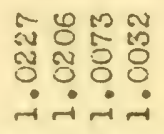 & $<\dot{<}$ & $\begin{array}{c}\infty \\
\infty \\
0 \\
0 \\
-1\end{array}$ & 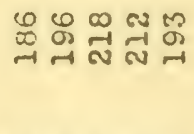 & w芯 & 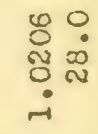 & 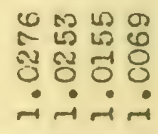 \\
\hline$\sum_{3}^{\infty}$ & $\sum_{I}^{\frac{1}{I I}}$ & $\begin{array}{lll}0 & 2 \\
0 & 6 & 5 \\
-1 & -1 & -1 \\
0 & -1\end{array}$ & 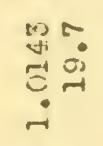 & 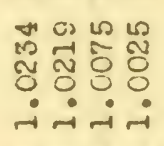 & $\frac{1}{2}$ & $\begin{array}{l}5 \\
5 \\
5 \\
-1\end{array}$ & 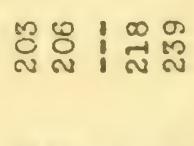 & 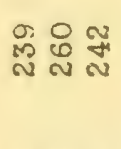 & 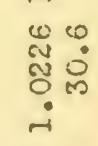 & 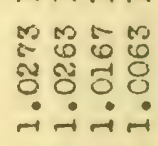 \\
\hline$\frac{2}{3}$ & $\frac{1}{\sum}$ & 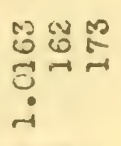 & 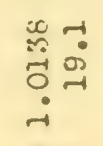 & 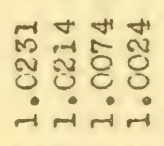 & $\sum_{\substack{\alpha \\
\alpha}}$ & 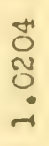 & 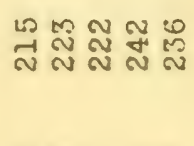 & 岱 & 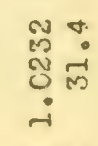 & 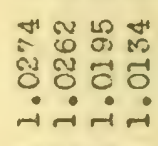 \\
\hline$\underset{\Xi}{\Xi}$ & $\begin{array}{l}\text { w } \\
\frac{x}{w}\end{array}$ & 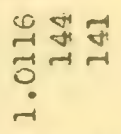 & $\begin{array}{l}\dot{m} \\
\stackrel{-1}{0} \\
-1\end{array}$ & 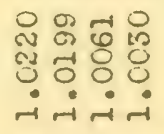 & 岀 & $\begin{array}{l}0 . \\
\stackrel{-1}{~} \\
0 \\
-1\end{array}$ & 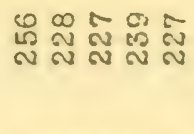 & 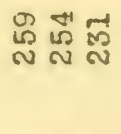 & $\begin{array}{l}\infty \\
\tilde{n} \\
\tilde{\sigma} \\
\dot{\sim}\end{array}$ & 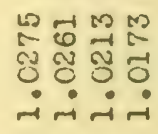 \\
\hline 离 & $\sum_{2}^{\frac{\pi}{2}}$ & $\begin{array}{l}\infty \\
0 \\
0 \\
0\end{array}$ & $\begin{array}{l}\overrightarrow{0} \\
\dot{0} \\
\dot{0} \\
\dot{-1}\end{array}$ & 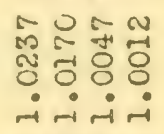 & & i & 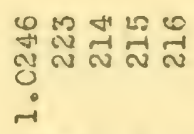 & 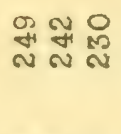 & 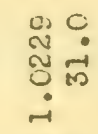 & 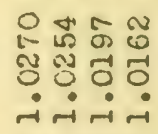 \\
\hline$\dot{\mathrm{a}}$ & 点 & 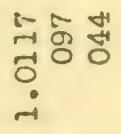 & ${ }^{5}-1$ & 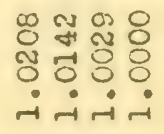 & & í & 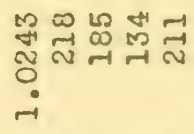 & 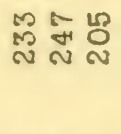 & 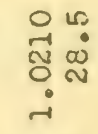 & 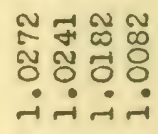 \\
\hline$\sum_{\Sigma}^{\infty}$ & & 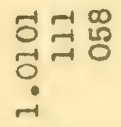 & 둥 & 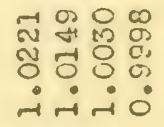 & & i & 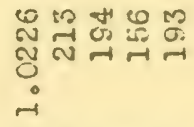 & స్心 & 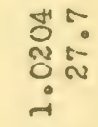 & 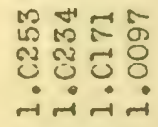 \\
\hline 远 & & 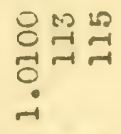 & $\begin{array}{l}0 \\
\sigma \\
0 \\
0 \\
0 \\
i\end{array}$ & 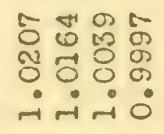 & & i & 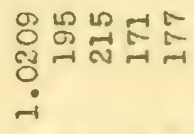 & $\begin{array}{ll}\text { mo : } & 1 \\
\text { N } & \text { in }\end{array}$ & 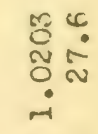 & 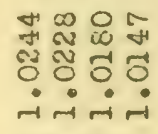 \\
\hline 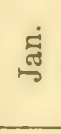 & & 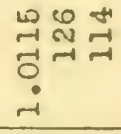 & $\begin{array}{ll}0 . & 0 \\
0 & 0 \\
0 & -1 \\
0 & -1 \\
-1\end{array}$ & 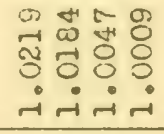 & & i & 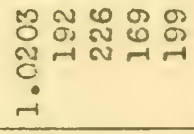 & $\begin{array}{l}\infty \stackrel{0}{\sim} \underset{N}{\sim} \\
\stackrel{N}{\sim} \underset{N}{N}\end{array}$ & $\begin{array}{l}5 \% \\
0 \\
0 \\
0 \\
\end{array}$ & 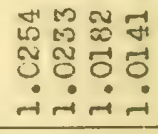 \\
\hline 㞼 & & 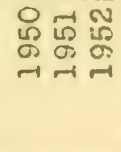 & 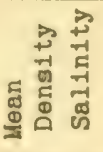 & 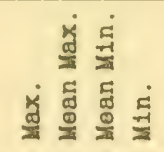 & & $\underset{+}{\stackrel{+}{c}}$ & 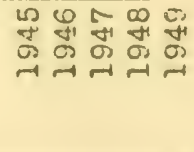 & 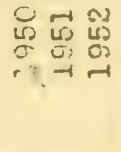 & 党 & 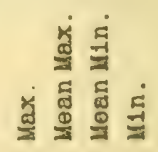 \\
\hline
\end{tabular}




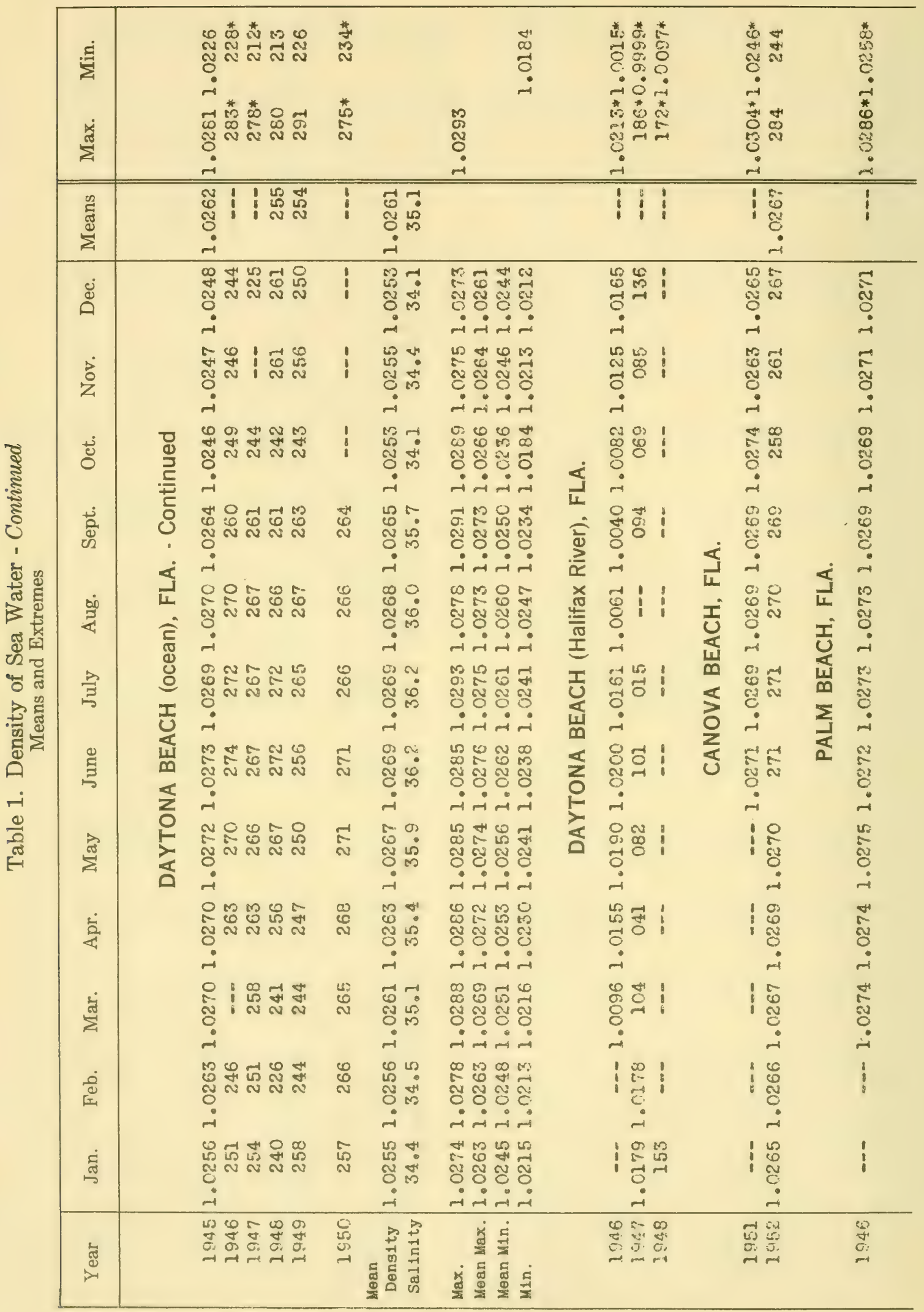




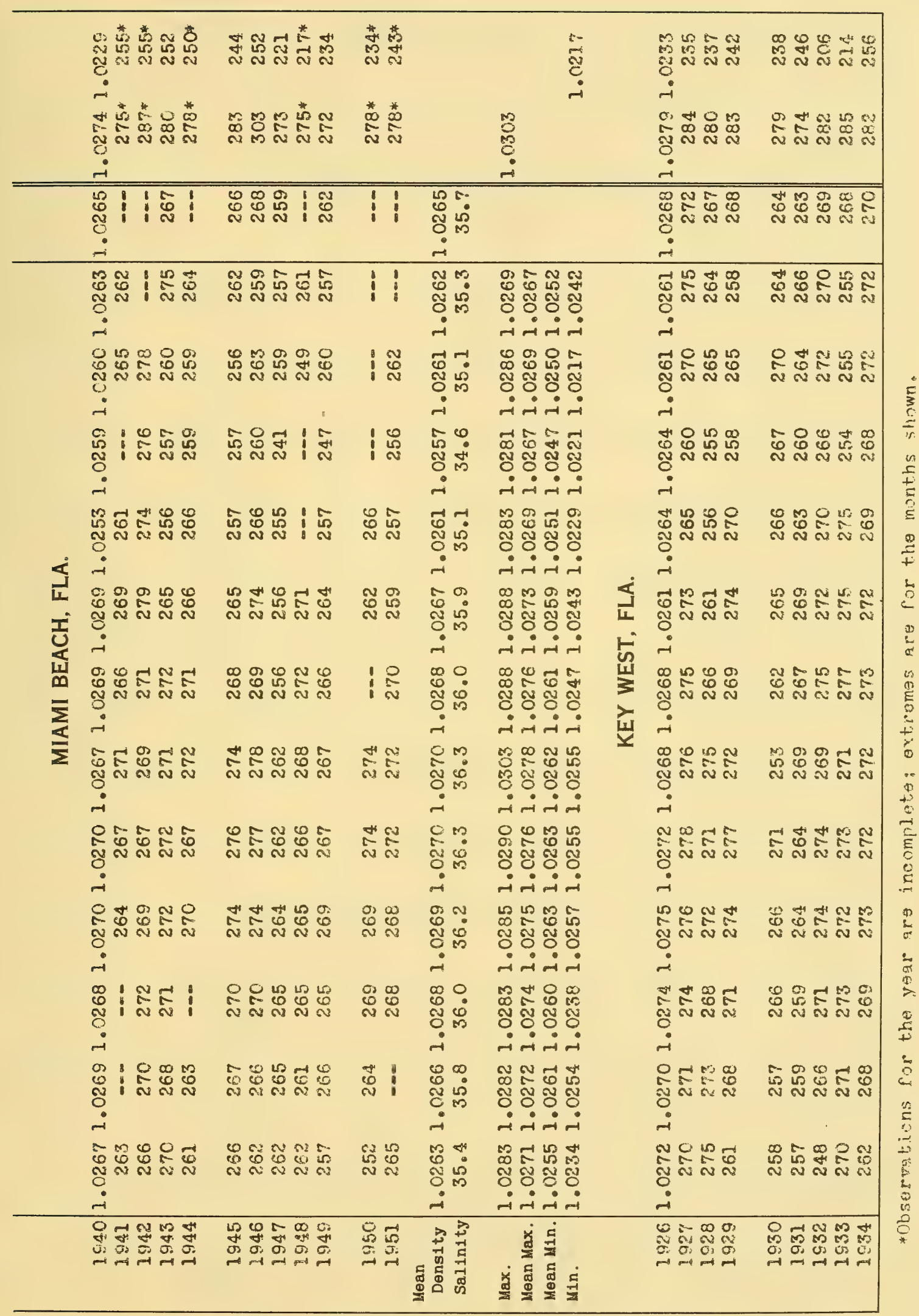




\begin{tabular}{|c|c|c|c|c|c|c|c|c|c|}
\hline 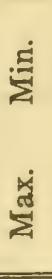 & & 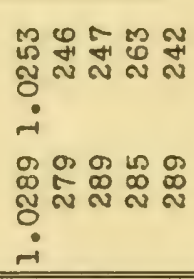 & $\begin{array}{l}\text { * } \\
\stackrel{\infty}{\infty} \\
\text { 心 }\end{array}$ & 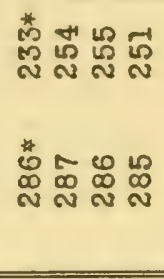 & 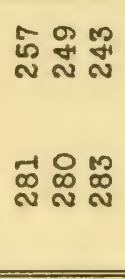 & & $\begin{array}{l}0 \\
0 \\
\tilde{~} \\
:\end{array}$ & & 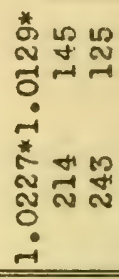 \\
\hline 宽 & & 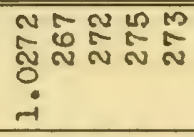 & ! & ! ָㅗ & 온 & $\begin{array}{l}0 \\
\text { กิ } \\
0 \\
0\end{array}$ & & & $\begin{array}{l}-1 \\
1 \\
0 \\
0 \\
0 \\
-1\end{array}$ \\
\hline ஜ் & & 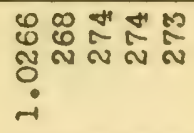 & ! & 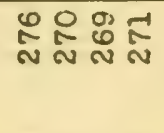 & ผ & $\begin{array}{l}0 \\
\mathscr{N} \\
N \\
0 \\
0 \\
-1\end{array}$ & 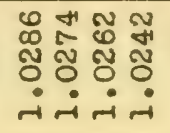 & & 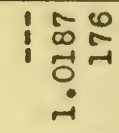 \\
\hline 官 & & 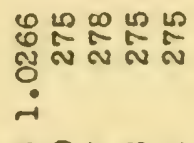 & i & 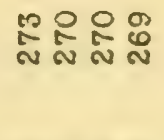 & 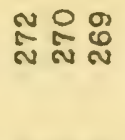 & 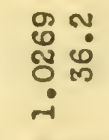 & 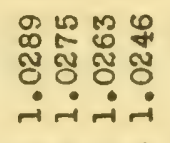 & & $\begin{array}{l}1 \\
1 \\
1 \\
0 \\
0 \\
0 \\
-1\end{array}$ \\
\hline ப் & & 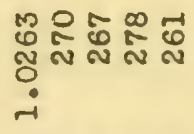 & $\stackrel{\circ}{\text { ก }}$ & 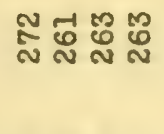 & 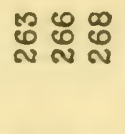 & 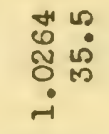 & 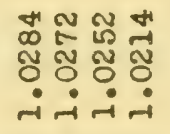 & & $\begin{array}{l}1 \\
1 \\
1 \\
0 \\
0 \\
0 \\
j\end{array}$ \\
\hline थूँ & 일 & 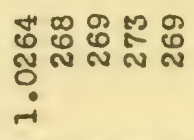 & : & 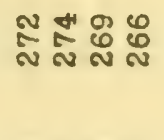 & 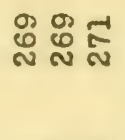 & 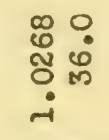 & 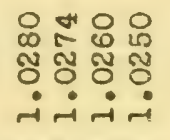 & $\dot{x}$ & 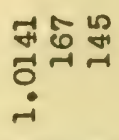 \\
\hline 蛋 & 풍 & 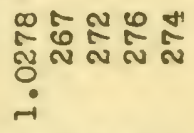 & $\underset{N}{\stackrel{N}{N}}$ & 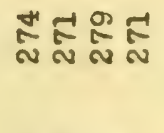 & 온융 & 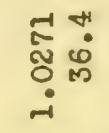 & 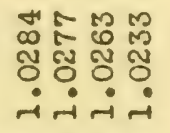 & $\underbrace{0}_{\infty}$ & 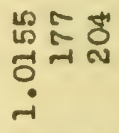 \\
\hline$\frac{2}{3}$ & 노 & 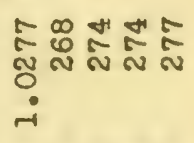 & $\begin{array}{l}\text { O } \\
\text { 品 } \\
\text { d }\end{array}$ & 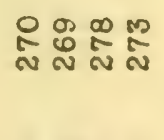 & พิ듀 & 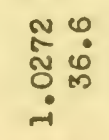 & 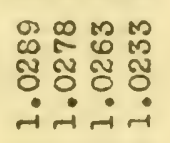 & 点 & 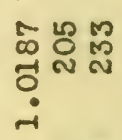 \\
\hline$\stackrel{\Xi}{3}$ & 崩 & 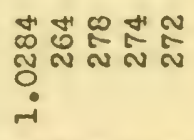 & ᄋ్ & 念志怘令 & 촌온온 & 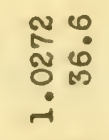 & 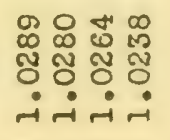 & $\stackrel{2}{5}$ & 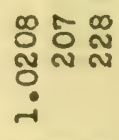 \\
\hline$\stackrel{\text { }}{\Sigma}$ & & 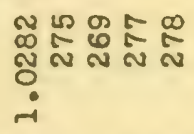 & $\stackrel{\infty}{\infty}$ & 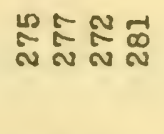 & ํㅗㄴ & 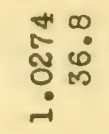 & 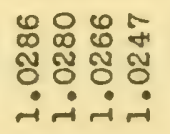 & & 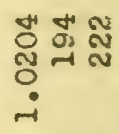 \\
\hline 客 & & 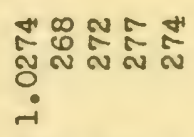 & 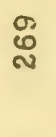 & โ็ำ & 온 & $\begin{array}{l}m \\
5 \\
0 \\
0 \\
0\end{array}$ & 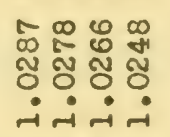 & & 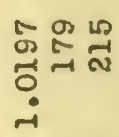 \\
\hline$\sum_{\Sigma}^{\infty}$ & & 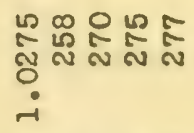 & 온 & 点芯选令 & $\underset{N}{\sim} \stackrel{\infty}{\sim} \underset{N}{\mathbb{N}}$ & $\begin{array}{l}E \\
E \\
0\end{array}$ & 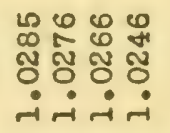 & & 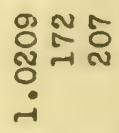 \\
\hline 远 & & 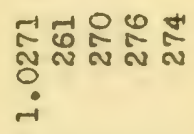 & $\begin{array}{l}\infty \\
\vdots \\
心\end{array}$ & ! & స్ & $\begin{array}{ll}\infty & 0 \\
0 & \bullet \\
\text { O } & 0 \\
:-1 & \end{array}$ & 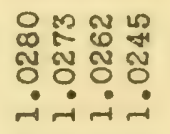 & & 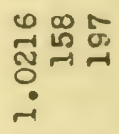 \\
\hline હ્ં & & 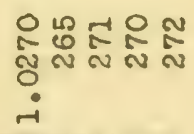 & $\stackrel{0}{\omega}$ & !ลัง & 온온 & $\begin{array}{ll}\infty & 0 \\
0 & 0 \\
\sim & 0 \\
0 & m \\
i & \end{array}$ & 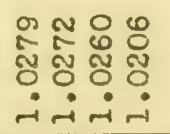 & & 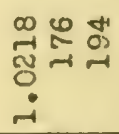 \\
\hline 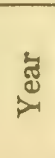 & & 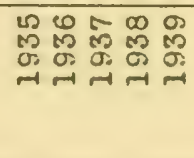 & 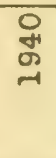 & 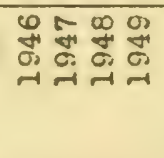 & 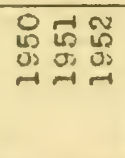 & 索志 & 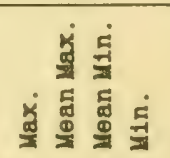 & & 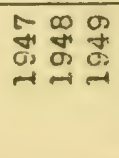 \\
\hline
\end{tabular}




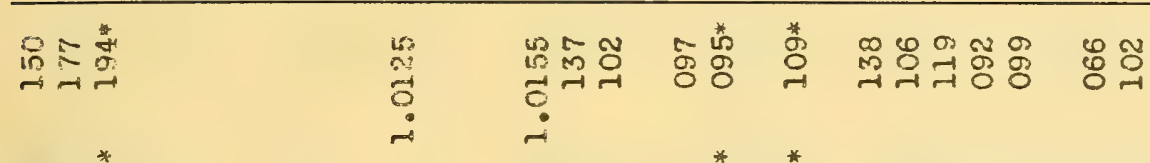

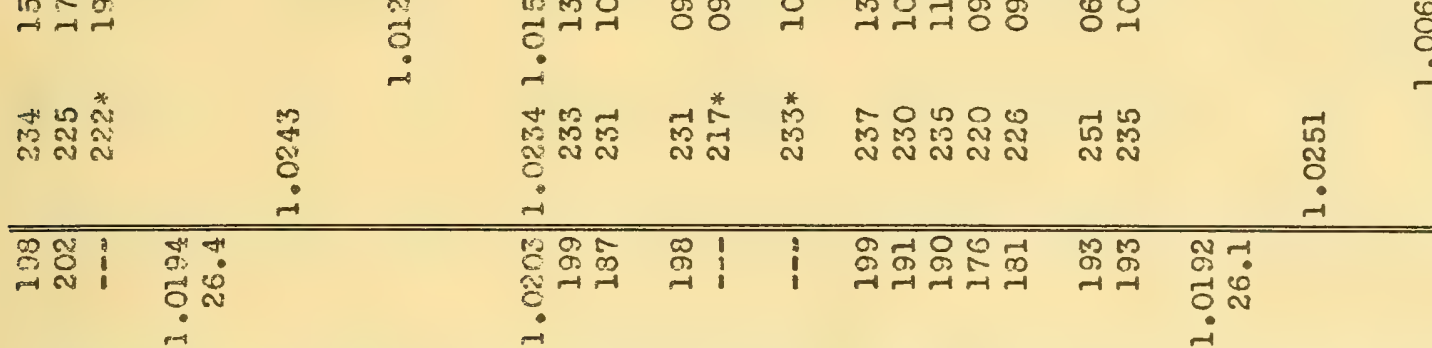

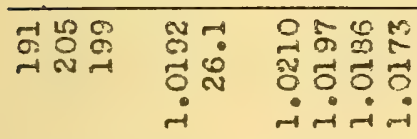

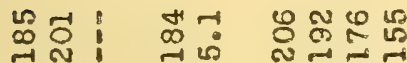

भก त్ర -i $\quad$ ini $i$

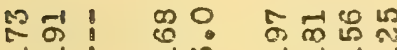

개 ठै $\rightarrow$ तi $\rightarrow$ -

mine mona

-

- 000 ก

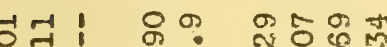

ำ

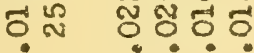

$\rightarrow$

in-1 $-1-1$

我

สิ

- $i$.

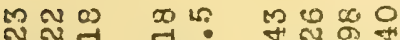

สัง ญี

$\dot{\sim} \quad \dot{-}-\dot{-} \dot{-}$

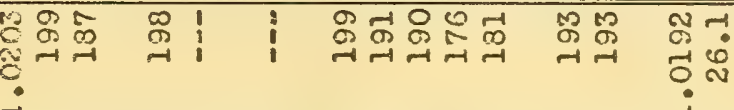

$\rightarrow-1$

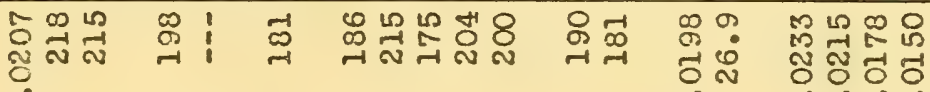
$-1$

б요 के ปูก N $N$ N N mi

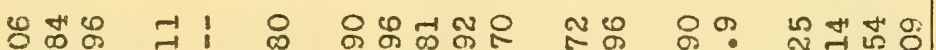

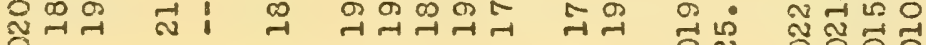
-

चन त त तै

. $\rightarrow$

분요

ठีन-

$\rightarrow$

a) 0

क क

of

$\rightarrow$

赵空

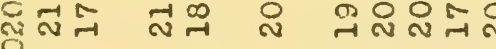

aid

تేڤ

ชิ่

$\therefore$

성요

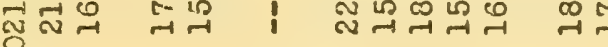

$\dot{\square}$

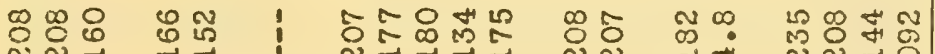

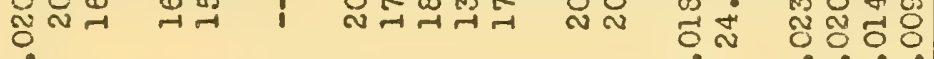
-

\section{H} $\rightarrow$

-i $\dot{-1}-\dot{ }$

o $12 \pi$ 的

స्ल -

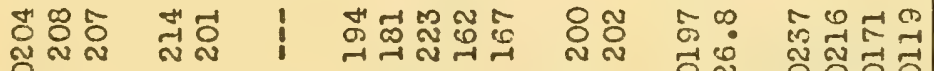
ชूN NN न $-$ $-4$ -1
-1

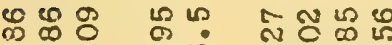

न

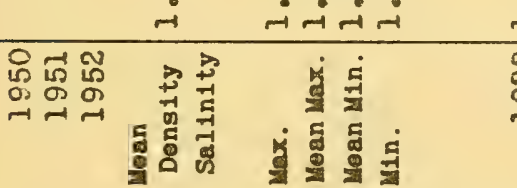

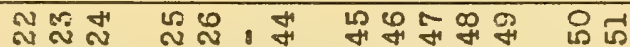

के क्न 


\begin{tabular}{|c|c|c|c|c|c|c|c|}
\hline$\underset{\dot{\varpi}}{\dot{\varpi}}$ & & 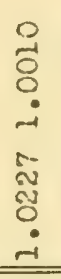 & 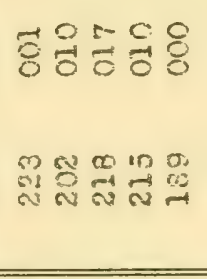 & 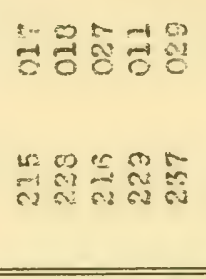 & 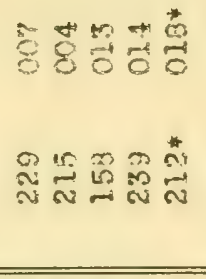 & 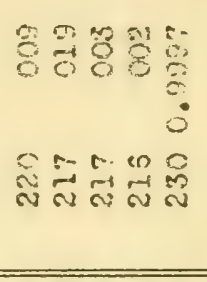 & 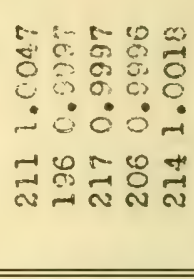 \\
\hline 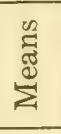 & & $\begin{array}{r}4 \\
0 \\
0 \\
0 \\
-1\end{array}$ & $\begin{array}{l}-10 \\
\cdots\end{array}$ & 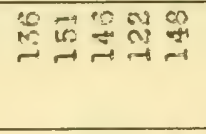 & 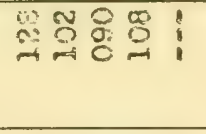 & 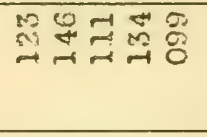 & 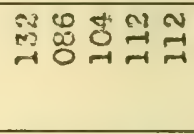 \\
\hline ه્d & & $\begin{array}{l}\hat{E} \\
\overrightarrow{0} \\
-i\end{array}$ & 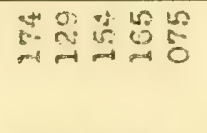 & 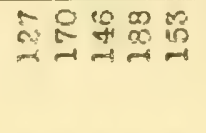 & 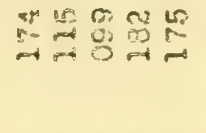 & 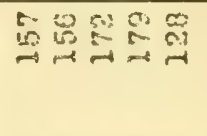 & 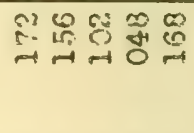 \\
\hline z & & $\begin{array}{l}\infty \\
\stackrel{5}{0} \\
\dot{0} \\
-1\end{array}$ & $b_{-1}^{2}+5$ & 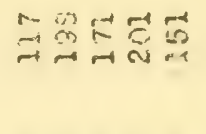 & के & 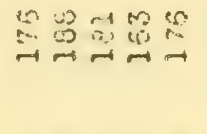 & 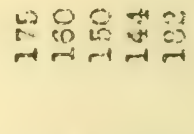 \\
\hline ن் & & $\begin{array}{l}0 \\
0 \\
0 \\
-1\end{array}$ & $\delta^{8}=-$ & 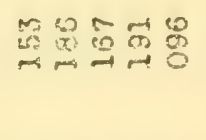 & 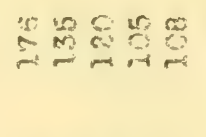 & 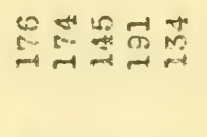 & EN \\
\hline $\begin{array}{l}\text { 夰 } \\
\text { कू }\end{array}$ & & $\begin{array}{l}0 \\
0 \\
0 \\
-1\end{array}$ & 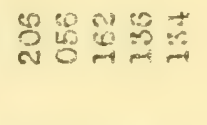 & mis & 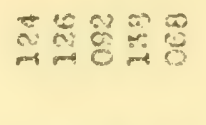 & 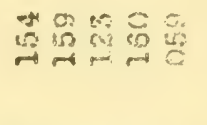 & 虫 \\
\hline 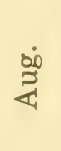 & $\frac{\dot{4}}{5}$ & $\stackrel{\sim}{\stackrel{-1}{0}}$ & $\Rightarrow 0=0$ & 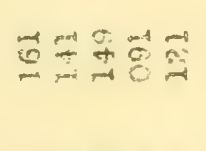 & 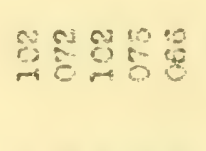 & 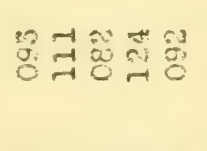 & 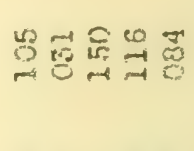 \\
\hline$\frac{2}{3}$ & $\begin{array}{l}0 \\
\text { un } \\
\text { w }\end{array}$ & $\begin{array}{l}\text { के } \\
8 \\
0 \\
\text { - }\end{array}$ & 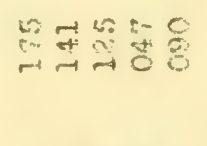 & 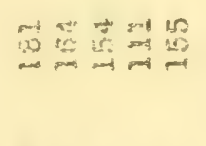 & 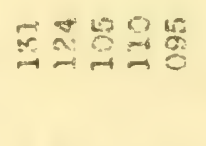 & 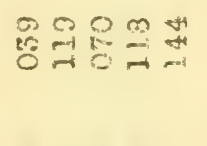 & 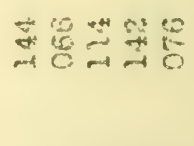 \\
\hline$\stackrel{\varpi}{\Xi}$ & $\bar{a}$ & $\begin{array}{l}\infty \\
\infty \\
8\end{array}$ & 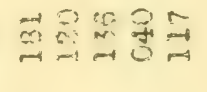 & 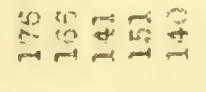 & 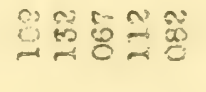 & 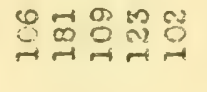 & 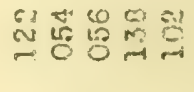 \\
\hline & & $\dot{\sim}$ & & & & & \\
\hline$\sum_{i}^{\infty}$ & & $\begin{array}{c}\ddot{0} \\
\text { О! }\end{array}$ & 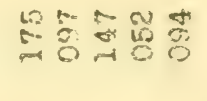 & in & \&. & $\begin{array}{l}\sigma \\
0 \\
\circ\end{array}$ & $\begin{array}{c}0 \\
0 \\
0\end{array}$ \\
\hline 客 & & $\begin{array}{l}\infty \\
8 \\
8 \\
0 \\
-i\end{array}$ & $\begin{array}{l}50 \\
m 58\end{array}$ & 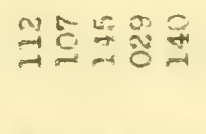 & 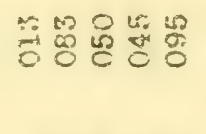 & 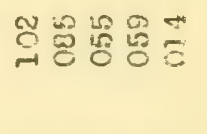 & 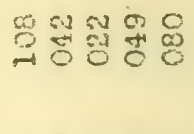 \\
\hline 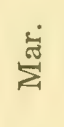 & & $\begin{array}{l}5 \\
8 \\
8 \\
0 \\
-1\end{array}$ & 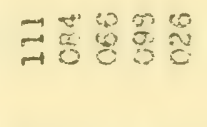 & 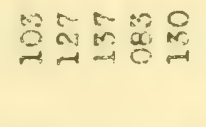 & $\begin{array}{l}60 \\
6808 \\
680\end{array}$ & 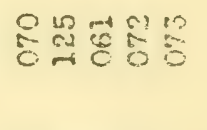 & 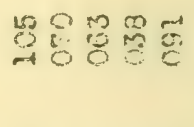 \\
\hline$\frac{\dot{0}}{0}$ & & $\begin{array}{l}7 \\
0 \\
0 \\
0 \\
-1\end{array}$ & 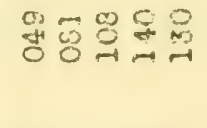 & 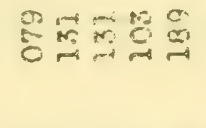 & 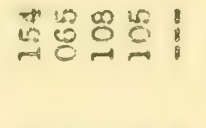 & 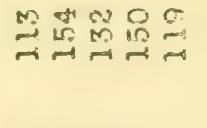 & 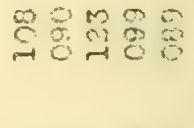 \\
\hline 豞 & & $\begin{array}{l}m \\
\stackrel{m}{0} \\
0 \\
-1\end{array}$ & 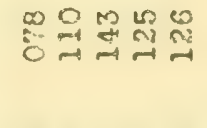 & 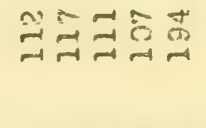 & 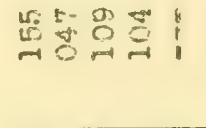 & 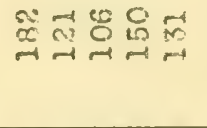 & $\begin{array}{l}400 \\
m b 0\end{array}$ \\
\hline ङ & & $\begin{array}{l}\ddot{n} \\
\ddot{\sim} \\
\sim\end{array}$ & 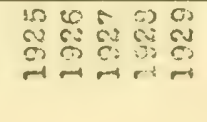 & 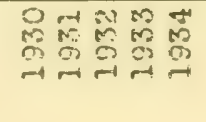 & 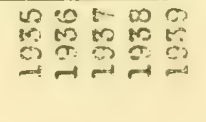 & 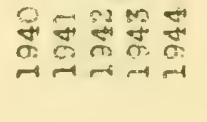 & 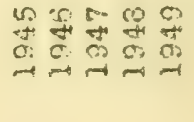 \\
\hline
\end{tabular}




\begin{tabular}{|c|c|c|c|c|c|c|c|c|c|c|}
\hline 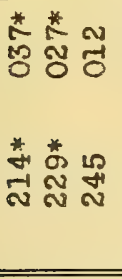 & & 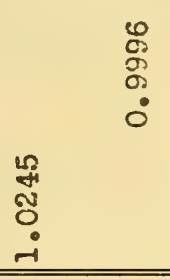 & & 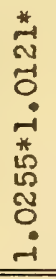 & 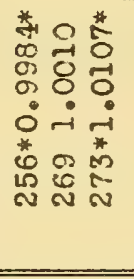 & & 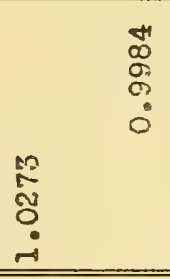 & & 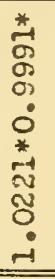 & 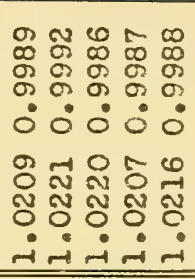 \\
\hline $\begin{array}{l:l} & 0 \\
1 & 1\end{array}$ & $\begin{array}{l}N 0 \\
N \\
-1 \\
0 \\
-1\end{array}$ & & & i & $\begin{array}{l}10 \\
10 \\
0 \\
0 \\
0\end{array}$ & 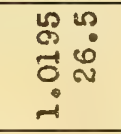 & & & $!$ & 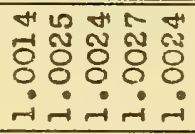 \\
\hline î & $\begin{array}{l}-1 \\
\text { 10 } \\
-1 \\
0 \\
0\end{array}$ & 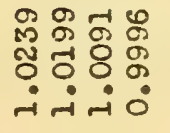 & & $\begin{array}{l}\infty \\
1 \\
0 \\
0 \\
-1\end{array}$ & 式式误 & 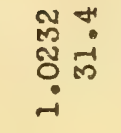 & 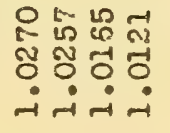 & & मे & 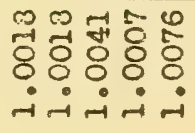 \\
\hline เి & $\begin{array}{l}\qquad 0 \\
0 \\
-1 \\
0 \\
0 \\
-1\end{array}$ & 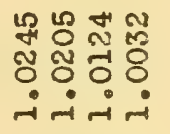 & $\dot{<}$ & $\begin{array}{l}\text { Nิ } \\
\text { ปิ } \\
\text { - }\end{array}$ & 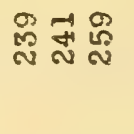 & 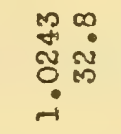 & 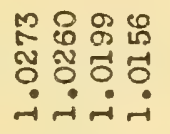 & & $\begin{array}{l}\frac{12}{8} \\
-i\end{array}$ & 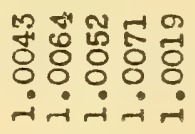 \\
\hline is & $\begin{array}{l}\text { m } \\
\text { 10 } \\
0 \\
0 \\
0\end{array}$ & 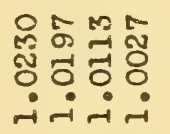 & ச) & 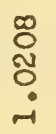 & 웜 & 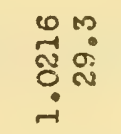 & 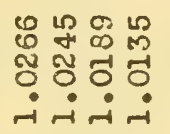 & & $\begin{array}{l}\text { ญै } \\
\stackrel{-}{-1}\end{array}$ & 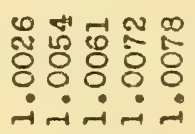 \\
\hline 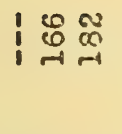 & 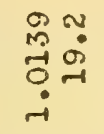 & 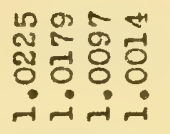 & $\frac{\mathscr{E}}{E}$ & $\begin{array}{l}\text { m } \\
\text { Õ } \\
\text { i- }\end{array}$ & ब 8 \& & 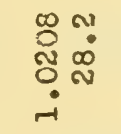 & 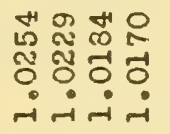 & $\dot{\zeta}$ & $\begin{array}{l}0 \\
8 \\
8 \\
2\end{array}$ & 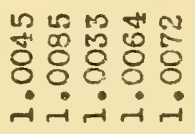 \\
\hline 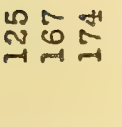 & $\begin{array}{ll}m & \infty \\
-1 & 0 \\
0 & 0 \\
-1 & -1\end{array}$ & 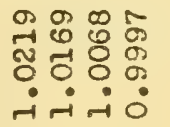 & $\begin{array}{l}\infty \\
\frac{E}{2} \\
\end{array}$ & $\begin{array}{l}\dot{0} \\
\text { N } \\
\text { : } \\
-i\end{array}$ & 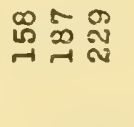 & 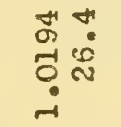 & 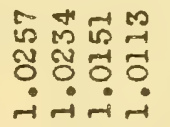 & $\frac{9}{2}$ & $\begin{array}{l}n \\
\text { ? } \\
8 \\
0\end{array}$ & 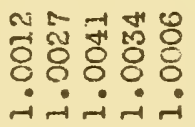 \\
\hline 㘥点 & $\begin{array}{ll}-1 & 0 \\
n & 0 \\
0 & 0 \\
0 & 0 \\
-1\end{array}$ & 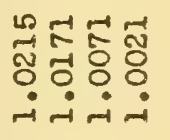 & $\begin{array}{l}\frac{\pi}{a} \\
\frac{\pi}{c} \\
.0\end{array}$ & $\begin{array}{l}n \\
\infty \\
-1 \\
0 \\
-1\end{array}$ & $1 \stackrel{0}{\circ}$ & $\begin{array}{l}N \\
\infty \\
\infty \\
0 \\
0 \\
-i\end{array}$ & 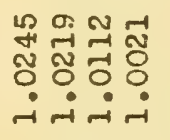 & $\frac{\bar{\omega}}{\sum_{\bar{w}}}$ & $\begin{array}{l}0 \\
-1 \\
8 \\
-1\end{array}$ & 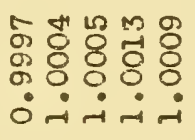 \\
\hline 离苟 & $\begin{array}{l}0 \\
0 \\
0 \\
0 \\
0 \\
-1\end{array}$ & 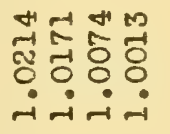 & 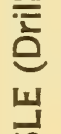 & \begin{tabular}{l}
$\infty$ \\
\multirow{2}{0}{} \\
0 \\
-1
\end{tabular} & iे & $\begin{array}{l}\stackrel{+}{\infty} \underset{-1}{\infty} \\
-1 \\
0 \\
-1\end{array}$ & 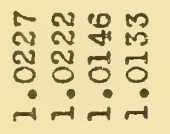 & 山 & $\begin{array}{l}\infty \\
\text { क } \\
\sigma \\
\sigma \\
\dot{0}\end{array}$ & 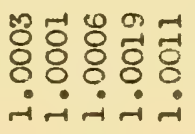 \\
\hline 为品 \& & $\begin{array}{l}0 \\
0 \\
0 \\
0 \\
-1\end{array}$ & 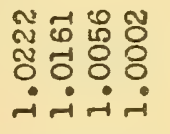 & $\frac{n}{2}$ & $\begin{array}{l}0 \\
\frac{1}{0} \\
-1\end{array}$ & ! & 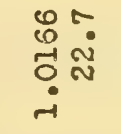 & 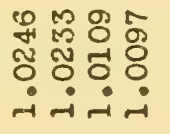 & & $\begin{array}{l}-1 \\
8 \\
8 \\
-1\end{array}$ & 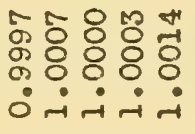 \\
\hline 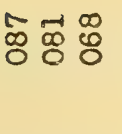 & $\begin{array}{l}0 \\
0 \\
0 \\
0\end{array}$ & 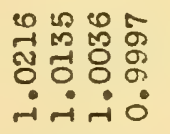 & $\frac{\alpha}{v}$ & i & $\begin{array}{l}5 \\
\infty \\
0 \\
0 \\
0 \\
-1\end{array}$ & 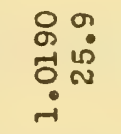 & 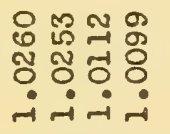 & & $\begin{array}{l}\text { 연 } \\
8 \\
-1\end{array}$ & 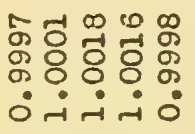 \\
\hline 品出。 & $\begin{array}{l}0 \\
8 \\
8 \\
0 \\
0 \\
\dot{i}\end{array}$ & 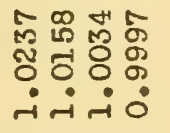 & & i & $\begin{array}{l}10 \\
10 \\
1 \\
0 \\
0 \\
0-1\end{array}$ & 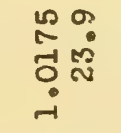 & 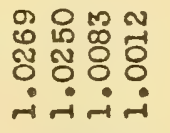 & & $i$ & 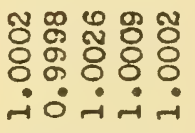 \\
\hline 赵: & $\begin{array}{l}0 \\
\stackrel{0}{-1} \\
0 \\
-1\end{array}$ & 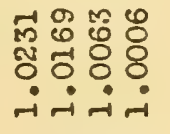 & & ! & 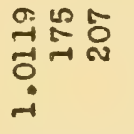 & $\begin{array}{l}5 \\
6 \\
-1 \\
0 \\
0 \\
-1\end{array}$ & 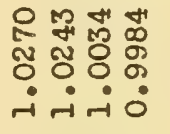 & & i & 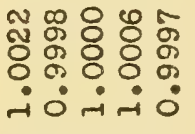 \\
\hline 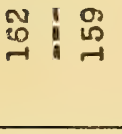 & 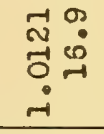 & 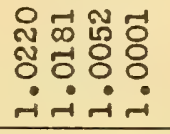 & & i & 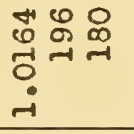 & 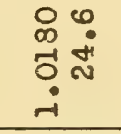 & 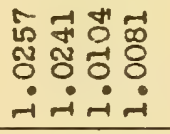 & & i & 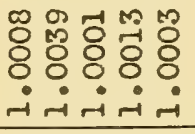 \\
\hline 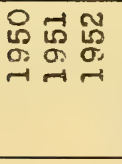 & 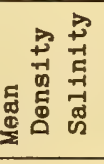 & 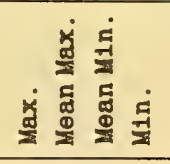 & & $\underset{\sigma}{g}$ & 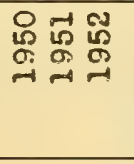 & 常 & 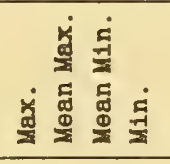 & & क्ष & 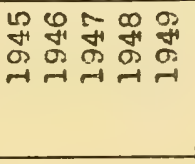 \\
\hline
\end{tabular}




\begin{tabular}{|c|c|c|c|c|c|c|c|c|}
\hline હं & & 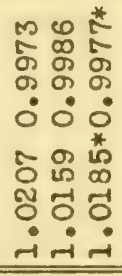 & & $\begin{array}{l}-1 \\
\widetilde{N} \\
\text { O } \\
-1 \\
\end{array}$ & & 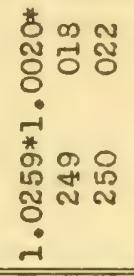 & 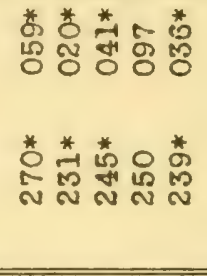 & 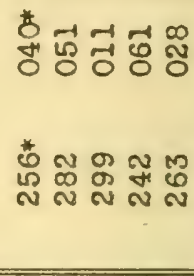 \\
\hline 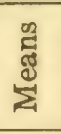 & & $\begin{array}{l}0 \\
0 \\
0\end{array}$ & 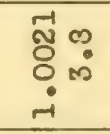 & & & $\begin{array}{l}0 \\
1 \\
1 \\
0 \\
0 \\
0\end{array}$ & !: $: E$ i & ! बेฐ \\
\hline சீं & & 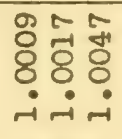 & $\begin{array}{l}0 \\
0 \\
0 \\
0 \\
0 \\
0.1\end{array}$ & 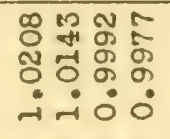 & & 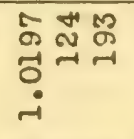 & 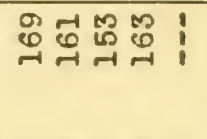 & 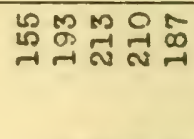 \\
\hline z: & & 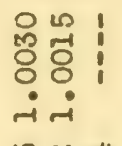 & 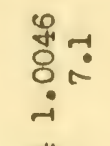 & 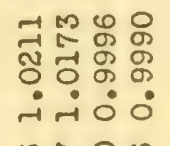 & & 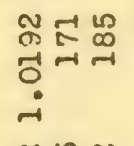 & 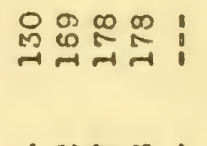 & 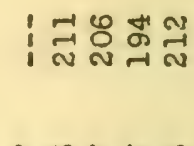 \\
\hline ö & & 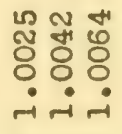 & $\begin{array}{l}+1 \\
0 \\
0\end{array}$ & 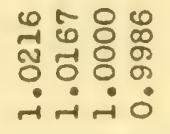 & & 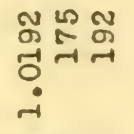 & i & $\overbrace{-1}^{\infty} \underset{\omega}{\omega} 5 \stackrel{-\infty}{\omega}$ \\
\hline 苍 & 总 & $\begin{array}{l}\text { 덩 ญु } \\
8 \\
\circ\end{array}$ & $\begin{array}{l}\text { 요 } \\
0 \\
0 \\
0\end{array}$ & 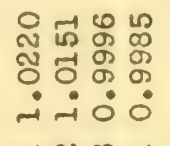 & & 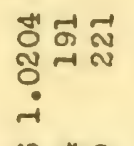 & 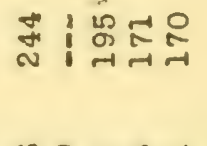 & 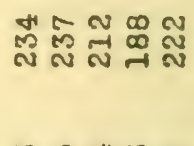 \\
\hline$\stackrel{8}{4}_{4}^{\circ 0}$ & $\dot{3}$ & 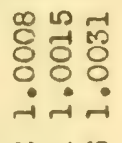 & 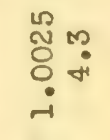 & 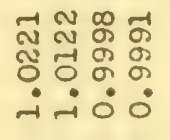 & zu & 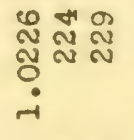 & ڤొ & 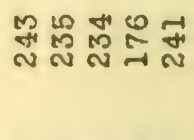 \\
\hline$\frac{2}{3}$ & $\frac{0}{\frac{1}{5}}$ & 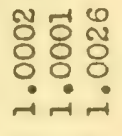 & ㄷㅇㅇㅛ & 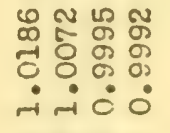 & $\begin{array}{l}\frac{5}{u} \\
\frac{u}{2}\end{array}$ & 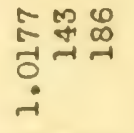 & 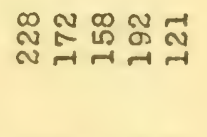 & 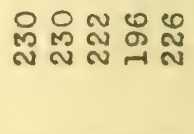 \\
\hline$\underset{\Xi}{\Xi}$ & 岁 & 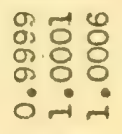 & $\begin{array}{l}0 \\
8 \\
8 \\
0 \\
-1\end{array}$ & 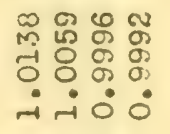 & (1) & 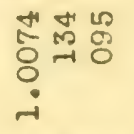 & बू̃ & 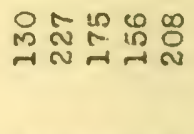 \\
\hline$\sum_{i=1}^{\infty}$ & $\vec{\omega}$ & 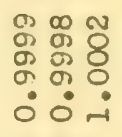 & $\begin{array}{l}v \\
\tilde{c} \\
0 \\
0 \\
\dot{0}\end{array}$ & 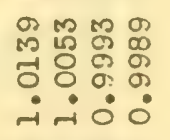 & & 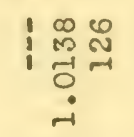 & 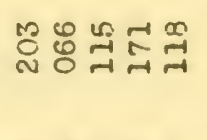 & 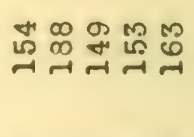 \\
\hline 安 & & 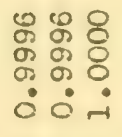 & $\begin{array}{l}50 \\
80 \\
0\end{array}$ & 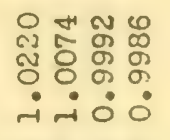 & & 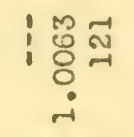 & 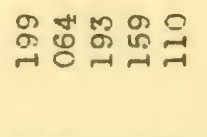 & 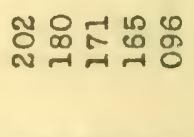 \\
\hline$\sum^{\dot{\sigma}}$ & & 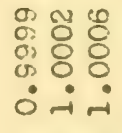 & $\begin{array}{l}8 \\
8 \\
0 \\
0\end{array}$ & 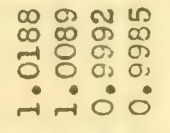 & & 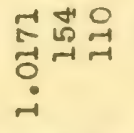 & 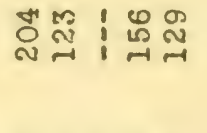 & 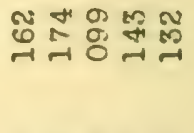 \\
\hline$\frac{\dot{0}}{\dot{0}}$ & & 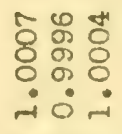 & $\begin{array}{l}4 \\
8 \\
0 \\
0\end{array}$ & 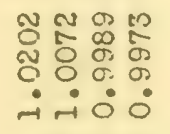 & & 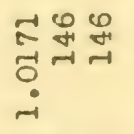 & 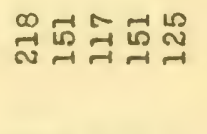 & 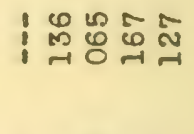 \\
\hline 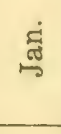 & & $\begin{array}{l}m \\
0 \\
0 \\
0 \\
0 \\
0\end{array}$ & $\begin{array}{l}+10 \\
80 \\
0\end{array}$ & 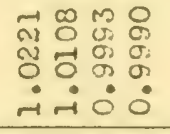 & & 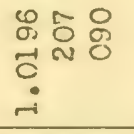 & 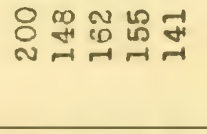 & 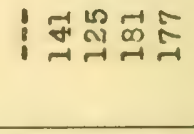 \\
\hline ฮึ & & 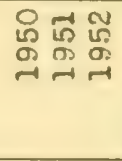 & 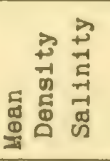 & 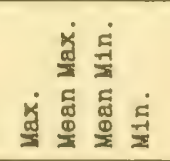 & & 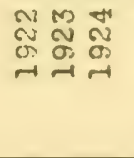 & 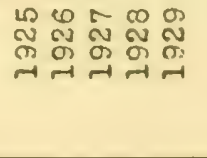 & 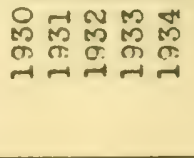 \\
\hline
\end{tabular}




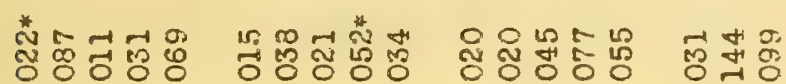

*

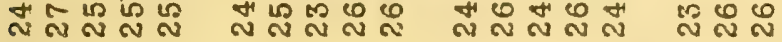

एक

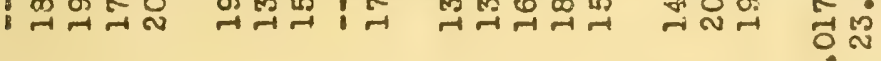

\begin{tabular}{|c|c|c|c|c|}
\hline చై & 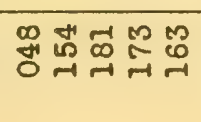 & 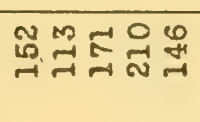 & స్రం & 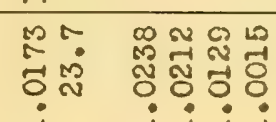 \\
\hline
\end{tabular}

H How

क $00-1$ t

न

-

ம

-

ธี त

i

ตับนี⿻

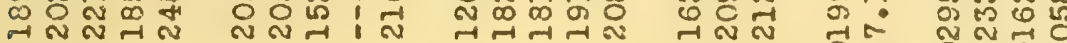

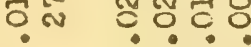

i

0 क स

约药

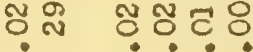

i

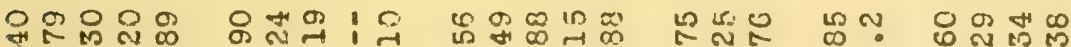

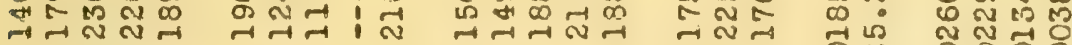

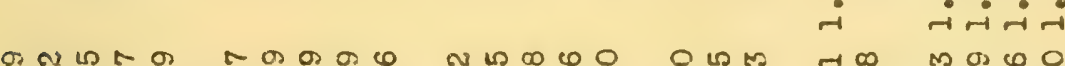

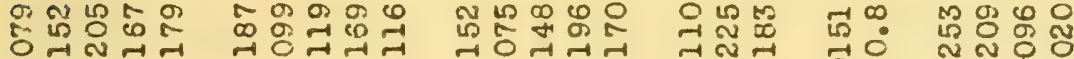

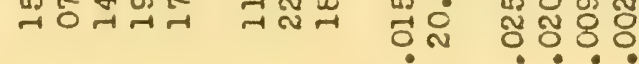

-i

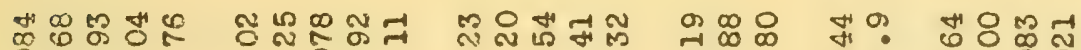

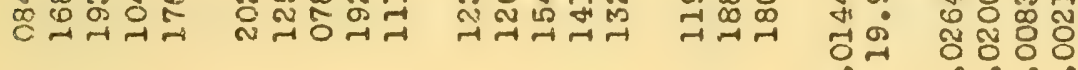

Hom

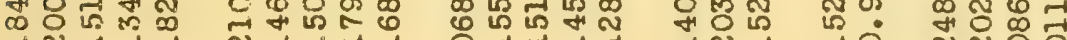

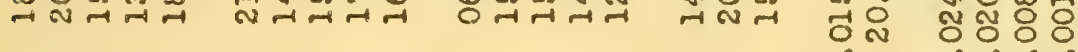

1

เి

i

m

Hह

है

గదำ

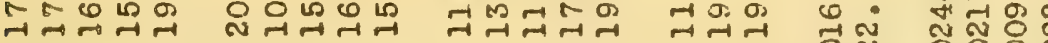

(5)

-1

- 0000

以

-1 -1

ㄴ.

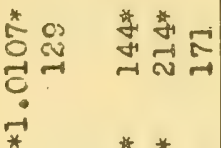

菅

mo m $\mathrm{m}$ 


\begin{tabular}{|c|c|c|c|c|c|c|c|c|c|}
\hline 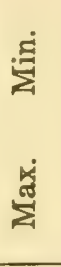 & & & $\begin{array}{l}5 \\
5 \\
8 \\
-1\end{array}$ & & 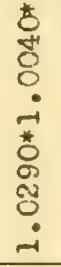 & 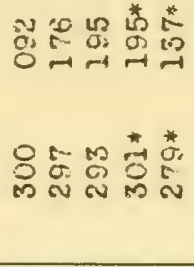 & 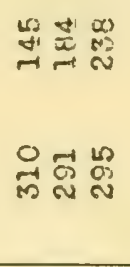 & & 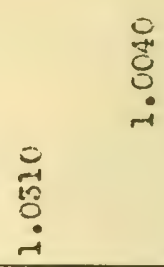 \\
\hline 荧 & & 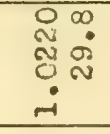 & & & i & 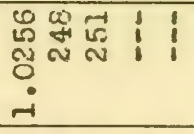 & బั & $\begin{array}{l}4 \\
\widetilde{0} \\
\dddot{0}\end{array}$ & \\
\hline ஜ் & & $\begin{array}{l}\infty \\
\infty\end{array}$ & 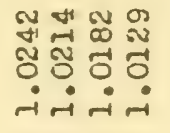 & & $\begin{array}{l}\text { N్ } \\
\text { Õ } \\
0 \\
-1\end{array}$ & 芯心岕! 岕 & N & 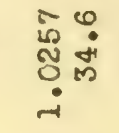 & 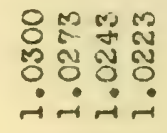 \\
\hline 嵒 & & 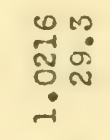 & 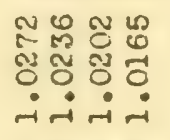 & & 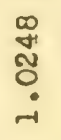 & డ్రం & 点芯点 & $\begin{array}{l}-1 \\
0 \\
0 \\
0 \\
0\end{array}$ & 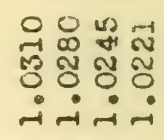 \\
\hline$\dot{0}$ & & 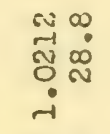 & 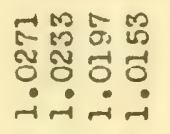 & & $\begin{array}{l}10 \\
\text { N } \\
0 \\
-1\end{array}$ & 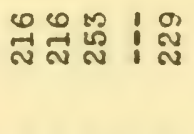 & 蛋心 & $\begin{array}{l}50 \\
m: \\
\tilde{~} \\
0 \\
i\end{array}$ & 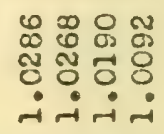 \\
\hline 苋 & 导 & 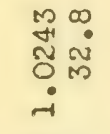 & 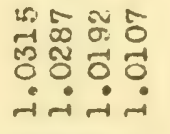 & & 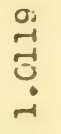 & 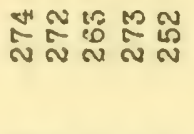 & 윤 & 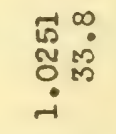 & 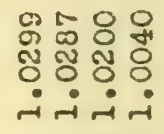 \\
\hline$\stackrel{8}{3}_{4}^{\circ}$ & $\begin{array}{l}\text { ¿ } \\
\dot{y} \\
\dot{u}\end{array}$ & 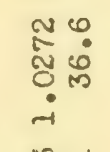 & 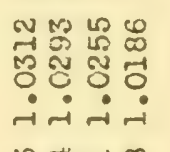 & 닙 & 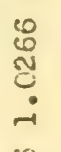 & 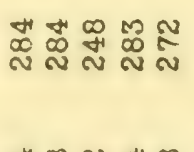 & 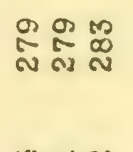 & 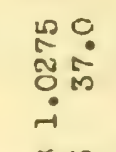 & 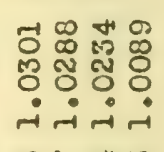 \\
\hline$\frac{2}{3}$ & 㟶 & 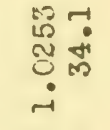 & 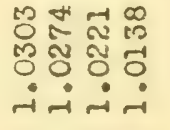 & $\frac{\sqrt{a}}{n}$ & 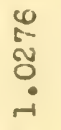 & 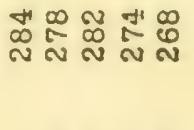 & 䟢柋 & कृ & 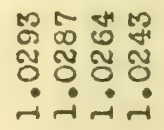 \\
\hline$\stackrel{\Xi}{\leftrightarrows}$ & $\begin{array}{l}\text { x } \\
\frac{1}{0} \\
0 \\
x\end{array}$ & 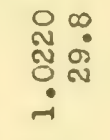 & 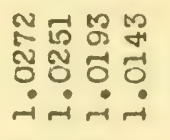 & 옹 & 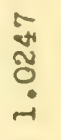 & 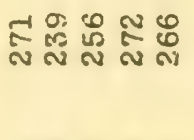 & 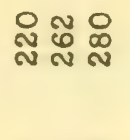 & సี & 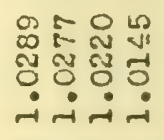 \\
\hline 㞼 & & $\begin{array}{l}\hat{0} \\
\mathbb{N} \\
0 \\
\dot{0}\end{array}$ & 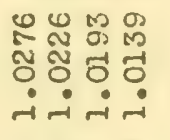 & & $\begin{array}{l}\mathscr{O} \\
\stackrel{\sim}{0} \\
0 \\
-\end{array}$ & 㖼志筧: & 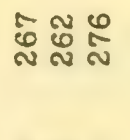 & 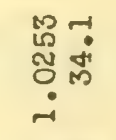 & 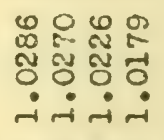 \\
\hline 䢓 & & 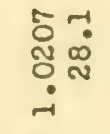 & 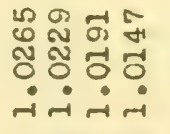 & & 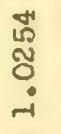 & 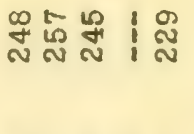 & 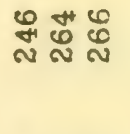 & 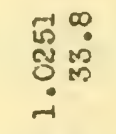 & 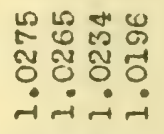 \\
\hline 宓 & & 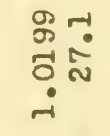 & 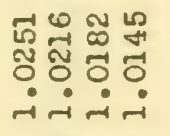 & & i & 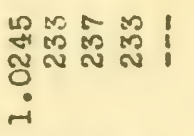 & 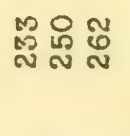 & 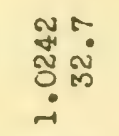 & 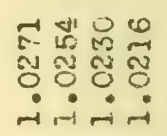 \\
\hline$\dot{0}$ & & 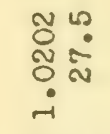 & 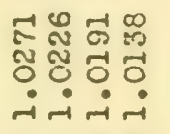 & & i & 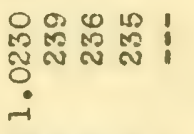 & 吉罟心 & \begin{tabular}{l}
$m \infty$ \\
\multirow{4}{*}{$心$} \\
$\tilde{i}$ \\
$\dot{i}$
\end{tabular} & 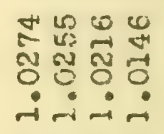 \\
\hline 楽 & & 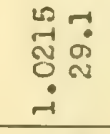 & 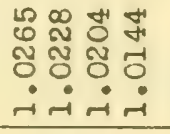 & & i & 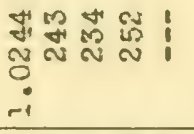 & 誌忥志 & 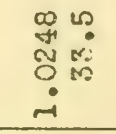 & 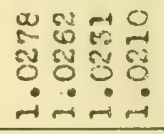 \\
\hline 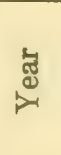 & & 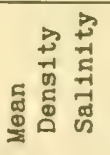 & 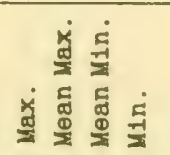 & & $\underset{\sim}{\stackrel{H}{*}}$ & 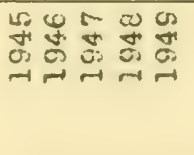 & 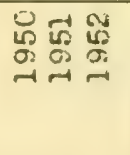 & 索孛 & 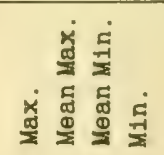 \\
\hline
\end{tabular}




\begin{tabular}{|c|c|c|c|c|c|c|c|c|c|}
\hline & 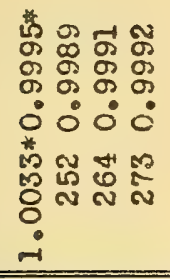 & 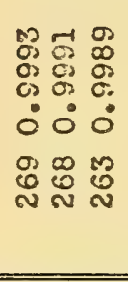 & & 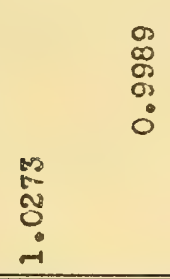 & & 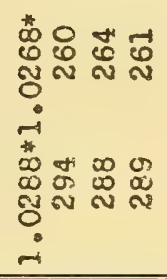 & 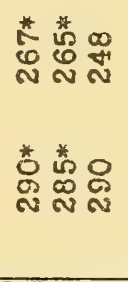 & & 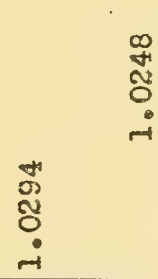 \\
\hline & 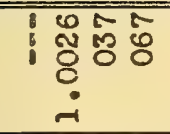 & 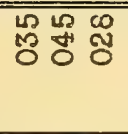 & $\begin{array}{l}0 \\
8 \\
8 \\
8 \\
0\end{array}$ & & & 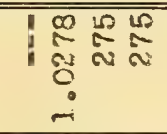 & !I: & ํㅜㅇ & \\
\hline \multirow{13}{*}{ 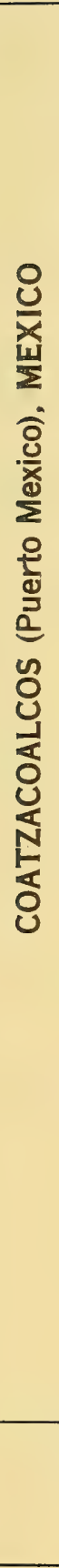 } & 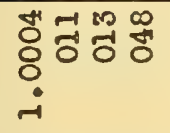 & ๙ొరి & ${ }_{i}^{\infty}$ & 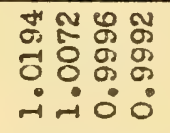 & \multirow{12}{*}{ 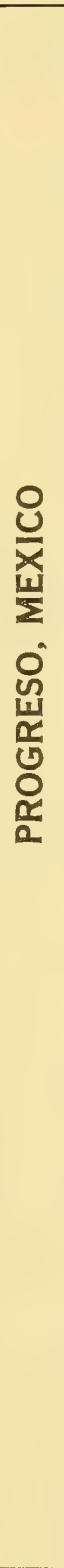 } & 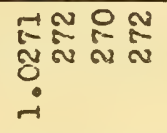 & 施是 & 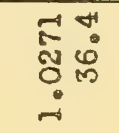 & 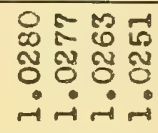 \\
\hline & 둉요용 & 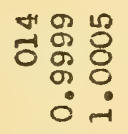 & $\frac{8}{8}$ & 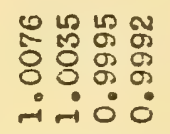 & & 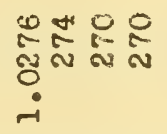 & M & 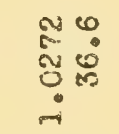 & 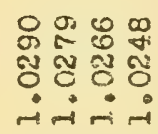 \\
\hline & 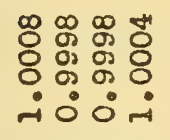 & 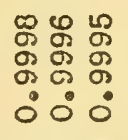 & $\begin{array}{l}8 . \\
8 \\
0 \\
-1\end{array}$ & 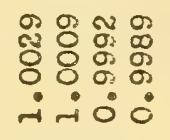 & & 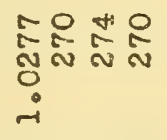 & 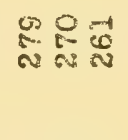 & 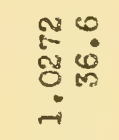 & 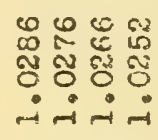 \\
\hline & 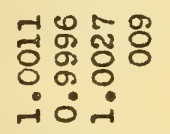 & 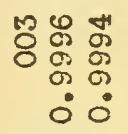 & $\begin{array}{l}0 \\
8 \\
8 \\
0\end{array}$ & 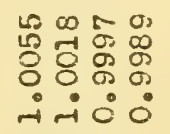 & & 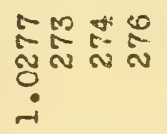 & 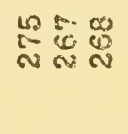 & $\begin{array}{l}20 \\
0 \\
0 \\
0 \\
0\end{array}$ & 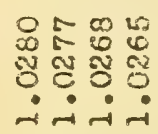 \\
\hline & 둉 & 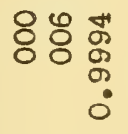 & $\begin{array}{l}\infty \\
-1 \\
8 \\
0 \\
-1\end{array}$ & 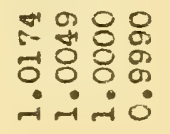 & & 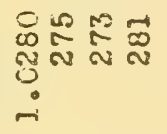 & 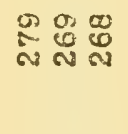 & $\begin{array}{l}400 \\
0 \\
0 \\
0 \\
0 \\
0\end{array}$ & 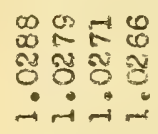 \\
\hline & | & 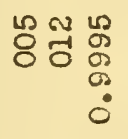 & $\begin{array}{l}-1 \\
0 \\
0\end{array}$ & 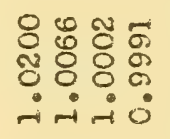 & & 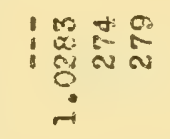 & 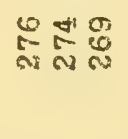 & $\tilde{\tilde{U}}_{i}^{\infty}$ & 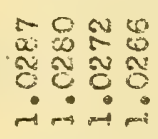 \\
\hline & 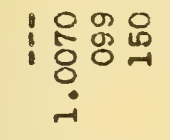 & 出号峁 & $\begin{array}{l}\text { मे } 0 \\
0 \\
0 \\
0 \\
0 \\
-1\end{array}$ & 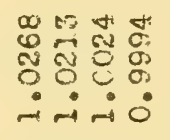 & & 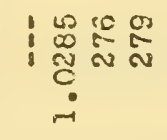 & 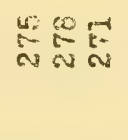 & $\frac{\pi}{0}=$ & 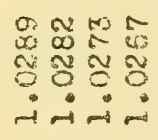 \\
\hline & 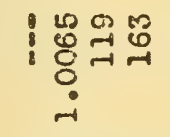 & 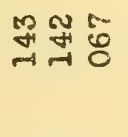 & $\begin{array}{l}0 \\
-1 \\
-1 \\
0 \\
-i\end{array}$ & 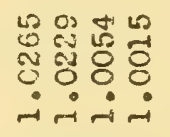 & & 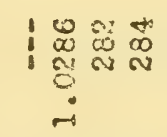 & 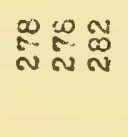 & 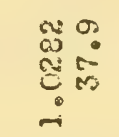 & 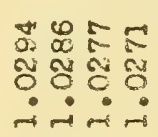 \\
\hline & 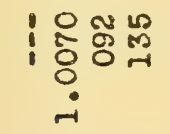 & 옹휴웜 & $\begin{array}{l}-\pi \\
8 \\
8 \\
8\end{array}$ & 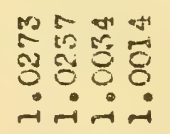 & & 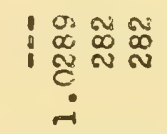 & în & $\begin{array}{l}m \\
2 \\
0 \\
0 \\
0 \\
\dot{0}\end{array}$ & 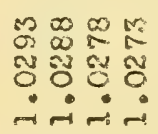 \\
\hline & 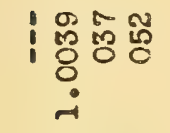 & $\underset{\forall}{\infty} \& \stackrel{D}{\circ}$ & 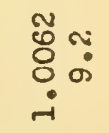 & 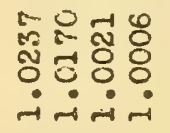 & & 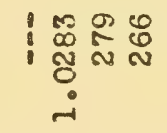 & 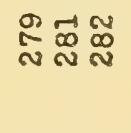 & 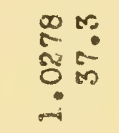 & 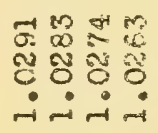 \\
\hline & | & 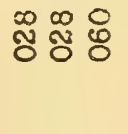 & मี & 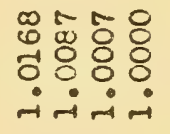 & & 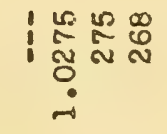 & ล: & $\begin{array}{l}50 \\
5:-5 \\
0\end{array}$ & 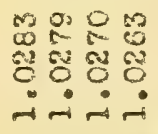 \\
\hline & $\begin{array}{l}98 \\
18 \\
8 \\
8 \\
0 \\
-1\end{array}$ & 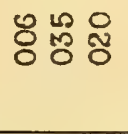 & ${ }_{0}^{0}$ & 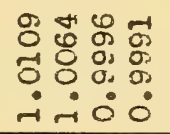 & & 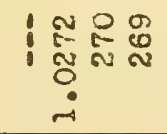 & 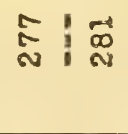 & 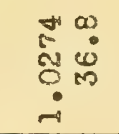 & 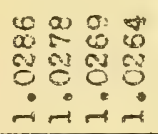 \\
\hline & 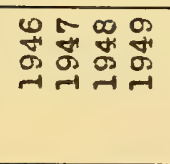 & 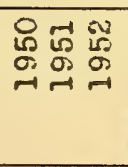 & 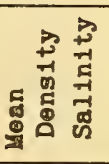 & 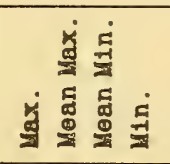 & & 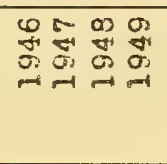 & 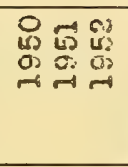 & 总 & 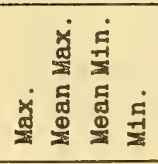 \\
\hline
\end{tabular}




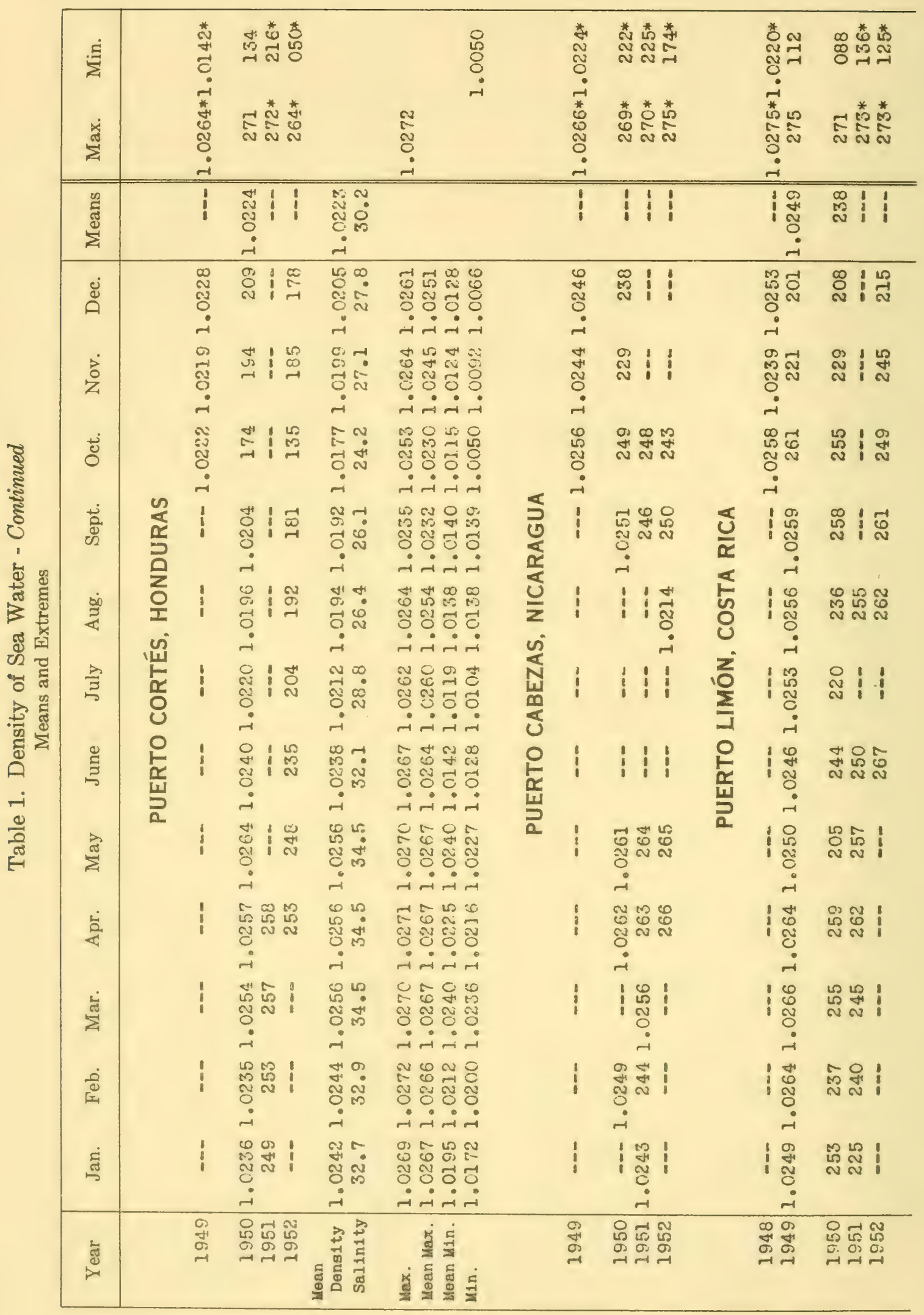




\begin{tabular}{|c|c|c|c|c|c|c|c|c|c|}
\hline & $\begin{array}{l}\infty \\
0 \\
0 \\
0 \\
i\end{array}$ & & 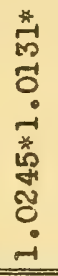 & 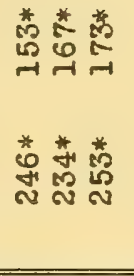 & & $\begin{array}{l}\text { : } \\
\text { Oี } \\
0 \\
-1 \\
\end{array}$ & & 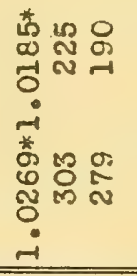 & 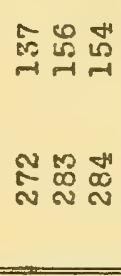 \\
\hline 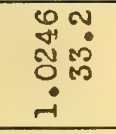 & & & $i$ & $1 i 1$ & 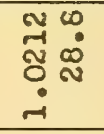 & & & 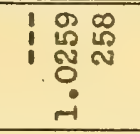 & 岇㫄占 \\
\hline 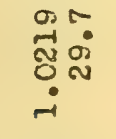 & 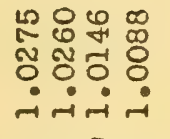 & & $\begin{array}{l}5 \\
0 \\
0 \\
0 \\
-1\end{array}$ & 1 & $\begin{array}{l}0: \\
0 \\
0 \\
0 \\
\dot{1}\end{array}$ & 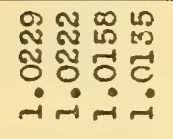 & & 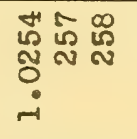 & 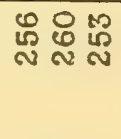 \\
\hline 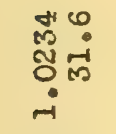 & 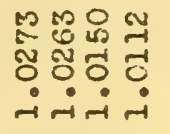 & & $\begin{array}{l}0 \\
0 \\
5 \\
0 \\
-1\end{array}$ & Sి & 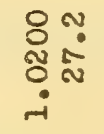 & 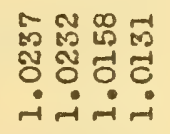 & & 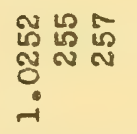 & 蛋怘 \\
\hline 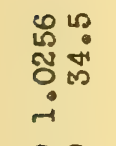 & 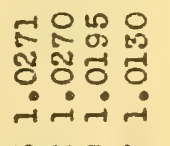 & & $\begin{array}{l}\mathbb{m} \\
\text { N } \\
0 \\
0 \\
-1\end{array}$ & 心 & 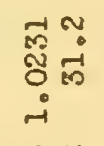 & 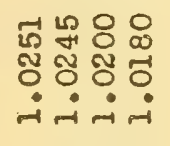 & & 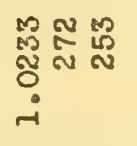 & 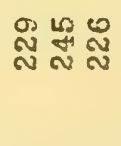 \\
\hline 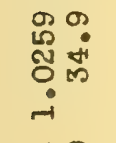 & 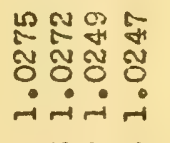 & $\frac{4}{2}$ & 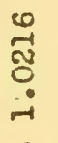 & il & $\begin{array}{l}\text { N } \\
\text { U. } \\
0 \\
0 \\
-1\end{array}$ & 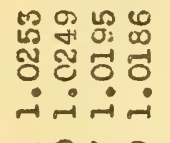 & & 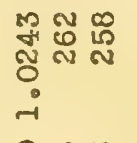 & 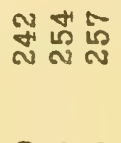 \\
\hline 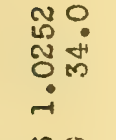 & 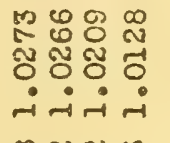 & $\frac{1}{3}$ & $\begin{array}{l}\mathscr{8} \\
\mathscr{円} \\
-i\end{array}$ & 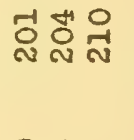 & 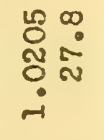 & 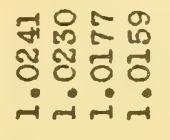 & $\frac{\pi}{0}$ & 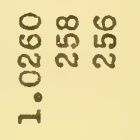 & 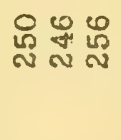 \\
\hline $\begin{array}{ll}0 & 0: \\
10 & 0 \\
0 & \text { ल }\end{array}$ & 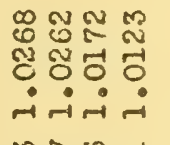 & $\frac{1}{0}$ & ! & 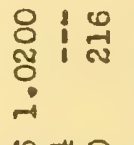 & 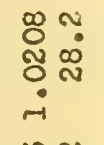 & 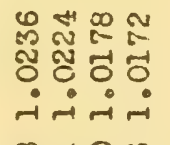 & $\sum_{1}^{\frac{1}{2}}$ & 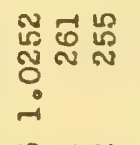 & 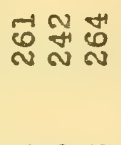 \\
\hline $\begin{array}{l}\text { No } \\
\text { W } \\
\text { U } \\
\text { i } \\
\text { i }\end{array}$ & 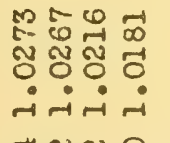 & $\frac{5}{\frac{5}{u}}$ & i & 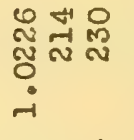 & 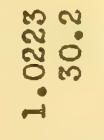 & 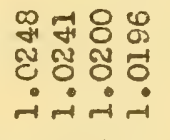 & & 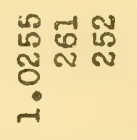 & 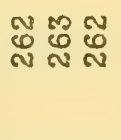 \\
\hline $\begin{array}{l}50 \\
\text { No } \\
\tilde{O} \text { N } \\
i\end{array}$ & 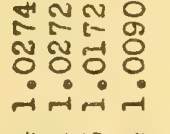 & & i & 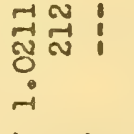 & $\begin{array}{l}N \\
-1 \\
\tilde{N} \\
0 \\
-i \\
-1\end{array}$ & 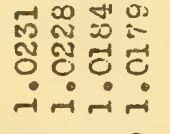 & & 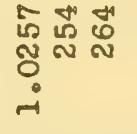 & 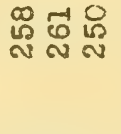 \\
\hline 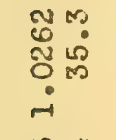 & 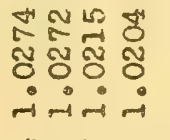 & & $!$ & 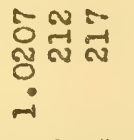 & 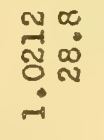 & 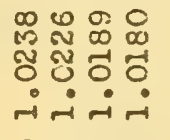 & & 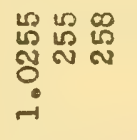 & 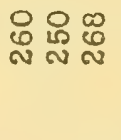 \\
\hline 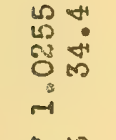 & 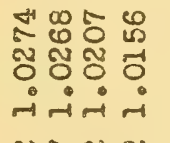 & & i & 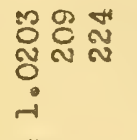 & 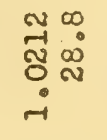 & 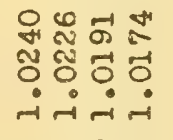 & & 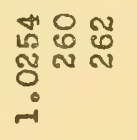 & 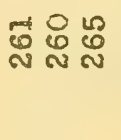 \\
\hline $\begin{array}{l}-5 \\
\text { Hi } \\
0 \\
0 \\
0\end{array}$ & 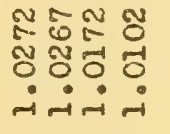 & & i & 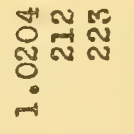 & $\begin{array}{ll}\infty & 0 \\
-1 & 0 \\
\tilde{0} & 0 \\
-1 & 0\end{array}$ & 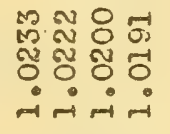 & & $\begin{array}{l}\infty \\
100 \\
0 \\
0 \\
0 \\
0 \\
-1\end{array}$ & 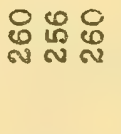 \\
\hline $\begin{array}{l}\tilde{J} \\
\tilde{O} \\
\tilde{O}\end{array}$ & 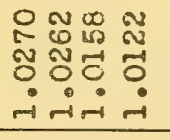 & & i & 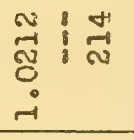 & $\begin{array}{l}m \\
-1 \\
\sim \\
0 \\
0 \\
-1\end{array}$ & 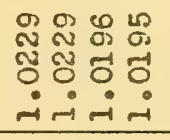 & & $\begin{array}{lll}1 & 0 & 0 \\
1 & 15 \\
1 & 0 \\
0 & 0 \\
0 \\
.1\end{array}$ & 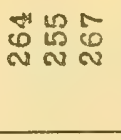 \\
\hline 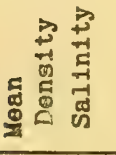 & 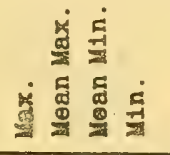 & & $\underset{\varpi}{\mathscr{g}}$ & 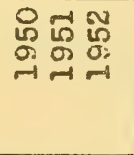 & 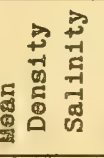 & 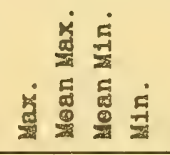 & & 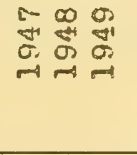 & 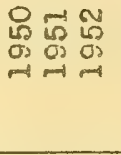 \\
\hline
\end{tabular}




\begin{tabular}{|c|c|c|c|c|c|c|c|c|c|c|c|}
\hline 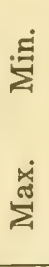 & & & $\begin{array}{l}n \\
0 \\
8 \\
0 \\
-\end{array}$ & & 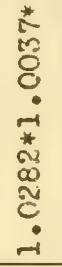 & 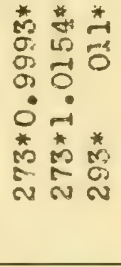 & & 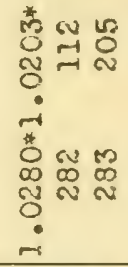 & 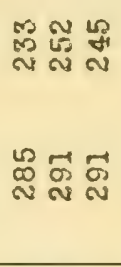 & & $\begin{array}{l}\vec{\sigma} \\
\tilde{0} \\
\dot{-1}\end{array}$ \\
\hline 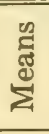 & & 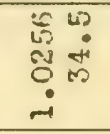 & & & $!$ & $\begin{array}{l:l} & 1 \\
1 & 1\end{array}$ & & 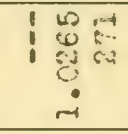 & 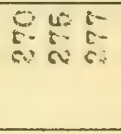 & $\begin{array}{ll}\sigma & 0 \\
2 & 0 \\
\tilde{c} & 0 \\
-1 & m \\
-1\end{array}$ & \\
\hline 巳્ઁ் & & 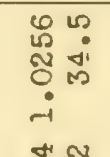 & 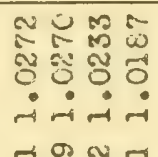 & & $\begin{array}{l}n \\
\text { N } \\
\tilde{0} \\
-1 \\
0\end{array}$ & 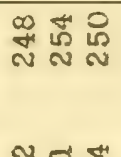 & & 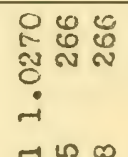 & 过芯 & 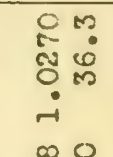 & 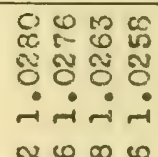 \\
\hline z & & 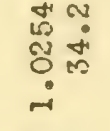 & 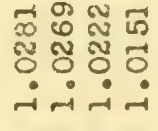 & & 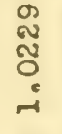 & 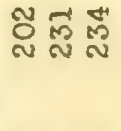 & & 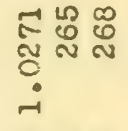 & 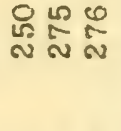 & $\begin{array}{ll}\infty & 0 \\
0 & 0 \\
N & 0 \\
0 & m \\
-1 & 0\end{array}$ & 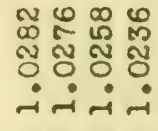 \\
\hline ثึ & & 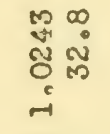 & 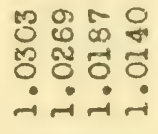 & & ! & 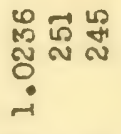 & & 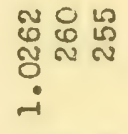 & 虫志悹 & 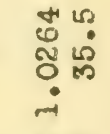 & 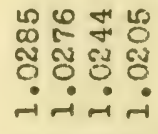 \\
\hline 荵 & ְ. & 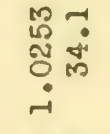 & 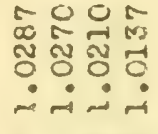 & & i & 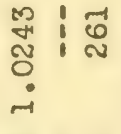 & 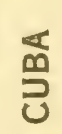 & 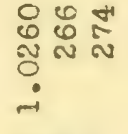 & 选胥 & 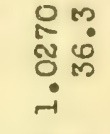 & 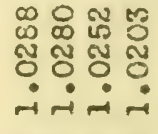 \\
\hline$\stackrel{800}{3}$ & 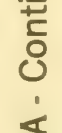 & 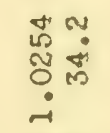 & 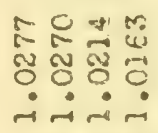 & $\frac{\pi}{0}$ & $!$ & 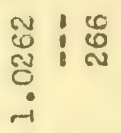 & ¿ั & 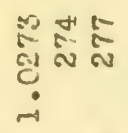 & ลิ & $\underset{\sim}{0} \stackrel{0}{0}$ & 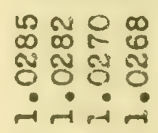 \\
\hline$\frac{2}{3}$ & 号 & 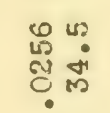 & 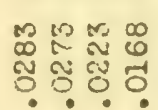 & $\frac{\pi}{\alpha}$ & i & 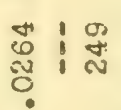 & $\sum_{z}^{0}$ & 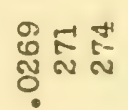 & 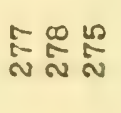 & \begin{tabular}{l}
\multirow{2}{*}{$\infty$} \\
$\tilde{0}$
\end{tabular} & 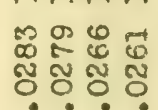 \\
\hline & $\sum$ & 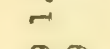 & $-1-1-1$ & v & & & $E$ & & & -1 & $\rightarrow-\dot{-1}$ \\
\hline$\stackrel{\mathscr{\Xi}}{\Xi}$ & 这 & 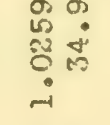 & 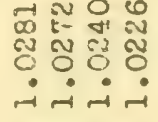 & & $\dot{i}$ & \begin{tabular}{l|l}
0 & 1 \\
0 & 1 \\
0 & 1 \\
- &
\end{tabular} & 蛋 & 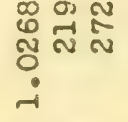 & $\underset{N}{N} \stackrel{-1}{N}$ & 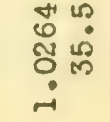 & 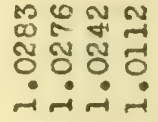 \\
\hline$\sum_{\Sigma}^{\infty}$ & & $\begin{array}{l}\text { to } \\
\text { in } \\
\text { O } \\
-1\end{array}$ & 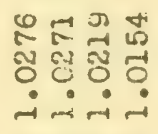 & & $i$ & : i : & & 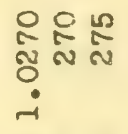 & 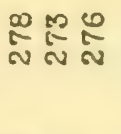 & \begin{tabular}{l}
$+\infty$ \\
\multirow{2}{*}{$\infty$} \\
0 \\
0
\end{tabular} & 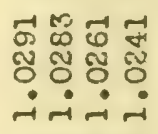 \\
\hline 安 & & $\begin{array}{l}\infty \\
\text { w } \\
\tilde{0} \\
0\end{array}$ & 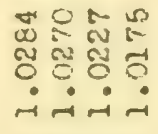 & & 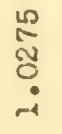 & $i:$ & & $\begin{array}{l}1: 5 \\
0 \\
0 \\
0\end{array}$ & 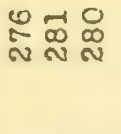 & 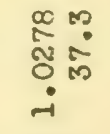 & 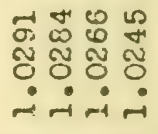 \\
\hline$\sum_{\Sigma}^{\infty}$ & & $\begin{array}{ll}8 & 0 \\
0 & 0 \\
0 & \text { in } \\
0 & \text { in } \\
-1\end{array}$ & 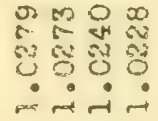 & & i & 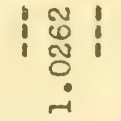 & & 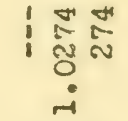 & 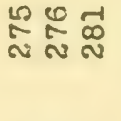 & \begin{tabular}{l}
0 \\
\multirow{2}{*}{} \\
0 \\
0 \\
-1
\end{tabular} & 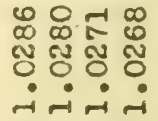 \\
\hline 递 & & 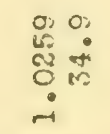 & 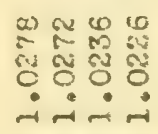 & & 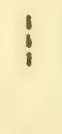 & 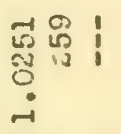 & & 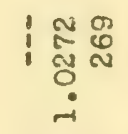 & 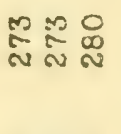 & $\begin{array}{l}m \\
2 \\
0 \\
0 \\
0\end{array}$ & 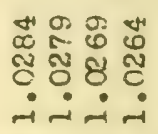 \\
\hline డేี & & 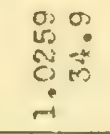 & 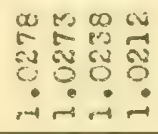 & & i & 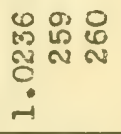 & & 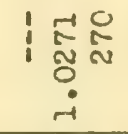 & 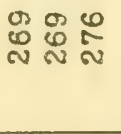 & 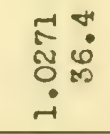 & 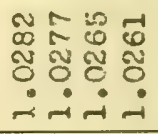 \\
\hline ङ & & 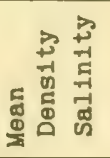 & 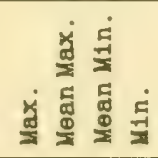 & & $\begin{array}{l}0 . \\
0 \\
0 \\
-4\end{array}$ & 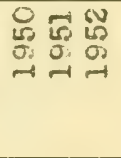 & & 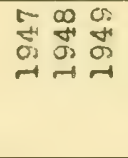 & 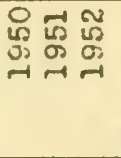 & 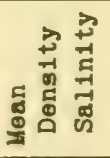 & 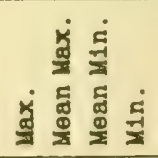 \\
\hline
\end{tabular}




\begin{tabular}{|c|c|c|c|c|c|c|c|c|c|c|c|c|}
\hline & 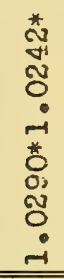 & 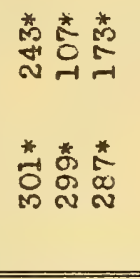 & & $\begin{array}{l}5 \\
0 \\
0 \\
0 \\
-1\end{array}$ & & 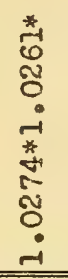 & 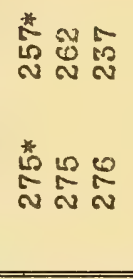 & & $\begin{array}{l}5 \\
0 \\
0 \\
0\end{array}$ & & $\begin{array}{l}5 \\
0 \\
8 \\
8 \\
0 \\
-1 \\
0 \\
0 \\
0 \\
0 \\
0 \\
-1 \\
\end{array}$ & 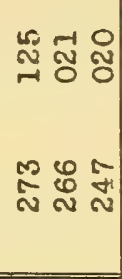 \\
\hline & i & $\begin{array}{lll}1 & 1 & 1\end{array}$ & \begin{tabular}{l}
$\infty$ \\
\multirow{2}{*}{} \\
0 \\
0
\end{tabular} & & & ! & $\begin{array}{l}100 \\
100 \\
0 \\
0 \\
0 \\
0 \\
-1\end{array}$ & $\begin{array}{ll}0 & N \\
0 & 0 \\
N & 0 \\
0 & 0 \\
-i\end{array}$ & & & $i$ & 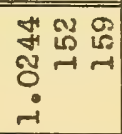 \\
\hline \multirow{13}{*}{ 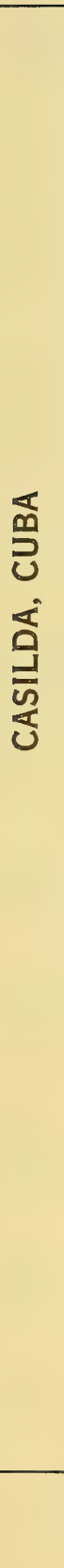 } & 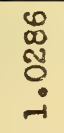 & $\underset{5:}{N}$ & $\begin{array}{l}\overrightarrow{0} \\
0 \\
0 \\
0\end{array}$ & 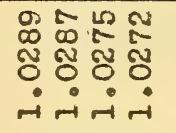 & & 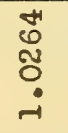 & 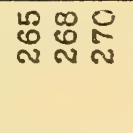 & 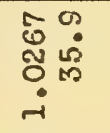 & 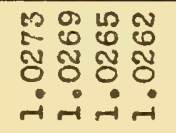 & & 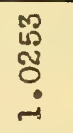 & 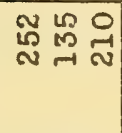 \\
\hline & 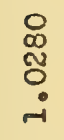 & $\underset{N}{\infty}: \stackrel{\infty}{\infty}$ & 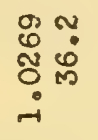 & 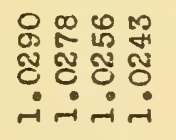 & & $\begin{array}{l}\text { ஸे } \\
\text { Oै } \\
\dot{-1}\end{array}$ & 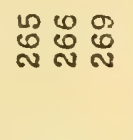 & 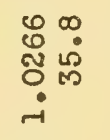 & 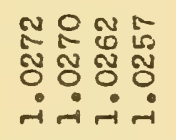 & & 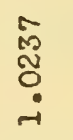 & 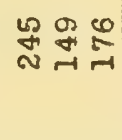 \\
\hline & \begin{tabular}{l}
$\infty$ \\
0 \\
\multirow{O}{0}{} \\
-1
\end{tabular} & : & 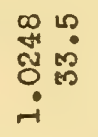 & 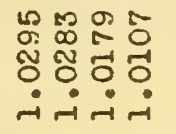 & & 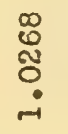 & 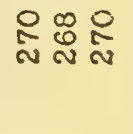 & 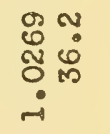 & 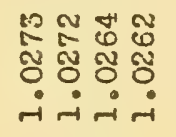 & $\frac{u}{\frac{u}{n}}$ & $\underset{-1}{2}$ & 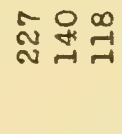 \\
\hline & $\begin{array}{l}\text { on } \\
\text { an } \\
0 \\
-1\end{array}$ & 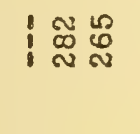 & $\begin{array}{l}\mathscr{O} \\
0 \\
\tilde{O} \\
0 \\
0 \\
i\end{array}$ & 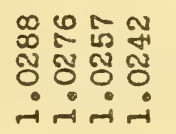 & $\frac{E}{E}$ & $\begin{array}{l}0 \\
0 \\
0 \\
0 \\
\dot{-1}\end{array}$ & 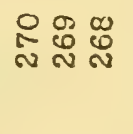 & 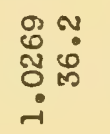 & 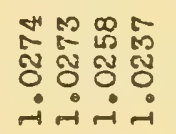 & $\begin{array}{l}\frac{0}{u} \\
\underline{\alpha} \\
z\end{array}$ & $\begin{array}{l}\mathscr{D} \\
0 \\
0 \\
0\end{array}$ & $\stackrel{N}{N} \underset{-1}{\mathbb{N}}$ \\
\hline & 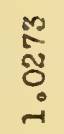 & 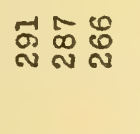 & $\begin{array}{l}9 \\
0 \\
0 \\
0 \\
0\end{array}$ & 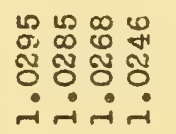 & $\begin{array}{l}I \\
u \\
U \\
\underline{Z}\end{array}$ & i & 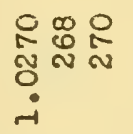 & 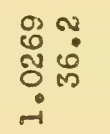 & 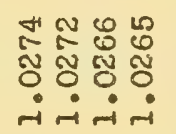 & 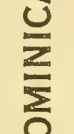 & 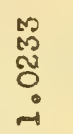 & 怘罢 \\
\hline & 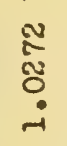 & 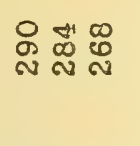 & $\begin{array}{l}\infty \\
0 \\
0 \\
0 \\
0 \\
0\end{array}$ & 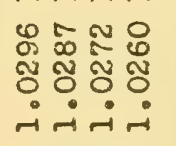 & $\frac{\bar{a}}{\frac{0}{2}}$ & \begin{tabular}{l}
0 \\
\multirow{2}{0}{} \\
0 \\
-1
\end{tabular} & 돈 & $\begin{array}{l}E \\
\text { ¿ } \\
0\end{array}$ & 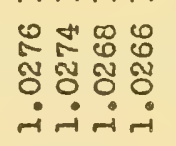 & $\begin{array}{l}0 \\
0 \\
0 \\
3\end{array}$ & 湈 & 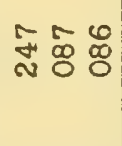 \\
\hline & i & 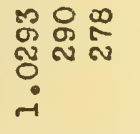 & $\begin{array}{ll}5 & 5 \\
\infty & 0 \\
0 & \infty \\
0 & \\
-1\end{array}$ & 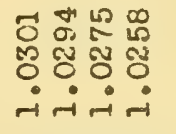 & $\frac{a}{0}$ & 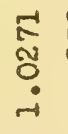 & 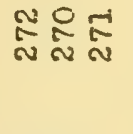 & 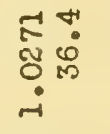 & 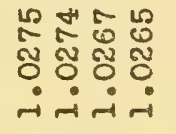 & $\frac{5}{5}$ & 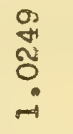 & జ̛ \\
\hline & $\begin{array}{l}n \\
\infty \\
0 \\
0 \\
-1\end{array}$ & $\begin{array}{l}\vec{\sigma} \underset{\infty}{\infty} \vec{\infty} \\
\sim \\
\sim\end{array}$ & $\begin{array}{l}\omega \\
\infty \\
\infty \\
心 \\
0 \\
0 \\
\end{array}$ & 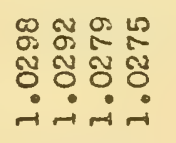 & & $\begin{array}{l}0 \\
\text { Nิ } \\
0 \\
\text { - }\end{array}$ & 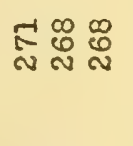 & 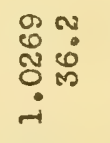 & 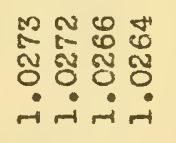 & $\begin{array}{l}\frac{9}{0} \\
2 \\
0\end{array}$ & \begin{tabular}{l}
0 \\
\multirow{2}{*}{} \\
O \\
-1
\end{tabular} & 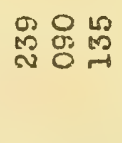 \\
\hline & 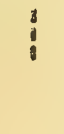 & $\begin{array}{l}\infty \\
\infty \\
\infty \\
N \\
0 \\
0 \\
-i\end{array}$ & $\begin{array}{l}0 \\
\infty \\
\infty \\
心 \\
0 \\
0 \\
\dot{-1}\end{array}$ & 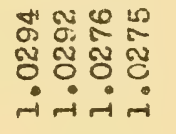 & & i & 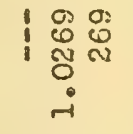 & $\begin{array}{l}0: 0 \\
0 \\
0 \\
0 \\
0 \\
0\end{array}$ & 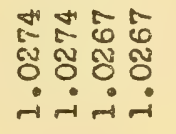 & & $\begin{array}{l}\infty \\
0 \\
0 \\
0 \\
0 \\
-i\end{array}$ & 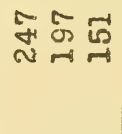 \\
\hline & 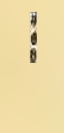 & $\begin{array}{lll}1 & 0 \\
\infty & \infty \\
0 & \infty \\
0 & \\
0 & \end{array}$ & 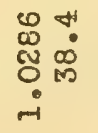 & 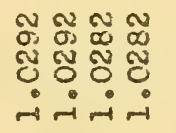 & & 1 & 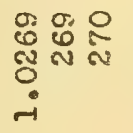 & 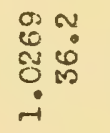 & 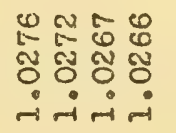 & & i & 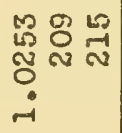 \\
\hline & i & $\begin{array}{ll}+1 & w \\
\infty & \infty \\
0 & 0 \\
0 & N \\
0 & \end{array}$ & $\begin{array}{l}\text { H } \\
\infty \\
0 \\
0 \\
0 \\
0 \\
0\end{array}$ & 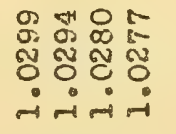 & & i & 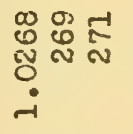 & $\begin{array}{l}\infty \\
0 \\
0 \\
0 \\
0 \\
-1\end{array}$ & 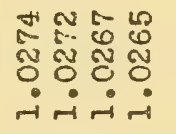 & & $i$ & 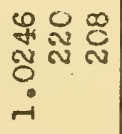 \\
\hline & i & $\begin{array}{l}+ \\
\infty \\
\infty \\
心 \\
0 \\
0\end{array}$ & $\begin{array}{ll}\infty & 0 \\
\infty & 0 \\
N & \infty \\
0 & m \\
: & \\
-1\end{array}$ & 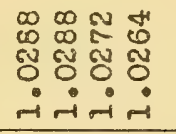 & & i & 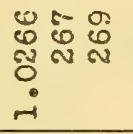 & $\begin{array}{l}5 \\
6 \\
0 \\
0 \\
0 \\
0 \\
i\end{array}$ & 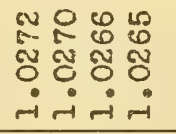 & & i & 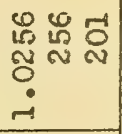 \\
\hline & $\begin{array}{l}0 \\
\text { O } \\
\text { - } \\
-1\end{array}$ & 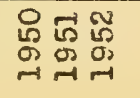 & & & & $\begin{array}{l}0 \\
\text { स } \\
0 \\
\rightarrow\end{array}$ & 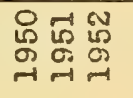 & & & & \begin{tabular}{l}
$\sigma$ \\
\multirow{2}{*}{} \\
-1
\end{tabular} & 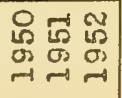 \\
\hline
\end{tabular}




\begin{tabular}{|c|c|c|c|c|c|c|c|c|c|c|}
\hline 蓠 & & & 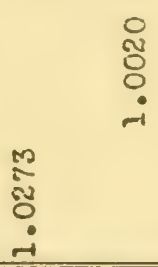 & & 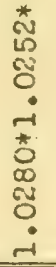 & 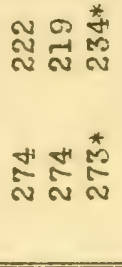 & & $\begin{array}{l}\circ \\
\approx \\
\text { Õ } \\
\dot{-1} \\
\end{array}$ & & 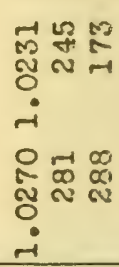 \\
\hline 莺 & & $\begin{array}{l}5 \infty \\
\sigma \\
10 \\
0 \\
\dot{0}\end{array}$ & & & i & 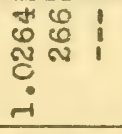 & $\begin{array}{ll}0 & \infty \\
4 & 0 \\
0 & 0 \\
0 & 0 \\
-1 & \end{array}$ & & & 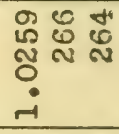 \\
\hline 巳் & & $\begin{array}{l}\sim \\
-1 \\
\tilde{O} \\
0 \\
-1\end{array}$ & 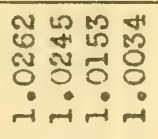 & & 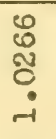 & 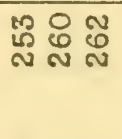 & $\begin{array}{ll}0 & 0 \\
0 & 0 \\
N & \text { N } \\
0 & \text { N } \\
-1\end{array}$ & 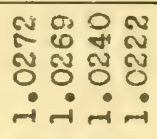 & & 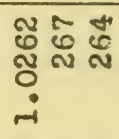 \\
\hline : & $\stackrel{0}{\stackrel{0}{2}}$ & 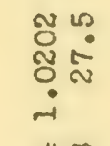 & 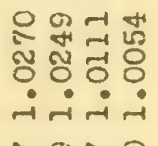 & & 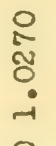 & 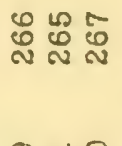 & $\begin{array}{l}5 \\
0 \\
0 \\
0 \\
0 \\
0\end{array}$ & 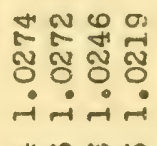 & & 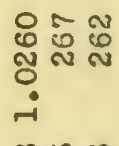 \\
\hline ثं & $\begin{array}{l}\text { Fृ } \\
\text { O } \\
\dot{0}\end{array}$ & $\begin{array}{l}4 \\
5 \\
0 \\
0\end{array}$ & 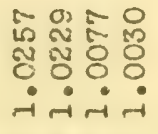 & $\frac{u}{\bar{m}}$ & $\begin{array}{l}0 \\
2 \\
8 \\
0 \\
-1\end{array}$ & 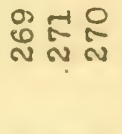 & 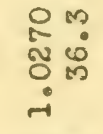 & 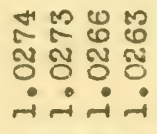 & & 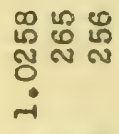 \\
\hline 莒 & $\begin{array}{l}\frac{1}{3} \\
\frac{0}{2} \\
0\end{array}$ & $\begin{array}{l}0 \\
0 \\
-10 \\
0 \\
-1\end{array}$ & 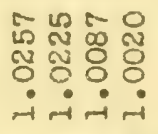 & $\begin{array}{l}\frac{a}{a} \\
\frac{u}{\alpha} \\
z\end{array}$ & \begin{tabular}{l}
0 \\
\multirow{2}{*}{} \\
0 \\
-1
\end{tabular} & 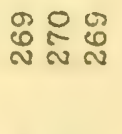 & \begin{tabular}{l}
0 \\
\multirow{2}{*}{} \\
0 \\
0 \\
0
\end{tabular} & 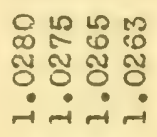 & $\frac{1}{\infty}$ & 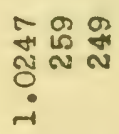 \\
\hline 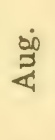 & $\frac{u}{z}$ & $\begin{array}{l}0 \\
\infty \\
0 \\
-1 \\
0 \\
-1\end{array}$ & 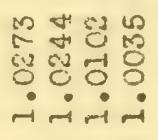 & $\frac{\frac{\pi}{2}}{2}$ & 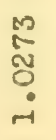 & 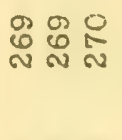 & 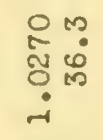 & 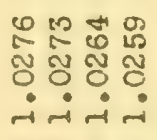 & 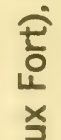 & 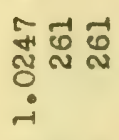 \\
\hline$\frac{2}{3}$ & 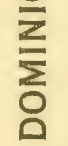 & $\begin{array}{l}n \infty \\
0 \\
0 \\
0 \\
0 \\
-1\end{array}$ & 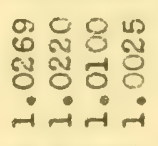 & อ & \begin{tabular}{l}
\multirow{2}{\tilde{N}}{} \\
0 \\
-
\end{tabular} & $\begin{array}{lll}\infty & \infty & 0 \\
0 & 0 & 0 \\
\sim & \sim & \sim\end{array}$ & 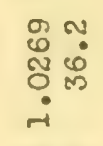 & 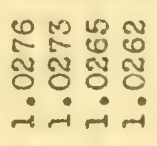 & $\sum_{\frac{1}{1}}^{2}$ & 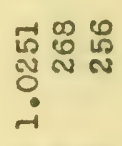 \\
\hline$\underset{\Xi}{\Xi}$ & 0 & $\begin{array}{ll}n & 0 \\
\infty & 0 \\
-1 & \text { in } \\
0 & 0\end{array}$ & 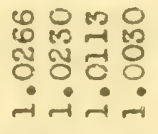 & $\frac{5}{a}$ & $\begin{array}{c}5 \\
5 \\
0 \\
0 \\
-1\end{array}$ & 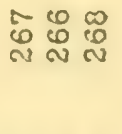 & $\begin{array}{ll}\infty & 0 \\
0 & 0 \\
\widetilde{O} & 0 \\
0 & :-1 \\
-1\end{array}$ & $\begin{array}{lll}0 & 0 & 0 \\
0 & 0 & 0 \\
0 & 1 & 0 \\
0 & 0 & 0 \\
0 & 0 & 0 \\
-17-1 & -1\end{array}$ & $\frac{3}{3}$ & 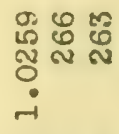 \\
\hline 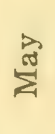 & $\frac{2}{2}$ & $\begin{array}{l}0 \\
0 \\
0\end{array}$ & 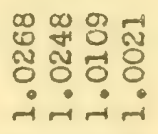 & $\frac{\alpha}{\frac{\alpha}{2}}$ & $\frac{1}{N}$ & 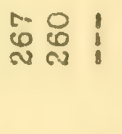 & $\begin{array}{ll}0 & \infty \\
0 & \dot{0} \\
0 & \text { i } \\
0 & \text { N } \\
-i\end{array}$ & 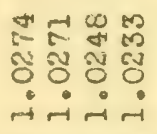 & & 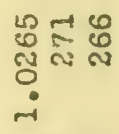 \\
\hline 客 & $\frac{5}{0}$ & $\begin{array}{ll} & \sigma \\
-1 & 0 \\
\tilde{O} & 0 \\
0 & 0\end{array}$ & 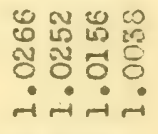 & & $i$ & 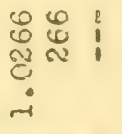 & $\begin{array}{l}0 \\
0 \\
0 \\
0 \\
0 \\
0\end{array}$ & 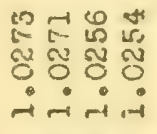 & & 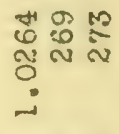 \\
\hline$\sum^{\stackrel{5}{ \pm}}$ & & 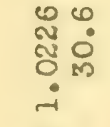 & 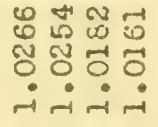 & & $i$ & $\begin{array}{lcl}-1 & \infty & 1 \\
6 & 6 & 1 \\
0 & \infty & 1 \\
0 & \end{array}$ & $\begin{array}{l}\text { H } \\
0 \\
0 \\
0 \\
0 \\
\text { i } \\
\text { i }\end{array}$ & 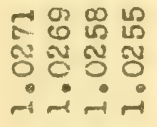 & & 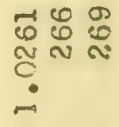 \\
\hline 远 & & 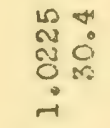 & 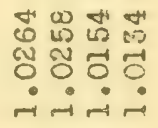 & & i & $\begin{array}{lll}0 & 10 & 1 \\
10 & 0 & 1 \\
0 & 3 & 1 \\
0 & \end{array}$ & 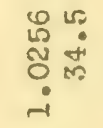 & 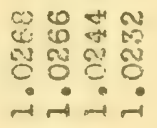 & & 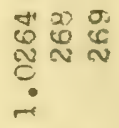 \\
\hline 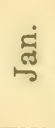 & & 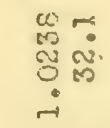 & 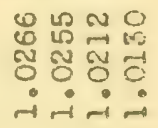 & & 1 & $\begin{array}{lll}0 & 0 & 0 \\
0 & 0 & 0 \\
N & v & 0 \\
0 & \end{array}$ & 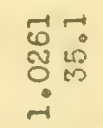 & 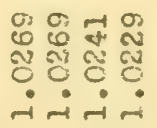 & & 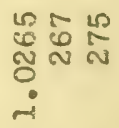 \\
\hline ङ & & 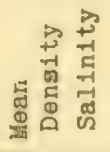 & 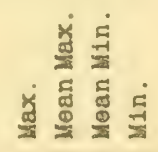 & & 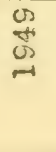 & 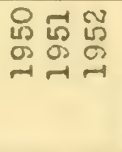 & 究 & 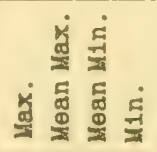 & & 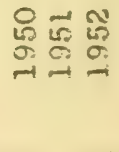 \\
\hline
\end{tabular}




\begin{tabular}{|c|c|c|c|c|c|c|c|c|c|c|c|}
\hline & $\begin{array}{l}\infty \\
\infty \\
\infty \\
\text { N1 } \\
0 \\
-1 \\
\end{array}$ & & $\begin{array}{l}\vdots \\
\vdots \\
* \\
\tilde{O} \\
0 \\
\vdots \\
-1 \\
\end{array}$ & 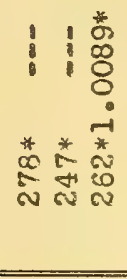 & & $\begin{array}{c}10 \\
0 \\
0 \\
0 \\
0 \\
-1 \\
\end{array}$ & & 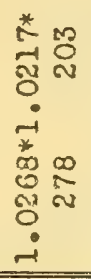 & 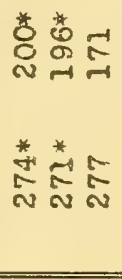 & & 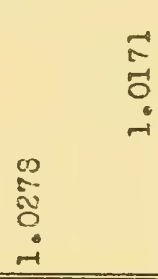 \\
\hline $\begin{array}{ll}m & 4 \\
0 & 0 \\
0 & 0 \\
0 & 0 \\
0\end{array}$ & & & $i$ & 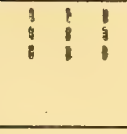 & $\begin{array}{l}m \\
0 \\
0 \\
0 \\
0\end{array}$ & & & 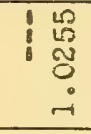 & $i \mid \begin{array}{c}n \\
\text { in } \\
\text { in }\end{array}$ & 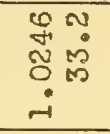 & \\
\hline 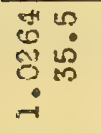 & 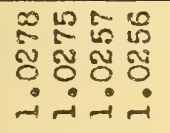 & & $\begin{array}{l}\infty \\
\infty \\
0 \\
0 \\
0 \\
-1\end{array}$ & $\begin{array}{l}10 \\
1 \\
1\end{array}$ & $\begin{array}{l}\sigma_{0}^{\infty} \\
\sigma_{0}^{\circ} \\
\dot{\sim}\end{array}$ & 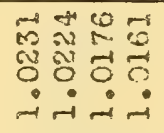 & & 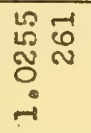 & సิ & 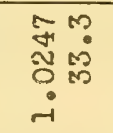 & 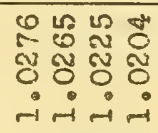 \\
\hline $\begin{array}{l}0 \\
0 \\
0 \\
0 \\
0 \\
0 \\
-i\end{array}$ & 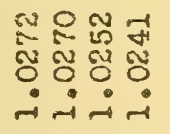 & & $\begin{array}{l}5 \\
\infty \\
0 \\
-i\end{array}$ & 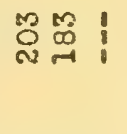 & $\begin{array}{l}-0 \\
0 \\
0 \\
0\end{array}$ & 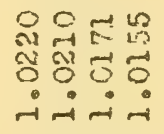 & & 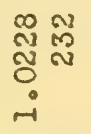 & $\begin{array}{l:l}m & m \\
-1 & M\end{array}$ & $\begin{array}{ll}0 & c \\
N & 0 \\
N & 0 \\
0 & m \\
i & \end{array}$ & 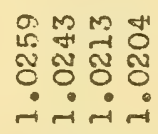 \\
\hline 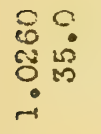 & 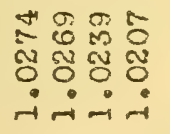 & $\div$ & $\begin{array}{l}0 \\
0 \\
0 \\
0 \\
-1\end{array}$ & 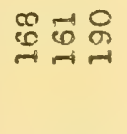 & 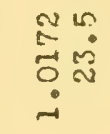 & 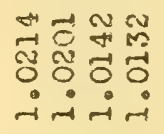 & & $\begin{array}{l}\infty \\
\mathrm{N} \\
\mathrm{N} \\
0 \\
-1\end{array}$ & 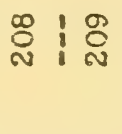 & 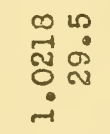 & 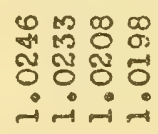 \\
\hline 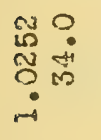 & 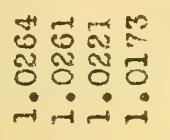 & $\begin{array}{l}3 \\
i\end{array}$ & $\begin{array}{l}0 \\
10 \\
0 \\
0 \\
0\end{array}$ & ली & $\begin{array}{l}m ? \\
\text { in } \\
0 \\
0\end{array}$ & 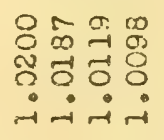 & $\frac{\pi}{\sum}$ & \begin{tabular}{l}
0 \\
1 \\
\multirow{2}{*}{} \\
0 \\
-1
\end{tabular} & $\stackrel{O}{\stackrel{O}{N}}: \frac{0}{N}$ & 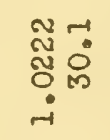 & 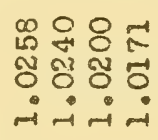 \\
\hline 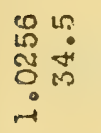 & 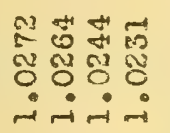 & $\begin{array}{l}\pi \\
0 \\
0 \\
0 \\
0 \\
0\end{array}$ & $\begin{array}{l}0 \\
12 \\
\stackrel{0}{0} \\
-i\end{array}$ & 零出嵒 & $\begin{array}{l}\begin{array}{l}3 \\
+\end{array} \\
\dot{0} \\
0 \\
-1\end{array}$ & 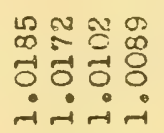 & $\begin{array}{l}\frac{5}{0} \\
0 \\
0\end{array}$ & i & 尔: & $\begin{array}{l}\infty \\
m \\
\tilde{N} \\
0 \\
0 \\
-1\end{array}$ & 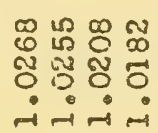 \\
\hline 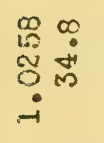 & 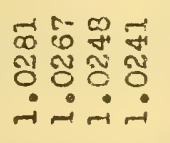 & $\underbrace{\frac{1}{d}}_{0}$ & $\begin{array}{l}\infty \\
\infty \\
\overrightarrow{0} \\
-i\end{array}$ & 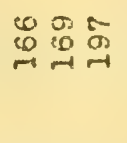 & $\begin{array}{l}0 \\
0 \\
0 \\
0 \\
0 \\
0 \\
-1\end{array}$ & 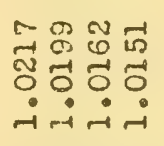 & $\frac{x_{0}^{2}}{0}$ & $\begin{array}{l}0 \\
10 \\
0 \\
0 \\
0 \\
-1\end{array}$ & స: & $\begin{array}{l}\text { mo } \\
\text { al } \\
0 \\
i \\
i\end{array}$ & 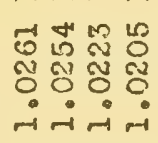 \\
\hline 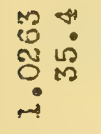 & 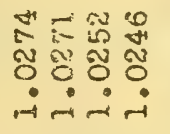 & $\frac{2}{2}$ & i & 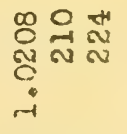 & $\begin{array}{l}40 \\
\vec{H} \\
0 \\
0 \\
0 \\
-1\end{array}$ & 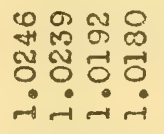 & $\frac{b}{0}$ & 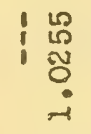 & 货赑 & 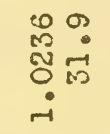 & 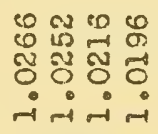 \\
\hline 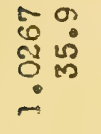 & 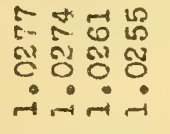 & $F$ & $\vdots$ & $\begin{array}{l:l}\text { in } \\
\text { in } \\
0 \\
0 \\
0\end{array}$ & 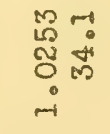 & 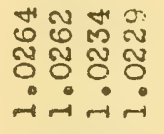 & & $\begin{array}{l}0 \\
0 \\
10 \\
0 \\
0\end{array}$ & | 연 & 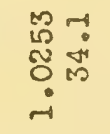 & 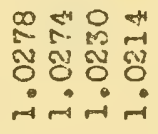 \\
\hline $\begin{array}{l}80 \\
6 \\
0 \\
0 \\
0 \\
01\end{array}$ & 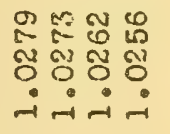 & & & 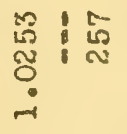 & $\begin{array}{l}\pi \\
0 \\
0 \\
0\end{array}$ & 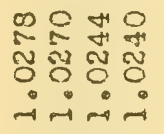 & & 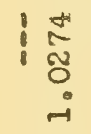 & 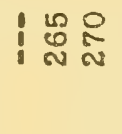 & 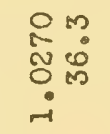 & 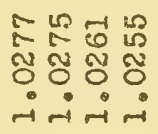 \\
\hline 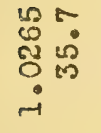 & 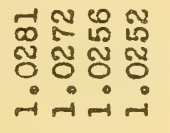 & & i & 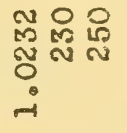 & $\begin{array}{l}1 \\
\text { Mิ } \\
0 \\
0 \\
-1\end{array}$ & 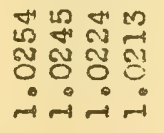 & & 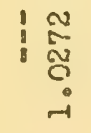 & i: : & 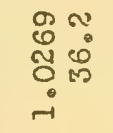 & 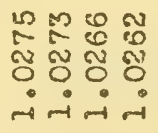 \\
\hline 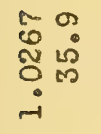 & 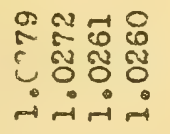 & & 8 & 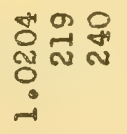 & 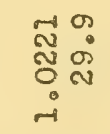 & 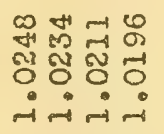 & & $\begin{array}{l}\text { i. } \\
1 \text { N } \\
\text { i } \\
\text { i }\end{array}$ & 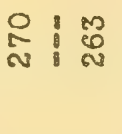 & $\begin{array}{ll}\infty & 0 \\
0 & 0 \\
N & 0 \\
0 & m \\
i & \end{array}$ & 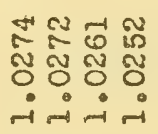 \\
\hline 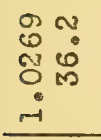 & 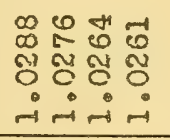 & & 8 & 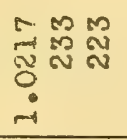 & $\begin{array}{l}\text { M } \\
\text { N } \\
0 \\
0 \\
0 \\
-1\end{array}$ & 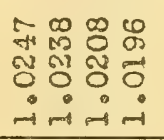 & & $\begin{array}{l}5 \\
0 \\
0 \\
0 \\
-1\end{array}$ & 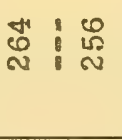 & 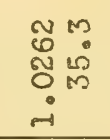 & 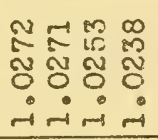 \\
\hline 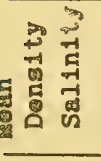 & 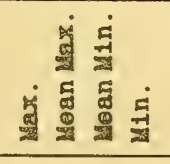 & & $\underset{\infty}{\infty}$ & 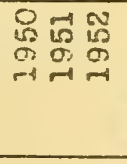 & 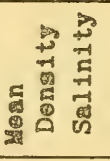 & 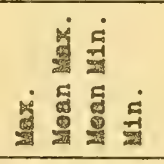 & & 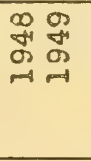 & 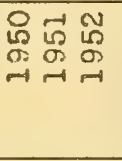 & 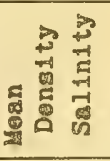 & 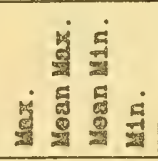 \\
\hline
\end{tabular}




\begin{tabular}{|c|c|c|c|c|c|c|c|c|c|c|}
\hline 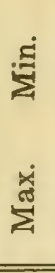 & & 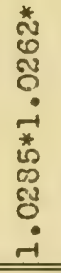 & 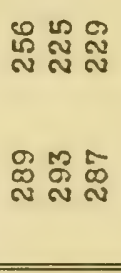 & & 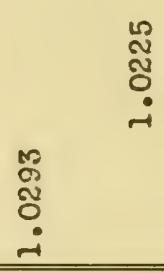 & & 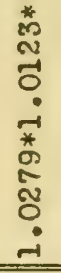 & 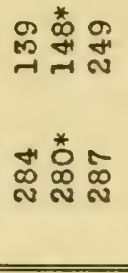 & & 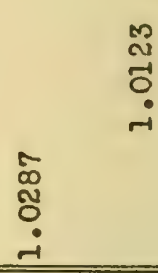 \\
\hline 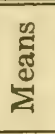 & & i & 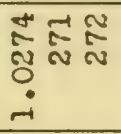 & 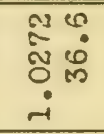 & & & i & 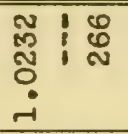 & 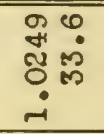 & \\
\hline 巳் & & $\begin{array}{l}8 \\
\infty \\
0 \\
-1\end{array}$ & 过思 & $\begin{array}{l}0 m \\
0 \\
0 \\
0 \\
0\end{array}$ & 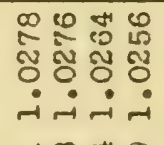 & & $\begin{array}{c}\text { Na } \\
\text { Oั } \\
-i\end{array}$ & 됴워 & $\begin{array}{l}\infty \\
\tilde{n} \\
\tilde{0} \\
\dot{-1}\end{array}$ & 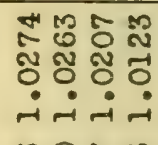 \\
\hline z & & 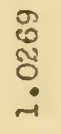 & 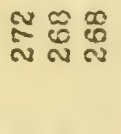 & $\begin{array}{l}0 \\
0 \\
0 \\
0 \\
0 \\
-1\end{array}$ & 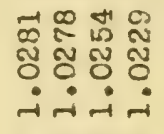 & & $\begin{array}{l}\infty \\
6 \\
0 \\
0 \\
-\end{array}$ & 芯忒芯 & 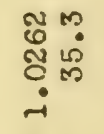 & 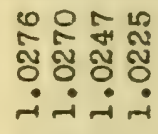 \\
\hline$\dot{0}$ & & $\begin{array}{l}\stackrel{\tilde{L}}{\tilde{Z}} \\
\stackrel{-i}{0}\end{array}$ & 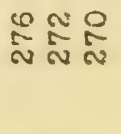 & 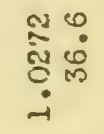 & 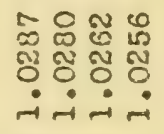 & & 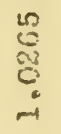 & 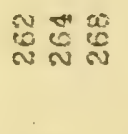 & $\begin{array}{l}0 \\
0 \\
0 \\
0 \\
0 \\
0\end{array}$ & 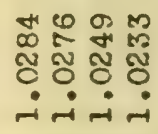 \\
\hline कें & 峁 & $\begin{array}{l}\infty \\
\stackrel{0}{\tilde{N}} \\
-i\end{array}$ & 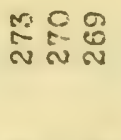 & 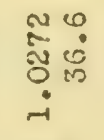 & 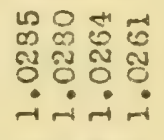 & 峞 & $i$ & 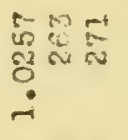 & $\begin{array}{l}4 \text { in } \\
5 \\
5 \\
0 \\
0 \\
i\end{array}$ & 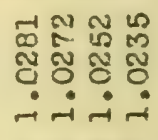 \\
\hline$\stackrel{3}{3}$ & $\frac{N}{2}$ & i & $\begin{array}{l}\text { in } \\
\tilde{\sigma} \\
\tilde{0}\end{array}$ & \begin{tabular}{l}
25 \\
\multirow{2}{*}{$:$} \\
0 \\
0
\end{tabular} & 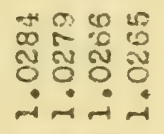 & $\begin{array}{l}N \\
\text { u } \\
>\end{array}$ & $i$ & 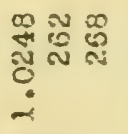 & 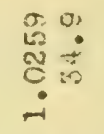 & 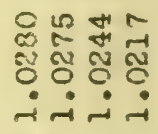 \\
\hline$\stackrel{3}{3}$ & $\frac{\pi}{a}$ & i & 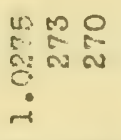 & 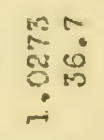 & 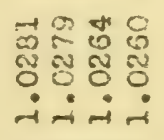 & $\underbrace{0}_{\substack{0 \\
\sum}}$ & $i$ & 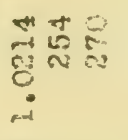 & نं & 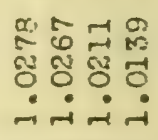 \\
\hline$\stackrel{\Xi}{\Xi}$ & 5 & i & $\begin{array}{l}c \\
0 \\
0 \\
0\end{array}$ & $\begin{array}{l}05 \\
05 \\
05\end{array}$ & 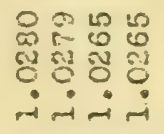 & $\frac{\pi}{0}$ & $i$ & 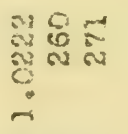 & $\begin{array}{l}m \\
i \\
6 \\
0\end{array}$ & 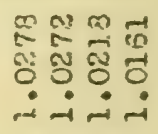 \\
\hline$\sum_{i}^{\infty}$ & & $i$ & $\begin{array}{l}m \\
0 \\
0 \\
m\end{array}$ & 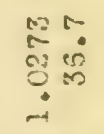 & 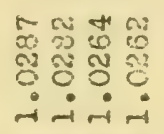 & & 1 & 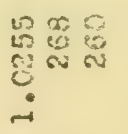 & $\begin{array}{ll}20 & -1 \\
50 & 0 \\
0 & 0\end{array}$ & 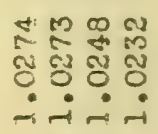 \\
\hline$\frac{\dot{a}}{4}$ & & i & 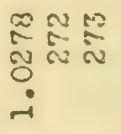 & 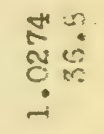 & 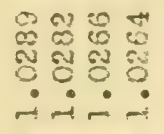 & & i & 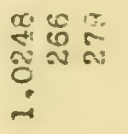 & $\begin{array}{l}0 \\
0 \\
0 \\
0 \\
0 \\
0\end{array}$ & 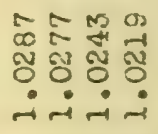 \\
\hline$\sum_{i}^{\infty}$ & & i & 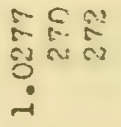 & 范 & 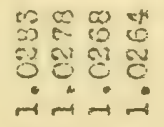 & & $i$ & 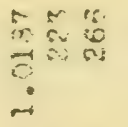 & 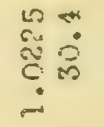 & 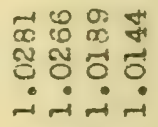 \\
\hline$\frac{0}{10}$ & & $i$ & 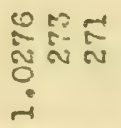 & 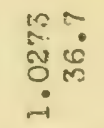 & 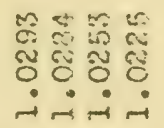 & & $i$ & $\begin{array}{l}0 \\
\sigma \\
\sigma \\
0 \\
0\end{array}$ & 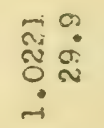 & 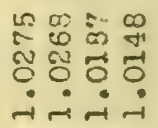 \\
\hline 宛 & & ! & 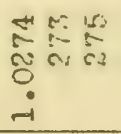 & $\begin{array}{l}+\infty \\
\stackrel{5}{\infty} \infty \\
0 \\
0 \\
0\end{array}$ & 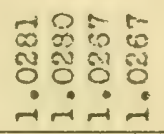 & & ! & $\begin{array}{lll}\because & 1 & c \\
\vdots & 1 & 6 \\
\vdots & \end{array}$ & 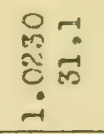 & 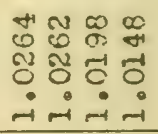 \\
\hline 氜 & & $\begin{array}{l}0 \\
0 \\
9 \\
2 \\
-4\end{array}$ & 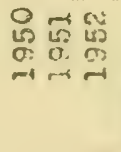 & 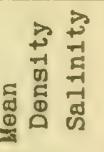 & 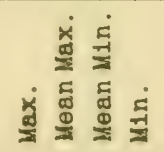 & & $\begin{array}{l}m \\
\text { g } \\
\text { m }\end{array}$ & 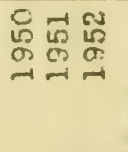 & 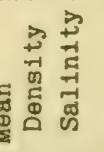 & 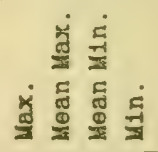 \\
\hline
\end{tabular}




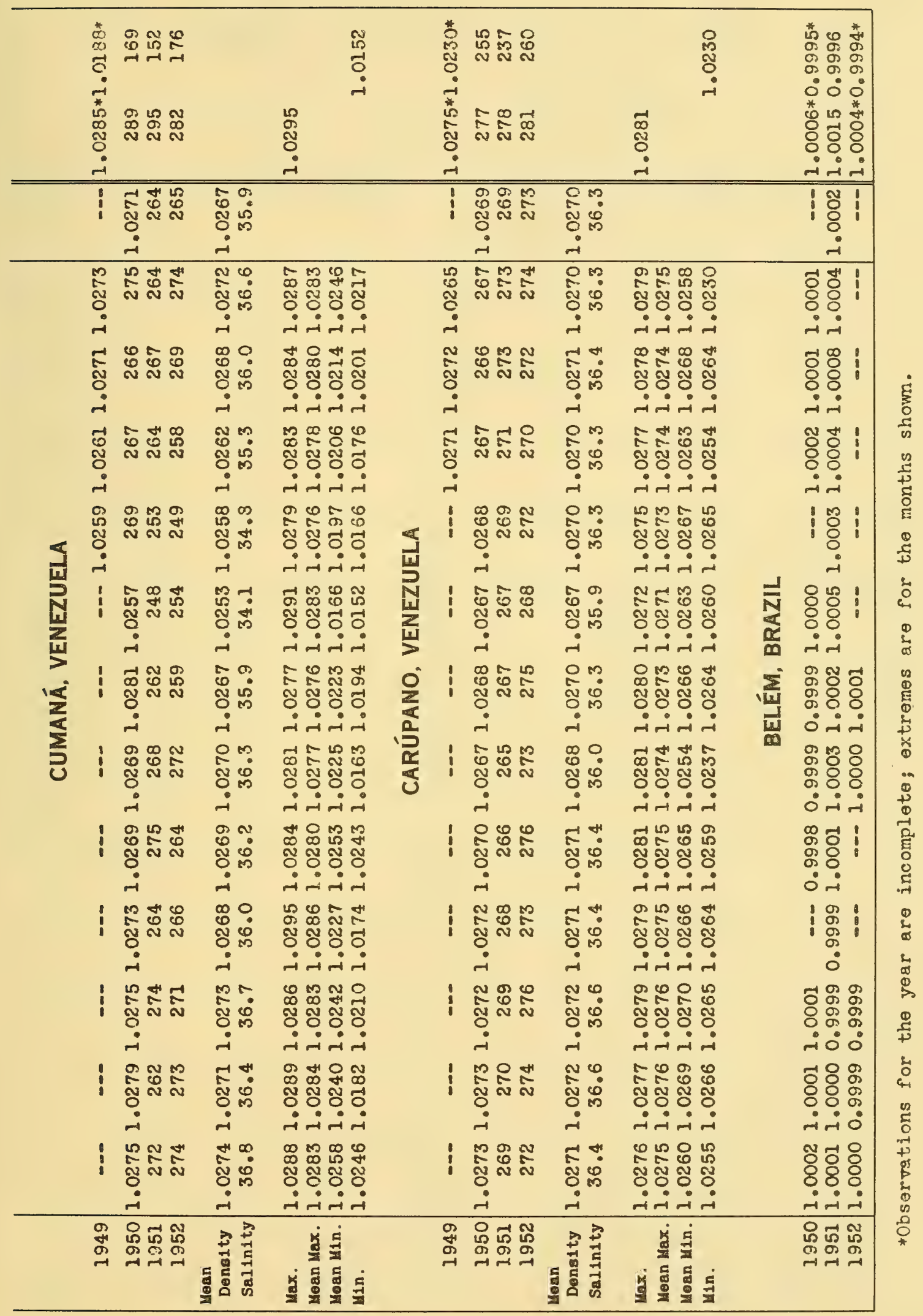




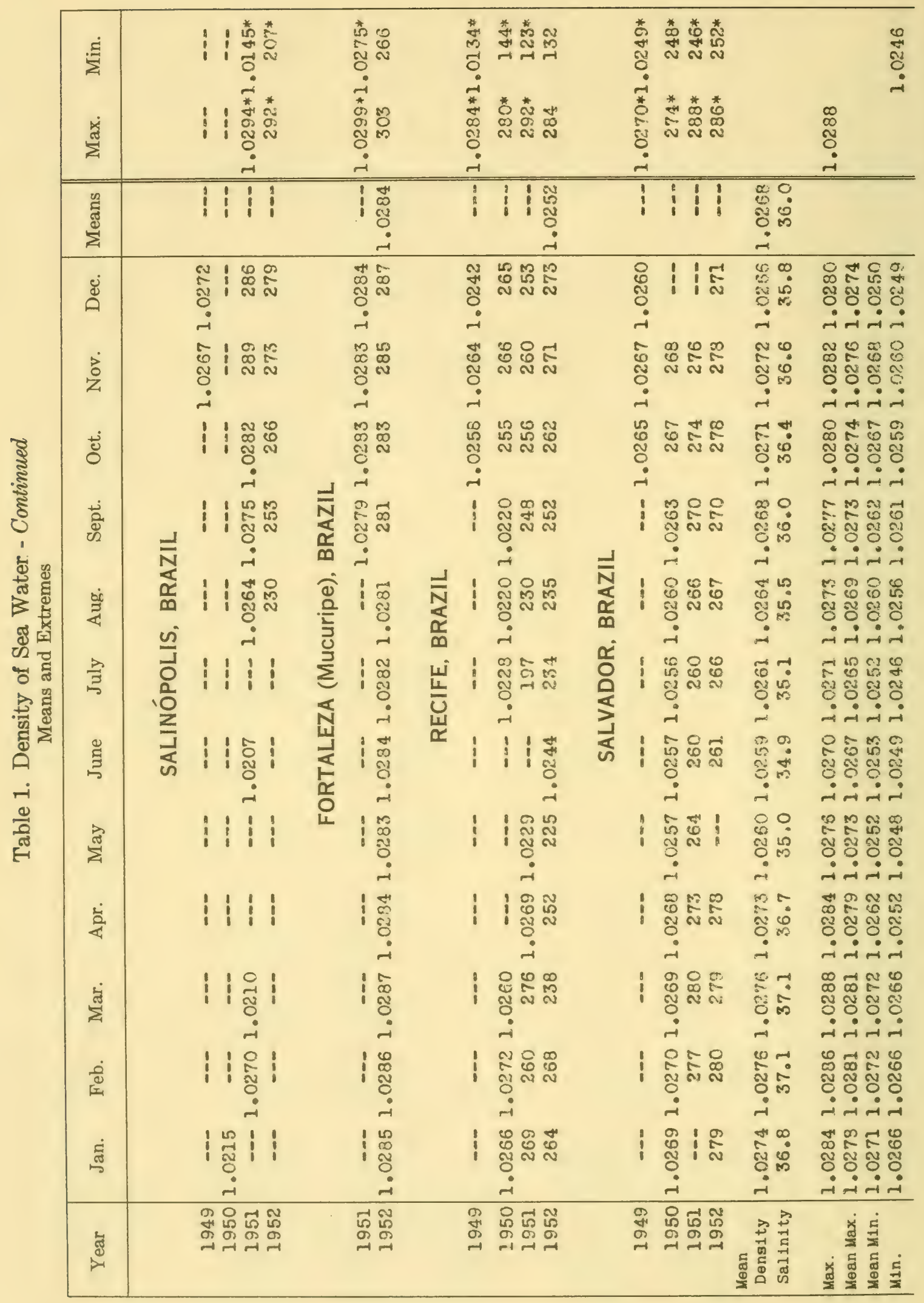




\begin{tabular}{|c|c|c|c|c|c|c|c|c|c|c|c|}
\hline & 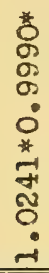 & & 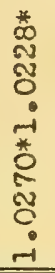 & 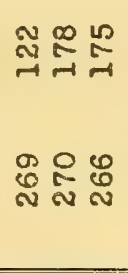 & & $\begin{array}{l}\text { N } \\
\text { - } \\
\dot{-1} \\
-1\end{array}$ & & 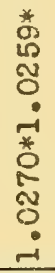 & 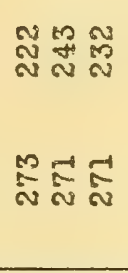 & & 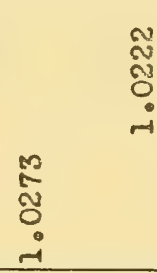 \\
\hline & $i$ & & $i$ & 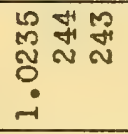 & 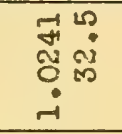 & & & i & 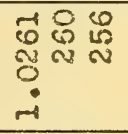 & $\begin{array}{l}9 \\
10 \\
0 \\
0 \\
-1 \\
-1\end{array}$ & \\
\hline \multirow{13}{*}{ 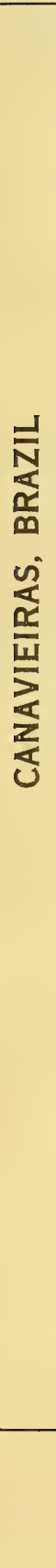 } & $\begin{array}{l}10 \\
8 \\
8 \\
0\end{array}$ & \multirow{5}{*}{ 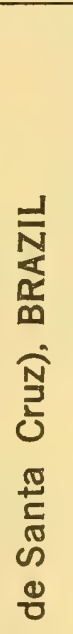 } & $\begin{array}{l}\text { in } \\
\text { ơ } \\
0 \\
-1\end{array}$ & $\begin{array}{l}\text { W } \\
\text { 心 } \\
\text { 心 } \\
\text { 心 }\end{array}$ & 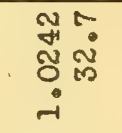 & 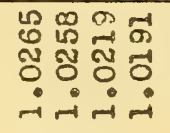 & & $\begin{array}{l}0 \\
\vdots \\
0 \\
0 \\
-1\end{array}$ & 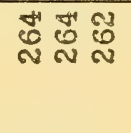 & 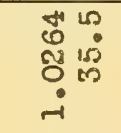 & 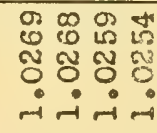 \\
\hline & 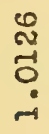 & & \begin{tabular}{l}
0 \\
\multirow{H}{*}{} \\
0 \\
-1
\end{tabular} & 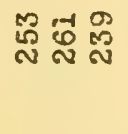 & 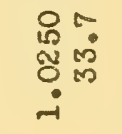 & 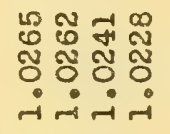 & & 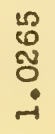 & 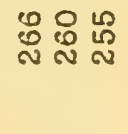 & 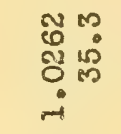 & 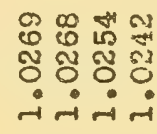 \\
\hline & 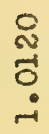 & & $\begin{array}{l}\sharp \\
0 \\
\text { Oे } \\
-1\end{array}$ & 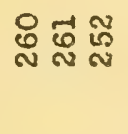 & 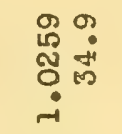 & 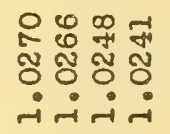 & & 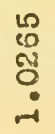 & 品恕 & 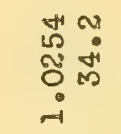 & 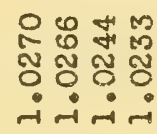 \\
\hline & $\begin{array}{l}\infty \\
0 \\
-1 \\
0 \\
-1\end{array}$ & & ! & 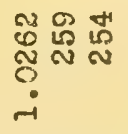 & 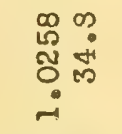 & 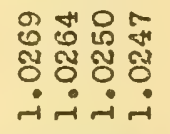 & $=$ & i & 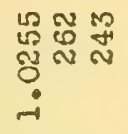 & 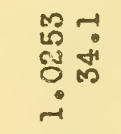 & 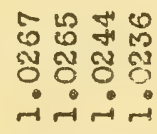 \\
\hline & $\begin{array}{l}\infty \\
\infty \\
8 \\
0 \\
-1\end{array}$ & & i & 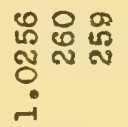 & 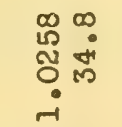 & 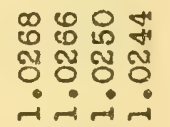 & $\frac{\mathbb{N}}{\frac{1}{m}}$ & i & 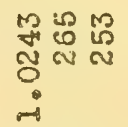 & 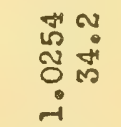 & 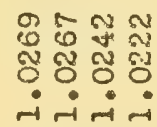 \\
\hline & $\begin{array}{l}0 \\
\text { N } \\
\text {-1 } \\
0\end{array}$ & $\frac{N}{N}$ & ! & 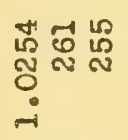 & 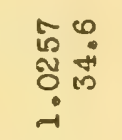 & 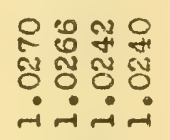 & $\frac{\mathbb{m}}{2}$ & i & 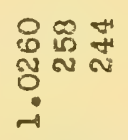 & 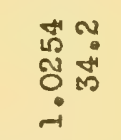 & 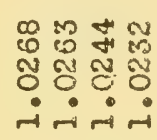 \\
\hline & $\begin{array}{l}5 \\
\delta \\
0 \\
0 \\
-1\end{array}$ & $\begin{array}{l}= \\
0 \\
0 \\
\text { 몬 }\end{array}$ & i & 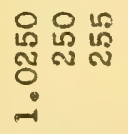 & 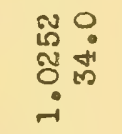 & 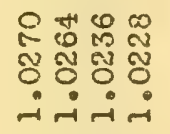 & $\Sigma$ & i & 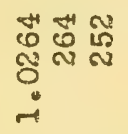 & 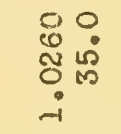 & 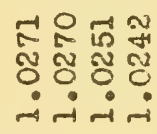 \\
\hline & $\begin{array}{l}0 \\
8 \\
0 \\
0 \\
-1\end{array}$ & $\sum_{\text {u }}^{2}$ & i & 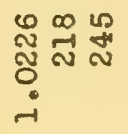 & 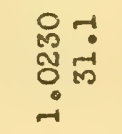 & 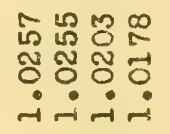 & & i & 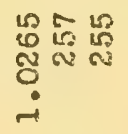 & 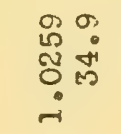 & 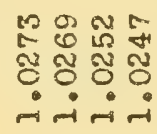 \\
\hline & $\begin{array}{l}10 \\
8 \\
0 \\
0 \\
-1\end{array}$ & $\frac{0}{\alpha x}$ & $i$ & 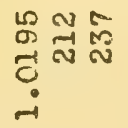 & 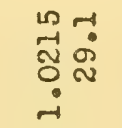 & 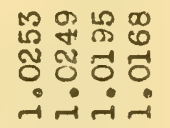 & & i & 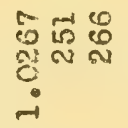 & \begin{tabular}{l}
$\vec{c}$ \\
\multirow{0}{0}{} \\
0 \\
$\dot{0}$
\end{tabular} & 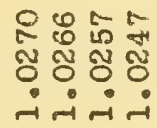 \\
\hline & $\begin{array}{l}\text { L } \\
0 \\
0 \\
0 \\
\text { i }\end{array}$ & & i & 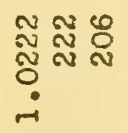 & $\begin{array}{l}n \\
\overrightarrow{0} \\
0 \\
0\end{array}$ & 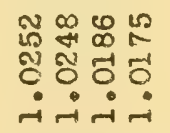 & & i & 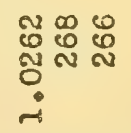 & 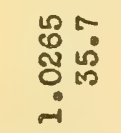 & 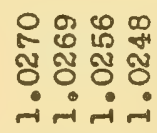 \\
\hline & $\begin{array}{l}0 \\
0 \\
0 \\
0\end{array}$ & & ! & 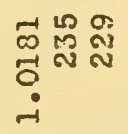 & 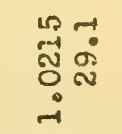 & 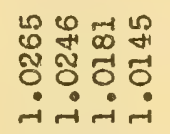 & & i & 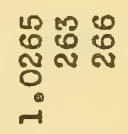 & $\begin{array}{l}\text { L } \\
0 \\
0 \\
0 \\
0 \\
0 \\
-1\end{array}$ & 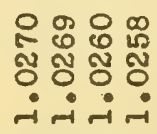 \\
\hline & $i$ & & $i$ & 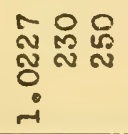 & 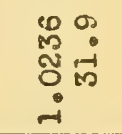 & 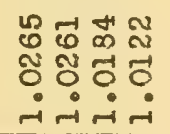 & & i & 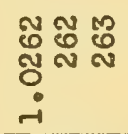 & 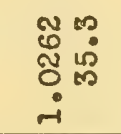 & 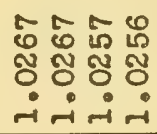 \\
\hline & 旅 & & \begin{tabular}{c}
9 \\
\multirow{2}{*}{} \\
-1
\end{tabular} & 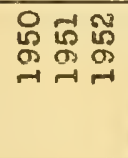 & 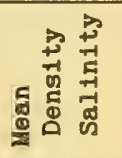 & 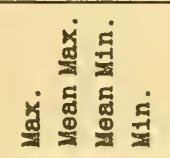 & & $\begin{array}{l}0 \\
\text { gै } \\
\text { r- } \\
\text { - }\end{array}$ & 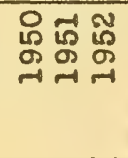 & 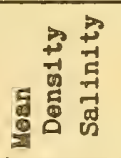 & 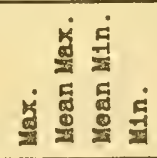 \\
\hline
\end{tabular}




\section{Mean Salinity Curves}

Monthly mean salinities in parts per thousand are presented graphically to show the seasonal variation.

IVIGTUT, GREENLAND 1945-1952

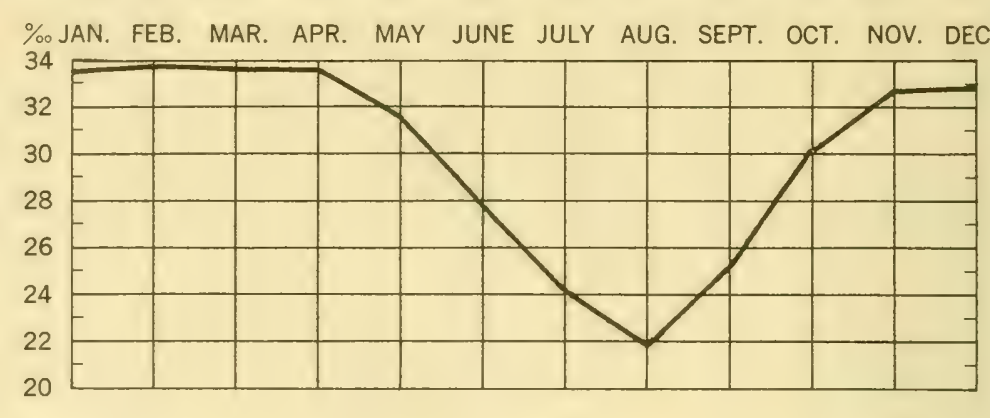

EASTPORT, ME. 1930-1952

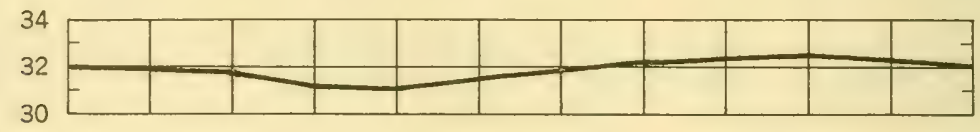

BAR HARBOR, ME. 1947-1952

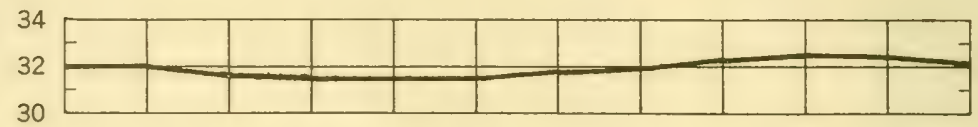

PORTLAND, ME. 1922-1945

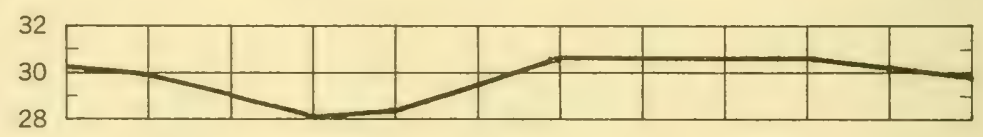

PORTSMOUTH, N.H. 1944-1952

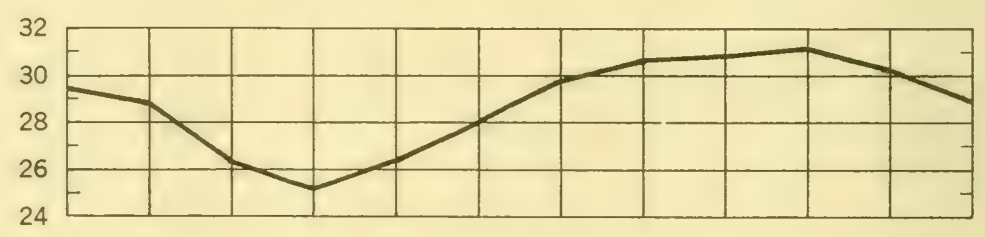

BOSTON, MASS. 1922-1952

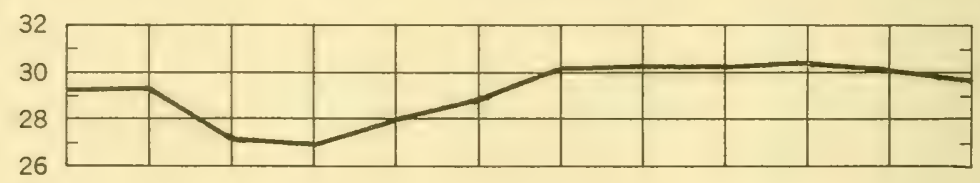

WOODS HOLE, MASS. 1944-1952

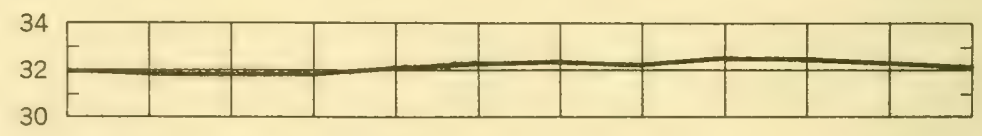

NEW LONDON. CONN. 1947-1952

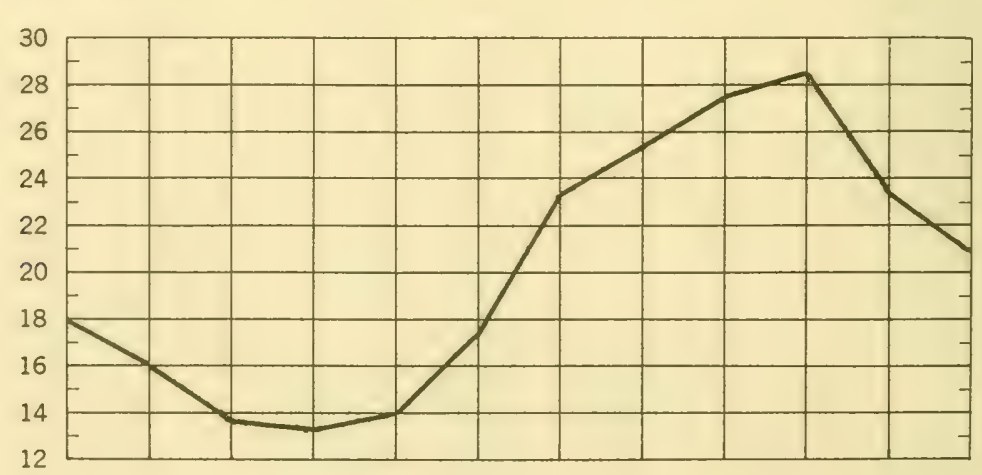


$\%$ JAN. FEB. MAR. APR. MAY JUNE JULY AUG. SEPT. OCT. NOV. DEC. FORT POND BAY, L.I., N.Y. 30 1947-1952

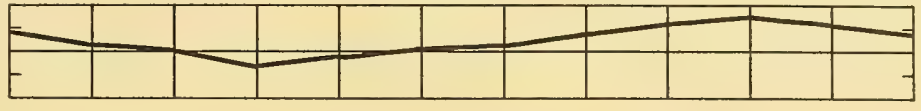

NEW YORK (Willets Point), N.Y. 1932-1952

NEW YORK

(The Battery), N.Y. 1927-1952

NEW YORK

(Fort Hamilton), N.Y. 1911-1920, 1927-1928

SANDY HOOK, N.J, 1887-1893, 1944-1952

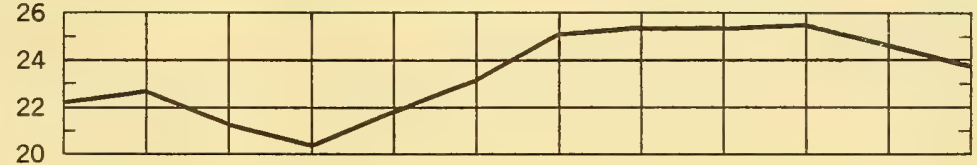

ATLANTIC CITY, N.J. 1912-1920, 1923-1952

PHILADELPHIA, PA. 1923-1946

CAPE CHARLES (town), VA. 1947.1952

32 30
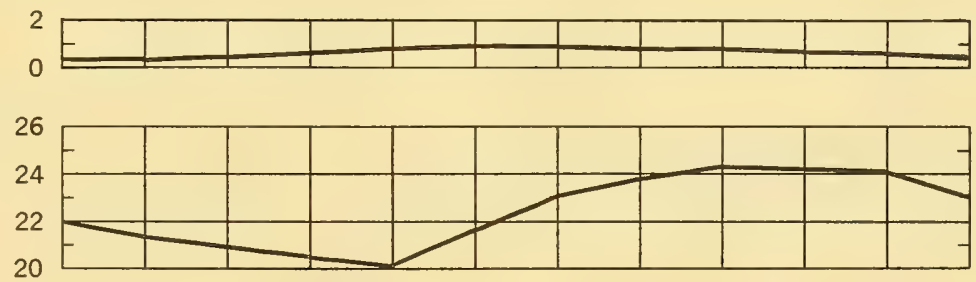

OLD POINT COMFORT, V.A. 20 1943-1945, 1948-1952

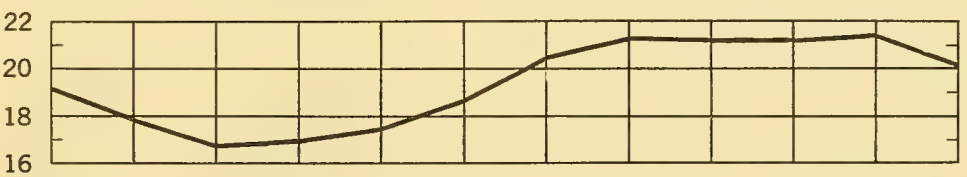

RICHMOND, VA. 1947-1952

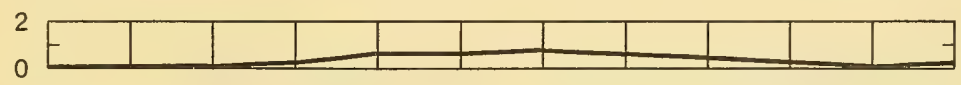

WASHINGTON, D.C. 1944-1952

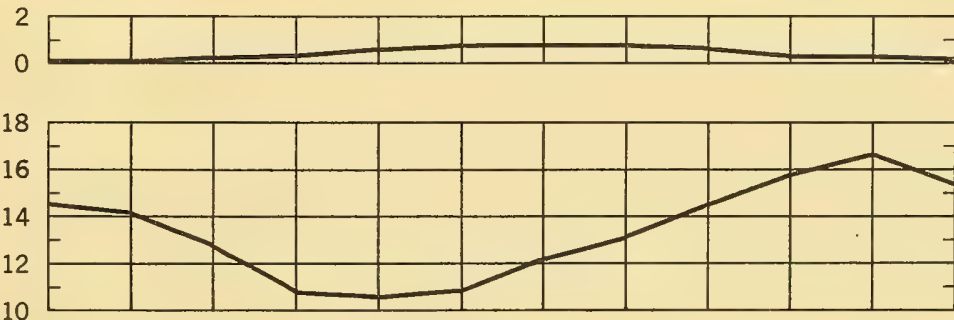


CAMBRIDGE, MD.

1945-1952

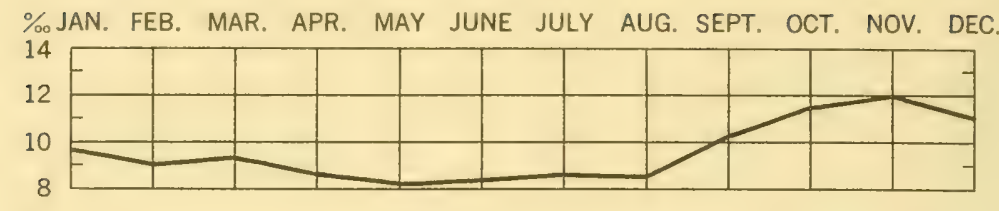

ANNAPOLIS, MD.

1947-1952

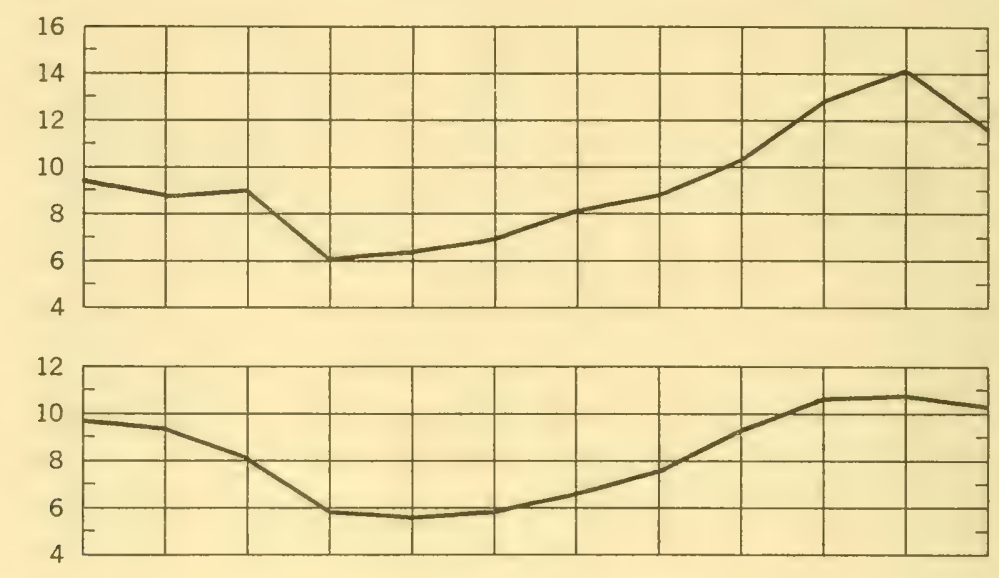

DIAMOND SHOAL LIGHTSHIP 36 1923-1928

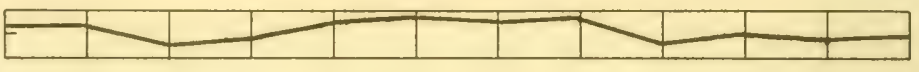

SOUTHPORT, N.C. 1946-1952

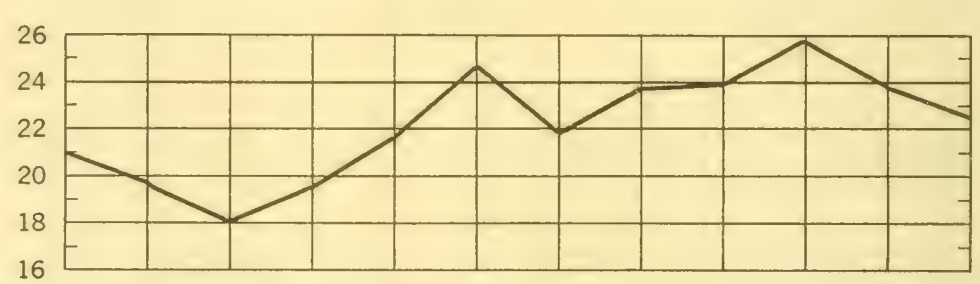

CHARLESTON, S.C. 1922-1942

See footnote $(\dagger)$ on page 31 .

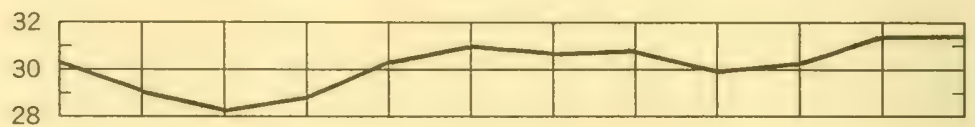

CHARLESTON, S.C. $1942-1952$

See footnote $(\dagger)$ on page 31 .

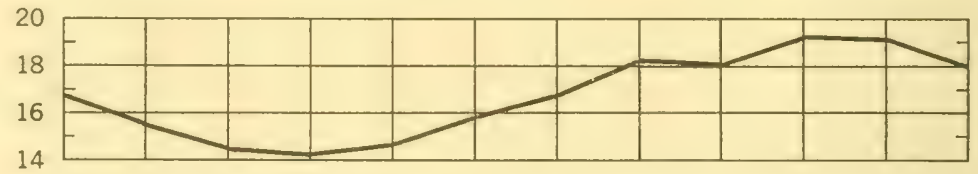

FORT PULASKI, GA. 1940-1952

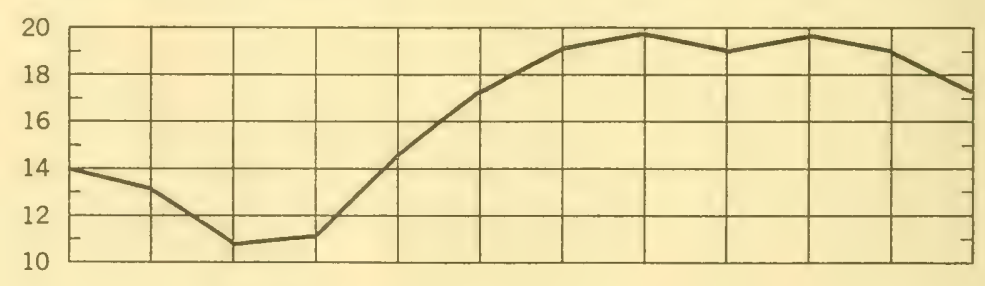

FERNANDINA, FLA. 1944-1952

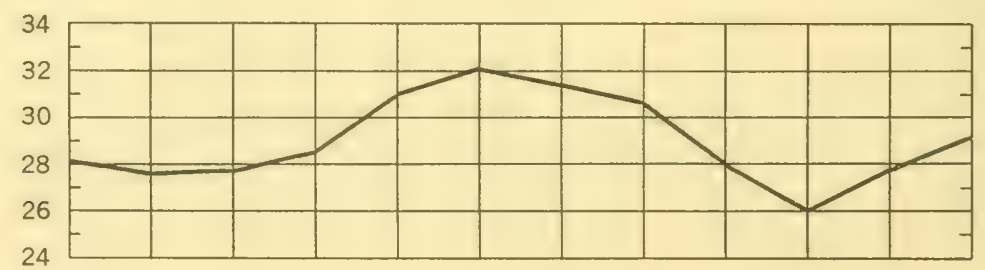


MAYPORT, FLA. 1945-1952

$\%$ JAN. FEB. MAR. APR. MAY JUNE JULY AUG. SEPT. OCT. NOV. DEC.

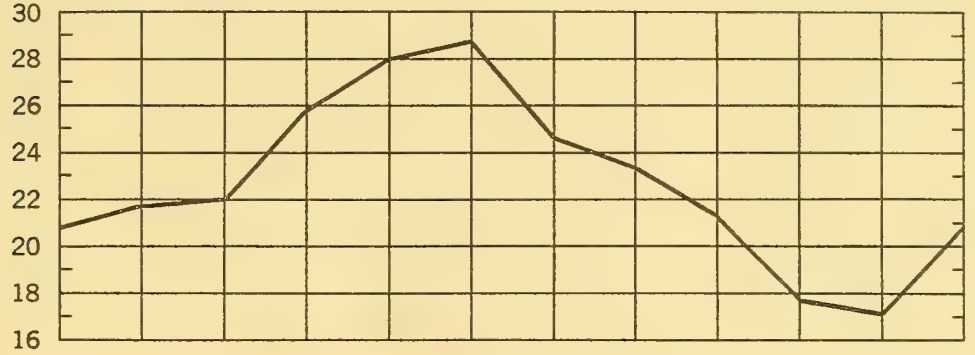

DAYTONA BEACH (ocean), FLA.

1925-1932, 1939-1950

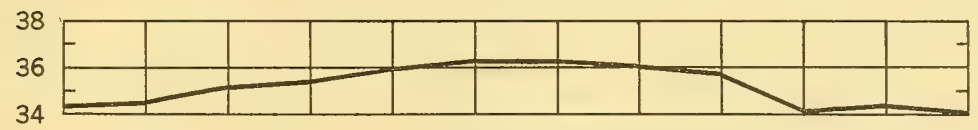

MIAMI BEACH, FLA. 1940-1951

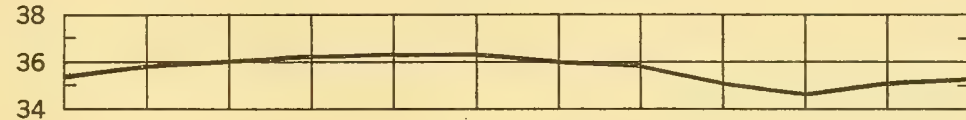

KEY WEST, FLA. 1926-1940, 1946-1952

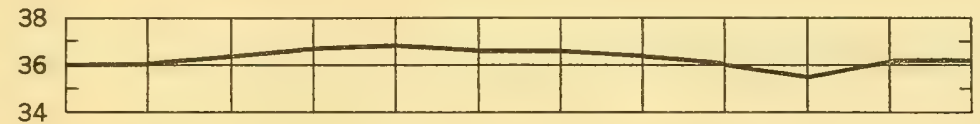

ST. PETERSBURG, FLA. 1947-1952

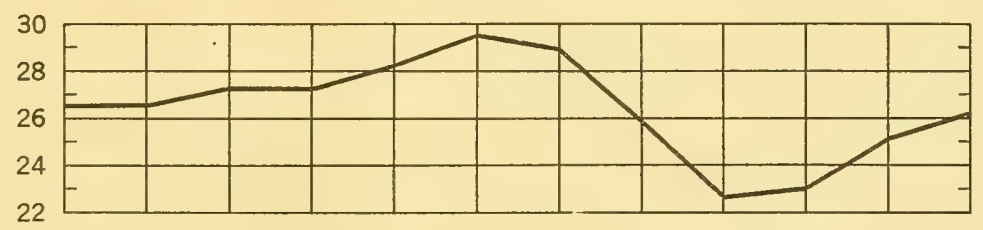

CEDAR KEYS, FLA. 1922-1926, 1944-1951

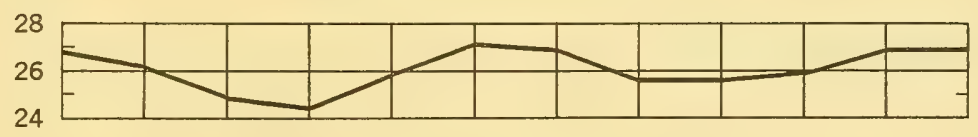

PENSACOLA, FLA. 1924-1952

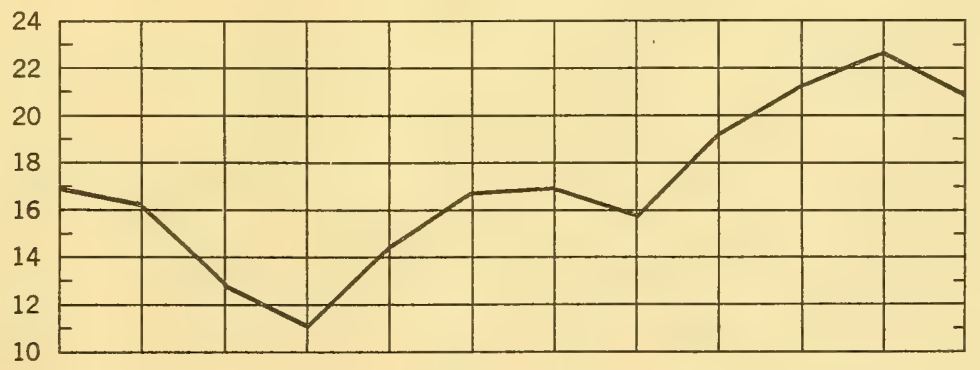

EUGENE ISLAND, LA. 1944-1952

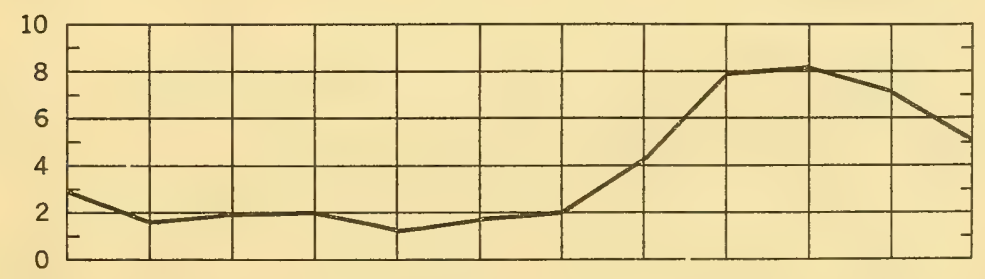


GALVESTON, TEX.

1922-1952

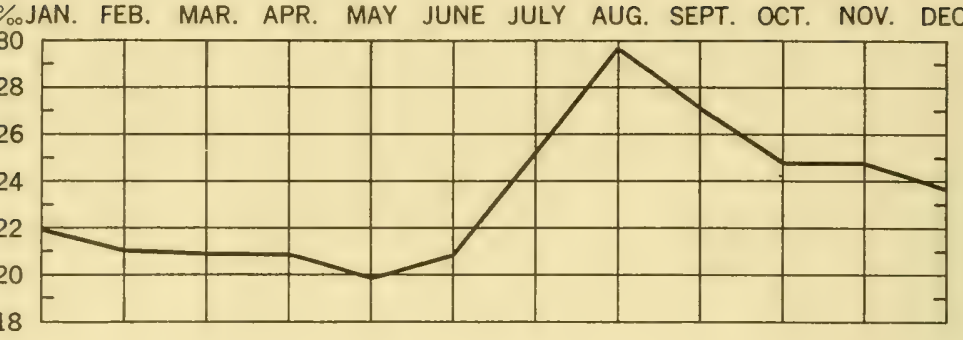

ROCKPORT, TEX.

1948-1952

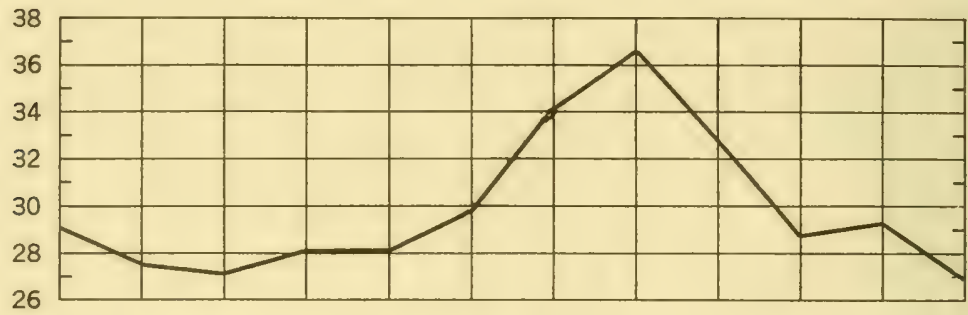

PORT ISABEL, TEX.

1944-1952

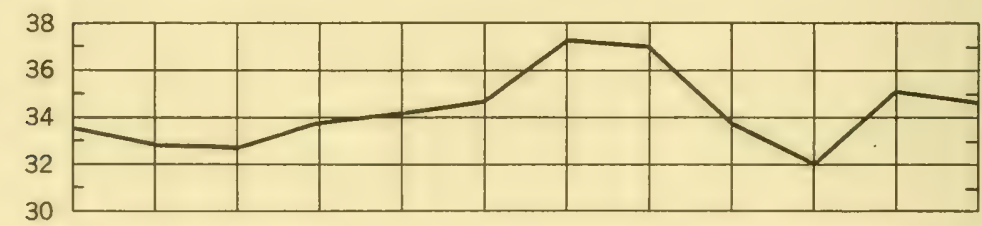

COATZACOALCOS

(Puerto Mexico), MEX.

1946-1952

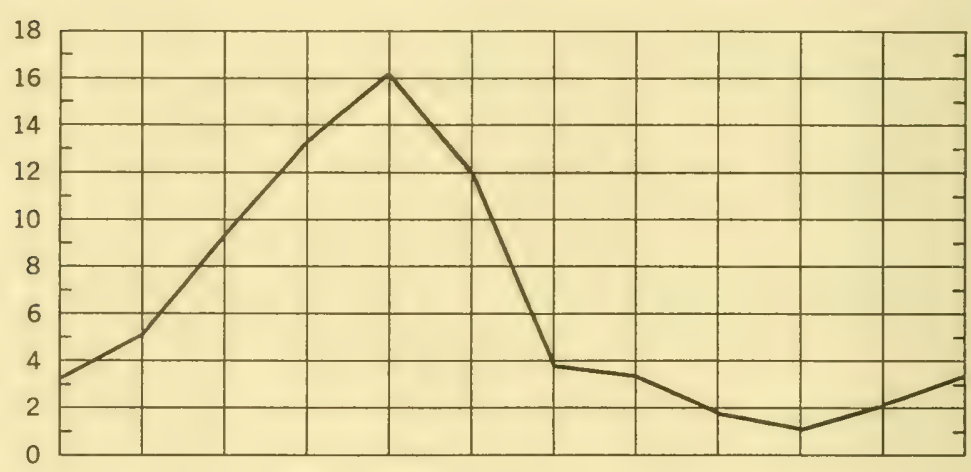

PROGRESO, MEX.

1946-1952

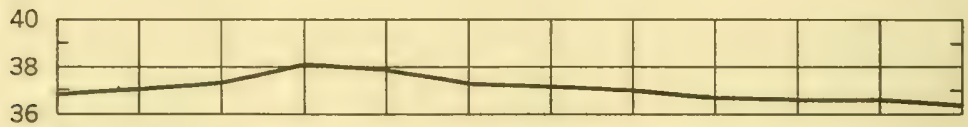

HABANA, CUBA

1947-1952

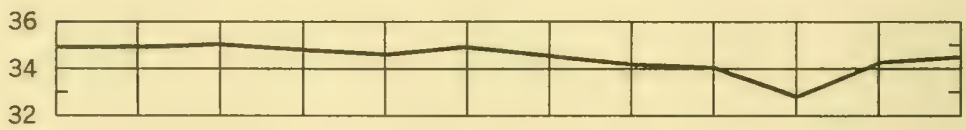

GUANTÁNAMO BAY, CUBA 1947-1952

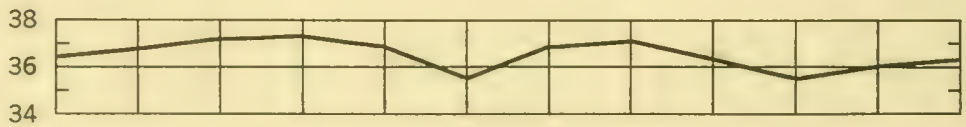


Table 2. Corresponding Densities and Salinities

(Density at $15^{\circ} \mathrm{C}$. -- Salinity in parts per 1000)

\begin{tabular}{|c|c|c|c|c|c|c|c|c|c|c|c|}
\hline $\begin{array}{l}\text { Den- } \\
\text { sity }\end{array}$ & $\begin{array}{c}\text { Sal- } \\
\text { inity }\end{array}$ & $\begin{array}{l}\text { Den- } \\
\text { sity }\end{array}$ & $\begin{array}{l}\text { Sal- } \\
\text { inity }\end{array}$ & $\begin{array}{l}\text { Den- } \\
\text { sity }\end{array}$ & $\begin{array}{l}\text { Sal- } \\
\text { inity }\end{array}$ & $\begin{array}{l}\text { Den- } \\
\text { sity }\end{array}$ & $\begin{array}{l}\text { Sal- } \\
\text { inity }\end{array}$ & $\begin{array}{l}\text { Den- } \\
\text { sity }\end{array}$ & $\begin{array}{l}\text { Sal- } \\
\text { inity }\end{array}$ & $\begin{array}{l}\text { Den- } \\
\text { sity }\end{array}$ & $\begin{array}{l}\text { Sal- } \\
\text { inity }\end{array}$ \\
\hline 0.9991 & 0.0 & 1.0046 & 7.1 & 1.0101 & 14.2 & 1.0156 & 21.4 & 1.0211 & 28.6 & 1.0266 & 35.8 \\
\hline 0.9992 & 0.0 & 1.0047 & 7.2 & 1.0102 & 14.4 & 1.0157 & 21.6 & 1.0212 & 28.8 & 1.0267 & 35.9 \\
\hline 0.9993 & 0.2 & 1.0048 & 7.3 & 1.0103 & 14.5 & 1.0158 & 21.7 & 1.0213 & 28.9 & 1.0268 & 36.0 \\
\hline 0.9994 & 0.3 & 1.0049 & 7.5 & 1.0104 & 14.6 & 1.0159 & 21.8 & 1.0214 & 29.0 & 1.0269 & 36.2 \\
\hline 0.9995 & 0.4 & 1.0050 & 7.6 & 1.0105 & 14.8 & 1.0160 & 22.0 & 1.0215 & 29.1 & 1.0270 & 36.3 \\
\hline 0.9996 & 0.6 & 1.0051 & 7.7 & 1.0106 & 14.9 & 1.0161 & 22.1 & 1.0216 & 29.3 & 1.0271 & 36.4 \\
\hline & 0.7 & 1.0052 & 7.9 & 1.0107 & 15.0 & 1.0162 & 22.2 & 1.0217 & 29.4 & 1.0272 & 36.6 \\
\hline 0.9998 & 0.8 & 1.0053 & 8.0 & 1.0108 & 15.2 & 1.0163 & 22.4 & 1.0218 & 29.5 & 1.0273 & 36.7 \\
\hline 0.9999 & 0.9 & & 8.1 & 1.0109 & 15.3 & 1.0164 & 22.5 & 1.0219 & 29.7 & 1.0274 & 36.8 \\
\hline 1.0000 & 1.1 & 1.0055 & 8.2 & 1.0110 & 15.4 & 1.0165 & 22.6 & 1.0220 & 29.8 & 1.0275 & 37.0 \\
\hline 1.0001 & 1.2 & 1.0056 & 8.4 & 1.0111 & 15.6 & 1.0166 & 22.7 & 1.0221 & 29.9 & . 0276 & 37.1 \\
\hline 1.0002 & 1.3 & 1.0057 & 8.5 & 1.0112 & 15.7 & 1.0167 & 22.9 & 1.0222 & 30.1 & .0277 & 37.2 \\
\hline 1.0003 & 1.5 & 1.0058 & 8.6 & 1.0113 & 15.8 & 1.0168 & 23.0 & 1.0223 & 30.2 & 1.0278 & 37.3 \\
\hline 1.0004 & 1.6 & 1.0059 & 8.8 & 1.0114 & 16.0 & 1.0169 & 23.1 & 1.0224 & 30.3 & 1.0279 & 37.5 \\
\hline 1.0005 & 1.7 & 1.0060 & 8.9 & 1.0115 & 16.1 & 1.0170 & 23.3 & 1.0225 & 30.4 & 1.0280 & 37.6 \\
\hline 1.0006 & 1.9 & 1.0061 & 9.0 & 1.0116 & 16.2 & 1.0171 & 23.4 & 1.0226 & 30.6 & .0281 & 37.7 \\
\hline 1.0007 & 2.0 & 1.0062 & 9.2 & 1.0117 & 16.3 & 1.0172 & 23.5 & 227 & 30.7 & 282 & 37.9 \\
\hline 1.0008 & 2.1 & 1.0063 & 9.3 & 1.0118 & 16.5 & 1.0173 & 23.7 & 1.0228 & 30.8 & 1.0283 & 38.0 \\
\hline 1.0009 & 2.2 & 1.0064 & 9.4 & 1.0119 & 16.6 & 1.0174 & 23.8 & 229 & 31.0 & 1.0284 & 38.1 \\
\hline 1.0010 & 2.4 & 1.0065 & 9.6 & 1.0120 & 16.7 & 1.0175 & 23.9 & 1.0230 & 31.1 & 1.0285 & 38.2 \\
\hline 1.0011 & 2.5 & 1.0066 & 9.7 & & & & 24.1 & & 31.2 & & 38.4 \\
\hline 1.0012 & 2.6 & 1.0067 & 9.8 & 1.0122 & 17.0 & 1.0 & 24.2 & & 31.4 & & 38.5 \\
\hline 1.0013 & 2.8 & 1.0068 & 9.9 & 1.0123 & 17.1 & 1.0178 & 24.3 & 1.0233 & 31.5 & 1.0288 & 38.6 \\
\hline 1.0014 & 2.9 & 1.0069 & 10.1 & 1.0124 & 17.3 & 1.0179 & 24.4 & 1.0234 & 31.6 & 1.0289 & 38.8 \\
\hline 1.0015 & 3.0 & 1.0070 & 10.2 & 1.0125 & 17.4 & 1.0180 & 24.6 & 1.0235 & 31.8 & 1.0290 & 38.9 \\
\hline 1.0016 & 3.2 & 0071 & 10.3 & 1.0126 & 17.5 & 1.0181 & 24.7 & .0236 & 31.9 & .0291 & 39.0 \\
\hline 1.0017 & 3.3 & 1.0072 & .5 & 1.0127 & 17.7 & 1.0182 & 24.8 & 1.0237 & 32.0 & 1.0292 & 39.2 \\
\hline 1.0018 & 3.4 & 1.0073 & 10.6 & 1.0128 & 17.8 & 1.0183 & 25.0 & 1.0238 & 32.1 & . 0293 & 39.3 \\
\hline 1.0019 & 3.5 & 1.0074 & 10.7 & 1.0129 & 17.9 & 1.0184 & 25.1 & 1.0239 & 32.3 & 1.0294 & 39.4 \\
\hline 1.0020 & 3.7 & 1.0075 & 10.8 & 1.0130 & 18.0 & 1.0185 & 25.2 & 1.0240 & 32.4 & 1.0295 & 39.6 \\
\hline 1.0021 & 3.8 & & & & & & 25.4 & & 32.5 & & 39.7 \\
\hline & & 1.00 & & 1.0132 & & & & & 32.7 & & 39.8 \\
\hline 1.0023 & 4.1 & 1.0078 & 11.2 & 1.0133 & 18.4 & 1.0188 & 25.6 & 1.0243 & 32.8 & 1.0298 & 39.9 \\
\hline 1.0024 & 4. & 1.0079 & 11.4 & & 18.6 & & 25.8 & 1.0244 & 32.9 . & 1.0299 & 40.1 \\
\hline 1.0025 & 4.3 & 1.0080 & 11.5 & 1.0135 & 18.7 & 1.0190 & 25.9 & 1.0245 & 33.1 & 1.0300 & 40.2 \\
\hline & & & & & & & & & 33.2 & & \\
\hline 1.0027 & & & & & .0 & & 26.1 & & 33.3 & & 40.4 \\
\hline 1.0028 & & 1.008 & 11.9 & 1.0 & 19.1 & 193 & 26.3 & & 33.5 & .0303 & 40.6 \\
\hline 1.0029 & 4.8 & 1.0084 & 12.0 & 1.0139 & 19.2 & 1.0194 & 26.4 & 1.0249 & 33.6 & 1.0304 & 40.7 \\
\hline 1.0030 & 5.0 & 1.0085 & 12.2 & 1.0140 & 19.3 & 1.0195 & 26.5 & 1.0250 & 33.7 & 1.0305 & 40.8 \\
\hline 1.00 & & & & & 5 & & & & 33.8 & & \\
\hline & & & & 0142 & 19 & & 26 & & 34.0 & 307 & 41.1 \\
\hline 1.0033 & & & & & & & & & & & 41.2 \\
\hline 1.0034 & 5. & 1.0089 & 12.7 & 1.0144 & 19.9 & 1.0199 & 27.1 & 1.0254 & 34.2 & 1.0309 & 41.4 \\
\hline 1.0035 & 5.6 & 1.0090 & 12.8 & 1.0145 & 20.0 & 1.0200 & 27.2 & 1.0255 & 34.4 & 1.0310 & 41.5 \\
\hline & & & & & & & & & 34.5 & & \\
\hline & & & & & & & 27 & & 34.6 & 312 & 41.7 \\
\hline & & & & & & & & & 34.8 & 1.0313 & 41.9 \\
\hline 1.0039 & $6:$ & 1.0094 & 13.3 & 1.0149 & 20.5 & 1.0 & 27.7 & 259 & 34.9 & 1.0314 & 42.0 \\
\hline 1.0040 & 6.3 & 1.0095 & 13.5 & 1.0150 & 20.6 & 1.0205 & 27.8 & 1.0260 & 35.0 & 1.0315 & 42.1 \\
\hline & & & & & & & 28 & & 35.1 & & \\
\hline & & & & & 20 & & 28 & & 35.3 & 17 & 42.4 \\
\hline 1.0043 & 6.7 & 1.0098 & 13. & 1.0153 & 21. & 1.0 & 28.2 & 1.0 & 35.4 & & 42.5 \\
\hline 1.0044 & 6.8 & 1.0099 & 14.0 & 1.0154 & 21.2 & 1.0209 & 28.4 & 1.0264 & 35.5 & 1.0319 & 42.7 \\
\hline 1.0045 & 6.9 & 1.0100 & 14.1 & 1.0155 & 21.3 & 1.0210 & 28.5 & 1.0265 & 35.7 & 1.0320 & 42.8 \\
\hline
\end{tabular}




\section{Sea Water Density at Various Temperatures}

The purpose of this graph is to provide the density of sea water at any temperature apt to be encountered when the density at the standard temperature of $59^{\circ} \mathrm{F}$. $\left(15^{\circ} \mathrm{C}\right.$. $)$ is known. It is intended primarily for use with this density publication which gives densities at $59^{\circ} \mathrm{F}$. and with Coast and Geodetic Survey Special Publication No. 278 which gives sea water temperatures for the same stations.

To convert a density at $59^{\circ} \mathrm{F}$. to density at another temperature, enter the graph horizontally from the left with the known density and downward from the top with the desired temperature; the position of the point of intersection with respect to the curves gives the density at the desired temperature. Interpolate between curves when necessary. For example, by this method, water having a density of 1.0162 at $59^{\circ} \mathrm{F}$. is found to have a density of 1.0124 at $85^{\circ} \mathrm{F}$.

The densities are referred to the density of fresh water at $4^{\circ} \mathrm{C} .\left(39.2^{\circ} \mathrm{F}\right.$.) as unity.

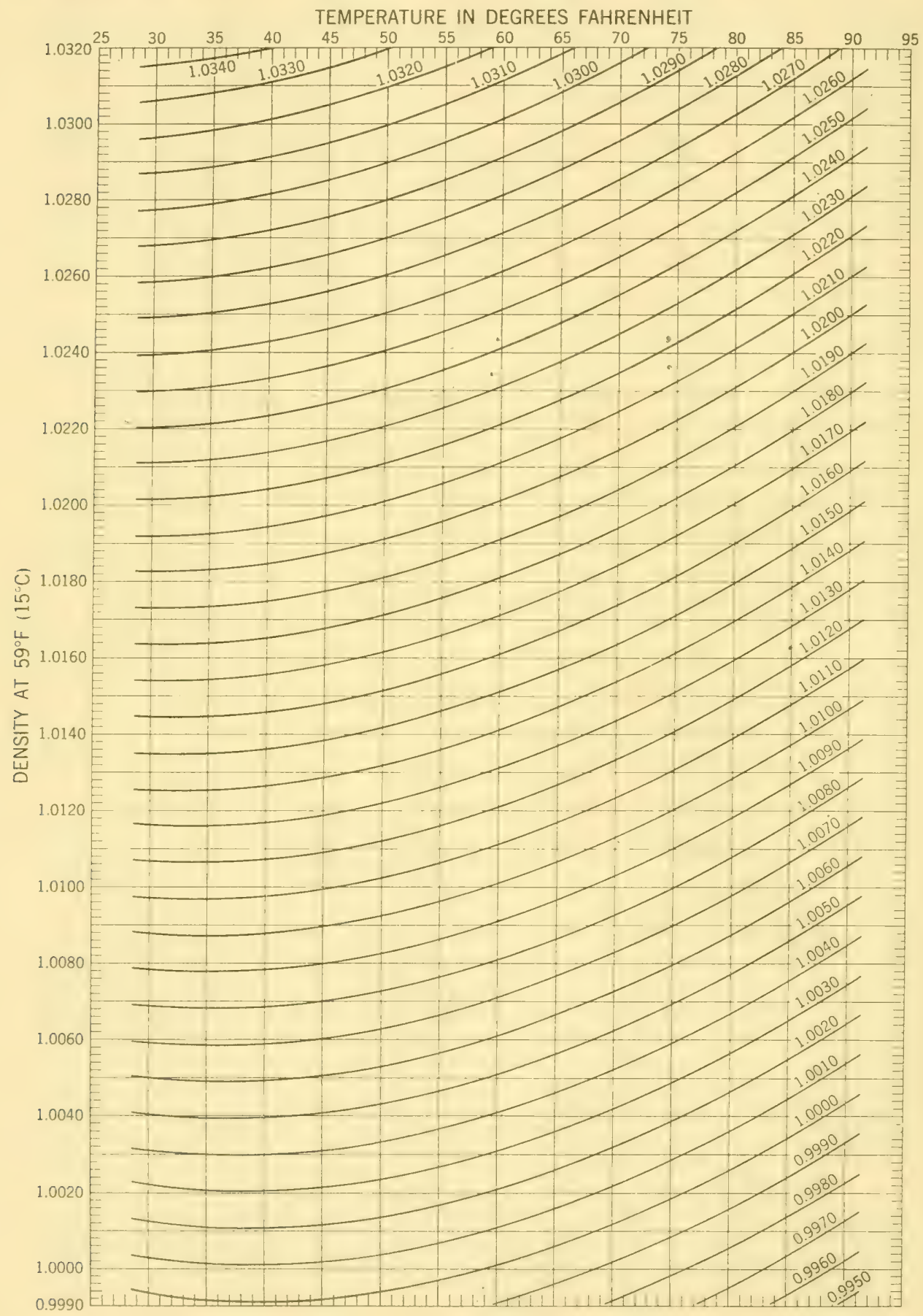






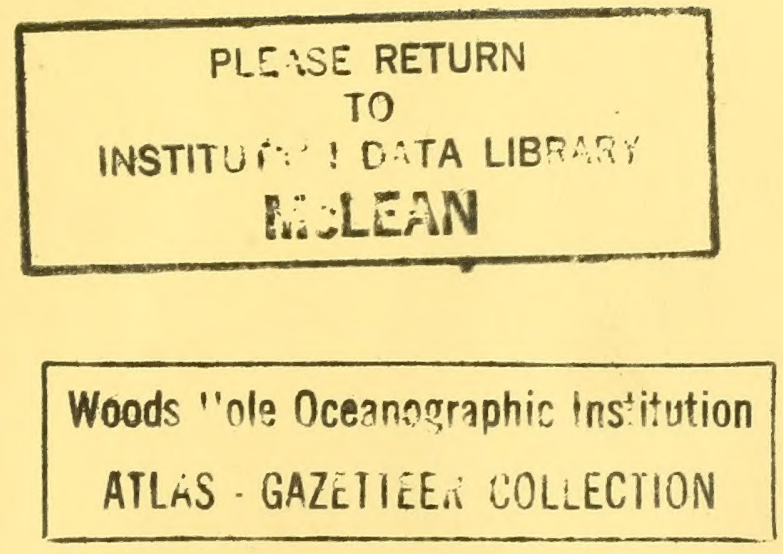


